\title{
Cycle-related solar vacuum ultraviolet (VUV) variability
}

\author{
Dissertation \\ zur Erlangung des mathematisch-naturwissenschaftlichen Doktorgrades \\ "Doctor rerum naturalium" \\ der Georg-August-Universität Göttingen \\ im Promotionsprogramm PROPHYS \\ der Georg-August University School of Science (GAUSS)
}

vorgelegt von

Farhad Shakeri

aus Tehran, Iran

Göttingen, 2014 
Betreuungsausschuss

Prof. Dr. Stefan Dreizler

Institut für Astrophysik, Georg-August-Universität Göttingen, Germany

Prof. Dr. Sami K. Solanki

Max-Planck-Institut für Sonnensystemforschung, Göttingen, Germany

Dr. Luca Teriaca

Max-Planck-Institut für Sonnensystemforschung, Göttingen, Germany

Mitglieder der Prüfungskommision

Referent: Prof. Dr. Stefan Dreizler

Institut für Astrophysik, Georg-August-Universität Göttingen, Germany

Korreferent: Prof. Dr. Sami K. Solanki

Max-Planck-Institut für Sonnensystemforschung, Göttingen, Germany

Weitere Mitglieder der Prüfungskommission:

Prof. Dr. Jörg Büchner

Max-Planck-Institut für Sonnensystemforschung, Göttingen, Germany

Prof. Dr. Laurent Gizon

Institut für Astrophysik, Georg-August-Universität Göttingen, Germany

Prof. Dr. Wolfgang Glatzel

Institut für Astrophysik, Georg-August-Universität Göttingen, Germany

Prof. Dr. Hardi Peter

Max-Planck-Institut für Sonnensystemforschung, Göttingen, Germany

Tag der mündlichen Prüfung: 20.01.2015 


\section{Bibliografische Information der Deutschen Nationalbibliothek}

Die Deutsche Nationalbibliothek verzeichnet diese Publikation in der Deutschen Nationalbibliografie; detaillierte bibliografische Daten sind im Internet über http: //dnb . d-nb . de abrufbar.

ISBN 978-3-944072-10-4

uni-edition $\mathrm{GmbH} 2015$

http://www.uni-edition.de

(C) Farhad Shakeri

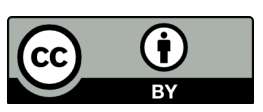

This work is distributed under a

Creative Commons Attribution 3.0 License

Printed in Germany 



\section{Contents}

$\begin{array}{lr}\text { Summary } & 7\end{array}$

1 Introduction 9

1.1 Structure of the Solar atmosphere . . . . . . . . . . . . . . . . 10

1.2 Solar Vacuum UltraViolet (VUV) radiation . . . . . . . . . . . . . . 12

1.3 Solar magnetism . . . . . . . . . . . . . . . . 13

1.3.1 Quiet Sun magnetic field . . . . . . . . . . . . . . 15

1.3.1.1 Local dynamo . . . . . . . . . . . . . . 16

1.3.2 Magnetic field in the solar atmosphere and the network pattern . . 16

1.4 Heating mechanisms . . . . . . . . . . . . . . . . 18

1.4.1 Nanoflare model . . . . . . . . . . . . . . . . . 18

1.4.1.1 Heating of the transition region . . . . . . . . . 19

1.4.2 Acoustic waves and chromospheric heating . . . . . . . . . . 20

1.5 Radiance distribution of the Quiet Sun . . . . . . . . . . . . . . 22

1.5.1 Lognormal distribution . . . . . . . . . . . . . . 23

1.611 -year sunspot cycle . . . . . . . . . . . . . . . 25

1.6.1 Anomalous solar cycle $23 \ldots \ldots 26$

2 Instrumentation $\quad \mathbf{2 9}$

2.1 Coronal Diagnostic Spectrometer (CDS) . . . . . . . . . . . . . . . 29

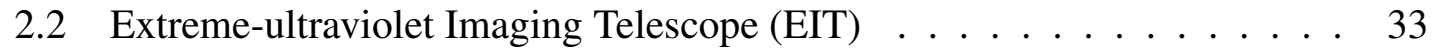

3 Observations and Data reduction $\quad 37$

3.1 Observational data . . . . . . . . . . . . . . . . . . 37

$3.2 \mathrm{CDS} / \mathrm{NIS}$ data structure $\ldots \ldots \ldots \ldots \ldots$

3.3 Data reduction . . . . . . . . . . . . . . . 38

3.3.1 Bias subtraction . . . . . . . . . . . . . 38

3.3.2 Removing cosmic ray hits . . . . . . . . . . . . 38

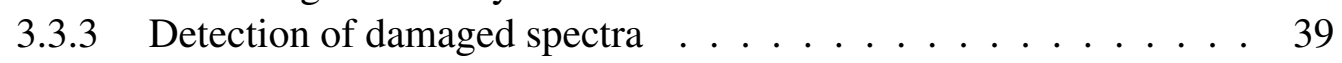

3.3.4 Image distortion and other effects ... . . . . . . . . . 41

3.3.5 Data calibration . . . . . . . . . . . . . . . 42

3.3.6 Least-square fitting of the spectra . . . . . . . . . . . . . 43

3.3 .7 Noise propagation ...................... 45

3.3.7.1 Photon noise . . . . . . . . . . . . 46

3.3.7.2 Detector noise . . . . . . . . . . . . 46

3.3.7.3 Total noise . . . . . . . . . . . . . 47 
3.3.7.4 Error in the intensity . . . . . . . . . . . . . . 47

3.4 CDS point-spread function and data binning . . . . . . . . . . . . . 47

3.5 Data exclusion . . . . . . . . . . . . . . . . 48

4 Analyses and results $\quad \mathbf{5 1}$

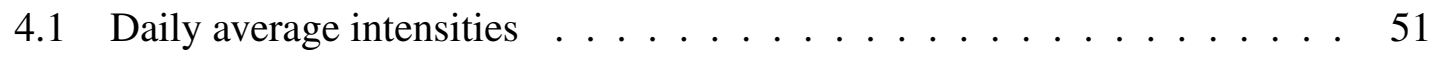

4.2 Contrast ratios . . . . . . . . . . . . . . . . . . 54

4.2.1 Peculiar case of binsize 1 . . . . . . . . . . . 57

4.2.2 Effect on CRs of spectral broadening after SOHO recovery . . . 60

4.2.3 Co-spatiality between $\mathrm{O} v$ and $\mathrm{He}_{\mathrm{I}} \ldots \ldots$. . . . . . . . . . 62

4.3 Intensity distribution of the quiet Sun radiation and lognormal fitting . . . 64

4.4 Data exclusion based on $\chi^{2}$ of the lognormal fits . . . . . . . . . . . . . . 68

4.5 Network thickness . . . . . . . . . . . . . . . . . 70

4.6 Periodic appearance of AR in minimum $22 \ldots \ldots . \ldots 75$

5 Discussion and conclusion $\quad 81$

$\begin{array}{llr}6 & \text { Outlook } & 85\end{array}$

$\begin{array}{lr}\text { Bibliography } & 89\end{array}$

$\begin{array}{ll}\text { Acknowledgements } & 105\end{array}$ 


\section{Summary}

The Sun is the most important source of energy to Earth and the main external driver of the climate system. Although it seems pretty much constant when being looked at in daily life, the activity of the Sun is in fact a variable phenomenon on all time scales. Since its discovery in mid nineteenth century, the roughly 11-year solar activity cycle, as the main quasi-periodic variability feature of the Sun, has been continuously monitored and analysed. The importance of investigating the solar variability comes from the fact that it has direct impact on the Earth's atmosphere. While, for instance, the Total Solar Irradiance (TSI, alternatively called the solar constant) determines the average temperature of the Earth's atmosphere, the UV radiation affects the photochemistry of the Earth's atmosphere. The vacuum ultraviolet (VUV) spectrum, as a portion of the UV radiation with wavelengths less than $200 \mathrm{~nm}$, is the main source of energy for the Earth's middle and upper atmosphere and controls many aeronomic processes.

Depending on the wavelength, the VUV radiation originates from different heights and structures in the solar atmosphere, from the relatively cold (about $4000 \mathrm{~K}$ ) temperature minimum at the top of the photosphere (mostly generating continuum emission at the long wavelength end) to the million Kelvin coronal structures that generate the forest of spectral lines that crowds the extreme ultraviolet (EUV) at the short wavelength range (from $120 \mathrm{~nm}$ down to $10 \mathrm{~nm}$ ). In this respect, investigating the VUV radiation, besides its significance in understanding the processes in the Earth's high-altitude atmosphere, provides us crucial information about the physical processes in the solar atmosphere. Despite its importance, the records of VUV measurements is limited to the space age, since the radiation in this wavelength range is absorbed in the Earth's atmosphere and its measurement is possible only through satellite-based observations.

Various studies has shown that the Sun was in an extremely inactive state during the prolonged minimum of the cycle 23 compared to other recent minima. Following these studies, in this thesis work, it was tried to have a closer look at the variability of the quiet Sun network and internetwork radiance between the last two solar minima. The aim of this dissertation is to find out whether there is a change in the radiance properties of the upper chromosphere and transition region (at some specific wavelengths of the VUV spectrum), over the last solar cycle.

We carried out a statistical study of the radiance variability of the chromospheric He I $58.4 \mathrm{~nm}$ (formation temperature $T_{f} \sim 20,000 \mathrm{~K}$ ) and transition region $\mathrm{O}$ v $63.0 \mathrm{~nm}$ $\left(T_{f} \sim 250,000 \mathrm{~K}\right)$ spectral lines in the quiet Sun close to disc-center, over a period of 15 years from 1996 to 2011, with the main focus on comparing the last two solar minima. We used observations from the Coronal Diagnostic Spectrometer (CDS) on board the Solar and Heliospheric Observatory (SOHO), which provides daily observations of the Sun in various wavelengths and has a record long enough to cover the last two solar minima. 
It was found, that the radiance distribution of the transition region $\mathrm{O} v$ line, has undergone remarkable changes during the solar minimum 23 compared to the previous minimum. Such variations were not seen in the radiance distribution of the chromospheric He I line. These results suggest that different heating mechanisms may trigger radiation from the chromosphere and the transition region at the quiet Sun. While the latter seems to be more magnetic in nature, the former is less affected by the solar magnetic activity.

The results from CDS data were later compared to those obtained from the ExtremeUltraviolet Imaging Telescope (EIT), also on board SOHO, by looking at the transition region HeII $30.4 \mathrm{~nm}\left(T_{f} \sim 80,000 \mathrm{~K}\right)$ and coronal/transition region boundary FeIX-X $17.1 \mathrm{~nm}$ $\left(T_{f} \sim 1.3 \times 10^{6} \mathrm{~K}\right)$ spectral lines.

This dissertation consists of 6 chapters. Chapter 1 gives a summarized overview of our current knowledge about the solar VUV variability as a result of the solar magnetic activity cycle. Chapter 2 contains technical information about the instruments whose data have been exploited in this study. The data reduction procedure and the results are explained in Chapters 3 and 4, respectively. The thesis is rounded-up in chapter 5, where the results are summarised and discussed. An outlook to the potential further investigations is provided in Chapter 6. 


\section{Introduction}

The Sun, in the current era of its life, is a G-type main-sequence star. Like for all other stars on the lower main-sequence, the Sun's energy is generated through thermonuclear reactions. In the extremely hot conditions of the core, with a temperature of about $1.5 \times 10^{7} \mathrm{~K}$, the hydrogen nuclei fuse with each other and convert into helium mostly through proton-proton chains, a process known as nuclear fusion. The energy generated in the core is transported to the surface of the Sun, initially through thermal radiation and then by thermal convection. These different energy transport mechanisms divide the Sun interior into a radiation zone (from core up to approximately 0.7 solar radii, $0.7 \mathrm{R}_{\odot}$ ) and convection zone (from 0.7 $\mathrm{R}_{\odot}$ up to the surface). The solar energy is eventually radiated into outer space in the form of electromagnetic radiation, with a spectrum similar to that of a black body with a temperature of $\sim 5780 \mathrm{~K}$ and with a total power of $\sim 4 \times 10^{26} \mathrm{~W}$ (Phillips 1995).

Considering the distance between the Earth and the Sun and the relatively small size of the Earth, only an extremely small fraction of this power reaches the Earth. The solar radiative power at the top of the Earth's atmosphere is approximately $10^{17} \mathrm{~W}$, about a billion times smaller than the solar output power. Nevertheless, in comparison to other sources of energy on Earth, such as cosmic ray particles, geothermal energy and tidal energy, amounting to roughly $10^{10} \mathrm{~W}, 10^{13} \mathrm{~W}$ and $10^{12} \mathrm{~W}$, respectively (Beer et al. 2006), the Sun is by far the main source of energy on Earth.

The radiation escaping from the solar atmosphere contains crucial information about the temperature and dynamics of the plasma above the surface. This radiation includes emission lines from very hot plasma, with temperatures from tens of thousands up to millions of Kelvin. The reason why the plasma temperature in the solar atmosphere increases dramatically with altitude above the visible surface is still not fully understood and is the main topic of solar physics research, but what is commonly accepted is that the magnetic field lines, anchored in the convection zone and being dragged around and twisted by the turbulent motions of plasma in the photosphere, are the main energy sources for maintaining these high temperatures (Walsh and Ireland 2003). Almost all phenomena observed in the solar atmosphere are significantly influenced by the Sun's magnetic field. The solar magnetic field is believed to be generated by the motion of the plasma in a transition layer between the radiation zone and convection zone, called the tachocline (see Sec. 3.2 of Miesch 2005), through a process known as the solar global dynamo (Parker 1955; Ossendrijver 2003). Surface observations have shown that the rotation rate of the Sun varies with latitude, with periods from 35 days at the equator down to 25 days at polar regions. Helioseismology, which studies the interior of the Sun through the properties of the acoustic waves that travel within it, suggests that the differential rotation observed on the solar surface is maintained inside the convection zone (except for a shallow layer right below the surface) down to the tachocline, below which the material in the radiation zone 
rotates homogeneously, similar to a rigid body with a period of 27 days. The solar global dynamo which is a consequences of the turbulent convection, the differential rotation and the solar meridional circulation, play the key roles in the magnetic activity of the Sun, whose most familiar manifestation is the 11-year sunspot cycle.

\subsection{Structure of the Solar atmosphere}

The atmosphere of the Sun is usually described in terms of a multi-layer atmosphere. The hot plasma rising up in the convection zone, reaches a level at which the opacity is low enough for the photons to make a transition from random-walk, within the Sun interior, to escape to outer space. This happens at the solar surface, where by definition the optical depth is close to unity. Usually, the solar surface is defined as the region where the condition of the optical depth being unity at a wavelength of $500 \mathrm{~nm}\left(\tau_{500}=1\right)$ is fulfilled. The escape of the radiation, also known as radiative cooling, causes the hot plasma to cool down, compress, loose its buoyancy and eventually sink back into the convection zone. The horizontal pressure gradient then pushes the surrounding plasma toward the void produced by the plasma downflow. This induced circulation of plasma creates convective cells, known as granules, in a shallow layer of about $100 \mathrm{~km}$ above the solar surface.

As long as low spatial resolution is considered (based on the traditional 1D model (Athay (1976), Vernazza et al. (1981)), the temperature profile of the solar atmosphere is highly structured (see Fig. 1.1). Starting from the solar surface, the temperature decreases from roughly $6300 \mathrm{~K}$ down to $\sim 4000 \mathrm{~K}$ at a height of about $300 \mathrm{~km}$ (temperature minimum height). This shallow layer, characterized by populated granulation cells, with typical size of 1-2 Mm and life-time of few minutes, is the photosphere.

The solar atmosphere above the photosphere is divided into three layers:

Chromosphere: It is the second layer of the solar atmosphere from the bottom. With a highly dynamic structure, it extends from the photosphere up to about $5000 \mathrm{~km}$ above the surface. Despite being relatively thin, the density in the chromosphere drops rapidly from $2 \times 10^{-4} \mathrm{~kg} / \mathrm{m}^{3}$ at its boundary with the photosphere to as low as $1.6 \times 10^{-11} \mathrm{~kg} / \mathrm{m}^{3}$, where it merges into the overlying transition region (Avrett and Loeser (1992), Kontar et al. (2008)). The temperature in the chromosphere increases from temperature minimum $\sim 4000 \mathrm{~K}$ to as high as $\sim 2.5 \times 10^{4} \mathrm{~K}$. At these high temperatures, while many of the species are already ionized, hydrogen atoms are still abundant in their neutral state. Therefore, the thermal emission in this layer is either absorbed or scattered by neutral hydrogen. This, plus the extremely low density of the chromosphere (with respect to the photosphere), makes the chromosphere almost invisible against the bright underlying photosphere. Note that, only a very small portion of Sun's luminosity, about $0.01 \%$, originates from the chromosphere (over the quiet regions) (Avrett 1981).

Transition region: It is a thin layer (about $200 \mathrm{~km}$ in 1-D classical models) between the chromosphere and the corona, where the temperature increases abruptly up to approximately $10^{6} \mathrm{~K}$ and the density drops dramatically to $\sim 10^{-13} \mathrm{~kg} / \mathrm{m}^{3}$. In reality, the transition region (TR) is not a layer with well-determined boundaries. The reason is that, from the solar surface to the upper chromosphere, it is mainly the gravity that shapes the 


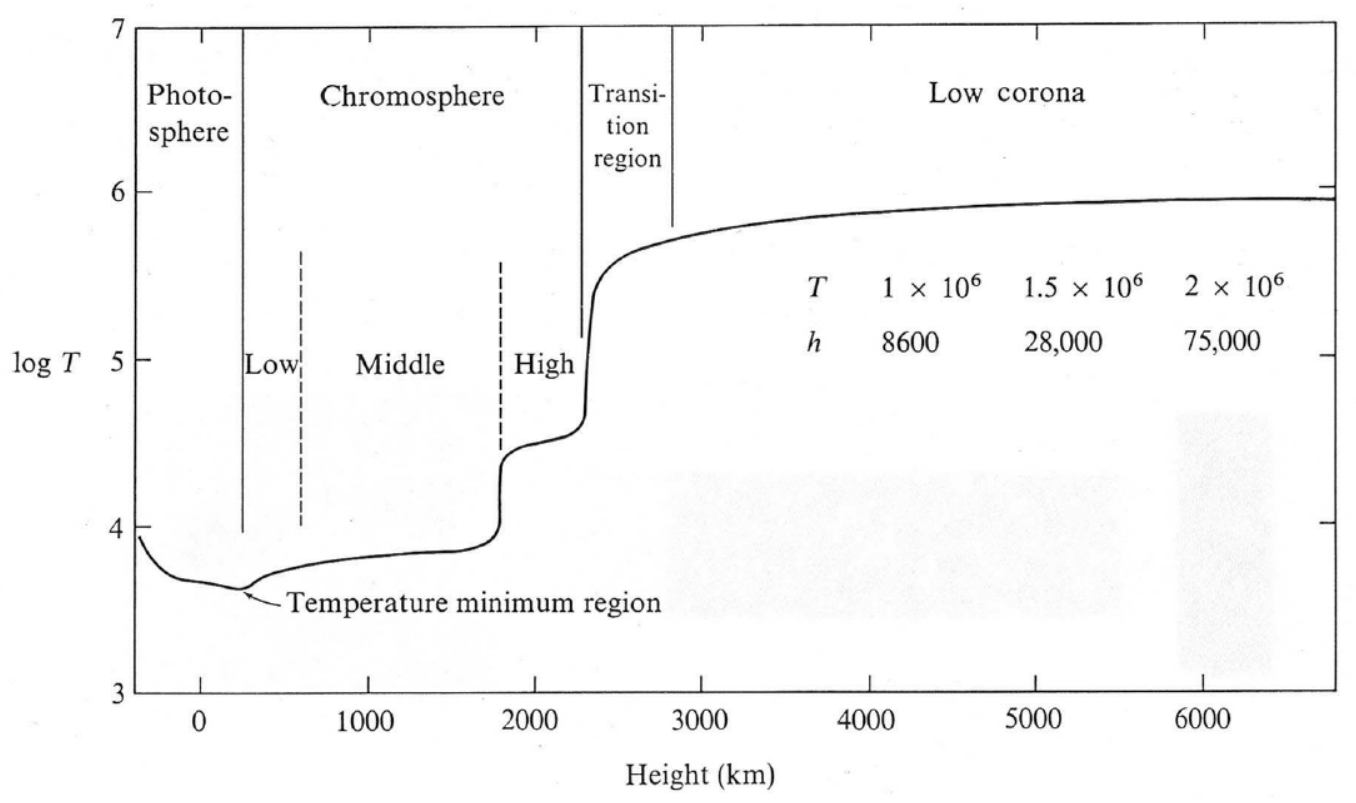

Figure 1.1: 1D temperature gradient in the solar atmosphere (figure taken from Athay 1976).

atmosphere and gives it a layered structure (with roughly horizontally extended features like sunspots on the photosphere). In the transition region, as well as in the overlying corona, the extremely dynamic magnetic forces dominate over the gravity and play the main role in the generation of most observable features. As in the chromosphere, the majority of TR radiation are emission lines from the VUV spectral range, with a tendency toward the FUV part of the solar spectrum.

Corona: This is the outermost layer of the solar atmosphere. Extending millions of kilometres from TR to outer space, the temperature of the coronal plasma rises up to a few million Kelvin and the density drops to as low as $10^{-12}$ of that of the photosphere (Avrett and Loeser 1992). The extremely low density of the corona results in very low contribution of the coronal brightness to the total brightness of the Sun (almost $10^{-6}$ ), so that it is only visible if the sunlight from the solar disc is blocked. The radiation from the corona has different sources: scattering of the photospheric radiation by free electrons and dust particles, which categorize the corona into K-corona and F-corona, respectively, and emission lines produced by ions, known as E-corona. The corona is the source of large streams of plasma (known as solar wind) and energetic particles.

Note that the traditional 1D atmospheric model by Athay (1976), shown in Fig. 1.1, does not provide a complete picture of the extremely dynamic and inhomogeneous solar atmosphere, since many of the atmospheric phenomena and processes extend over more than one layer (see Wilhelm et al. 2007). Nevertheless, such a simplified picture brings about the question, why does the temperature rise with height? This is indeed contrary to our expectations from thermodynamics. The different heating mechanisms which are thought to be responsible for such high temperatures in the higher solar atmosphere are discussed in Sec. 1.4. 


\subsection{Solar Vacuum UltraViolet (VUV) radiation}

The vacuum ultraviolet (VUV) radiation, as a portion of the UV spectrum with wavelengths between 10 and $200 \mathrm{~nm}$, is the main source of energy for the aeronomic processes in the Earth's upper atmosphere. The VUV/Soft-X radiation (down to $0.5-1 \mathrm{~nm}$ ), contribute in the formation of the Earth's ionosphere by photoionizing the neutral constituents at the top of the atmosphere (Woods and Rottman 2002). Almost all incoming solar VUV radiation is absorbed in the Earth's atmosphere, causing its detection and measurement to be possible only with satellite-based instruments and sounding rockets that are operating above the Earth's atmosphere. A comprehensive review of the record of the VUV observations has been given by Wilhelm et al. (2004).

The VUV radiation is produced by electron's transitions of atoms and ions in the solar atmosphere through ionization and excitation processes. The VUV spectrum originates from different altitudes/structures, covering a variety of temperatures from a minimum of about $4000 \mathrm{~K}$ at the top of the photosphere (corresponding to photospheric continuum radiation) up to more than a few million Kelvin (corresponding to coronal emission lines) (Woods 2002). Fig. 1.2, taken from Woods and Rottman (2002), illustrates the solar irradiance in the VUV wavelength range. The solid black line shows the measured spectrum and the dashed lines give identifications of a few examples of emission lines originating in different layers of the solar atmosphere. The VUV irradiance of the upper photosphere decreases almost by two orders of magnitude from $200 \mathrm{~nm}$ down to $160 \mathrm{~nm}$. The continuum radiation close to $160 \mathrm{~nm}$ is thought to originate from the cool plasma regions at the top of the photosphere (Vernazza et al. 1981), with a brightness temperature close to $4500 \mathrm{~K}$ (Wilhelm et al. 1999; Samain et al. 1975). The decrease in the irradiance of the photospheric VUV radiation is accompanied by the emerging of the chromospheric and transition region emission lines and continua and, at shorter wavelengths, by appearance of coronal lines from heavier species. Of particular interest in the context of this work are the chromospheric $\mathrm{He}$ I $58.4 \mathrm{~nm}$ and transition region $\mathrm{O}$ v $63.0 \mathrm{~nm}$ and $\mathrm{He}$ II $30.4 \mathrm{~nm}$ emission lines. The $\mathrm{O} v$ lines, in general, are emitted from the regions with electron temperatures of $1.6-4 \times 10^{5} \mathrm{~K}$ (Malinovsky 1975). Such a high temperature is produced through complex heating mechanisms in the solar higher atmosphere. Several scenarios have been proposed to explain the strength of transition region emissions: downflow of the material from hotter corona (Gabriel 1976; Athay 1990), existence of cool loops in the lower transition region (Dowdy et al. 1986) and thermal conduction across the magnetic field lines (Rabin and Moore 1984) are some of the proposed scenarios.

The generation of the helium emission lines in the chromosphere and lower transition region is also a matter of debate. Zirin (1988) suggests that helium spectral lines are generated through photoionization-recombination mechanism induced by coronal EUV radiation. This explanation was questioned by Ravindra and Venkatakrishnan (2003), who found that the radiance of the He II $30.4 \mathrm{~nm}$ emission line over the network area is independent of coronal radiation and instead is highly correlated with the magnetic field of the photosphere. By studying the observations from the Coronal Diagnostic Spectrometer (CDS, see Sec. 2.1) and the Extreme-ultraviolet Imaging Telescope (EIT, see Sec. 2.2), Andretta et al. (2003) also came to a similar conclusion, declaring that the formation of the He II $30.4 \mathrm{~nm}$, cannot be explained only by photoionization-recombination mechanism, but also have a collisional component. Finally, Athay (1988) and Seely and Feldman (1985) 


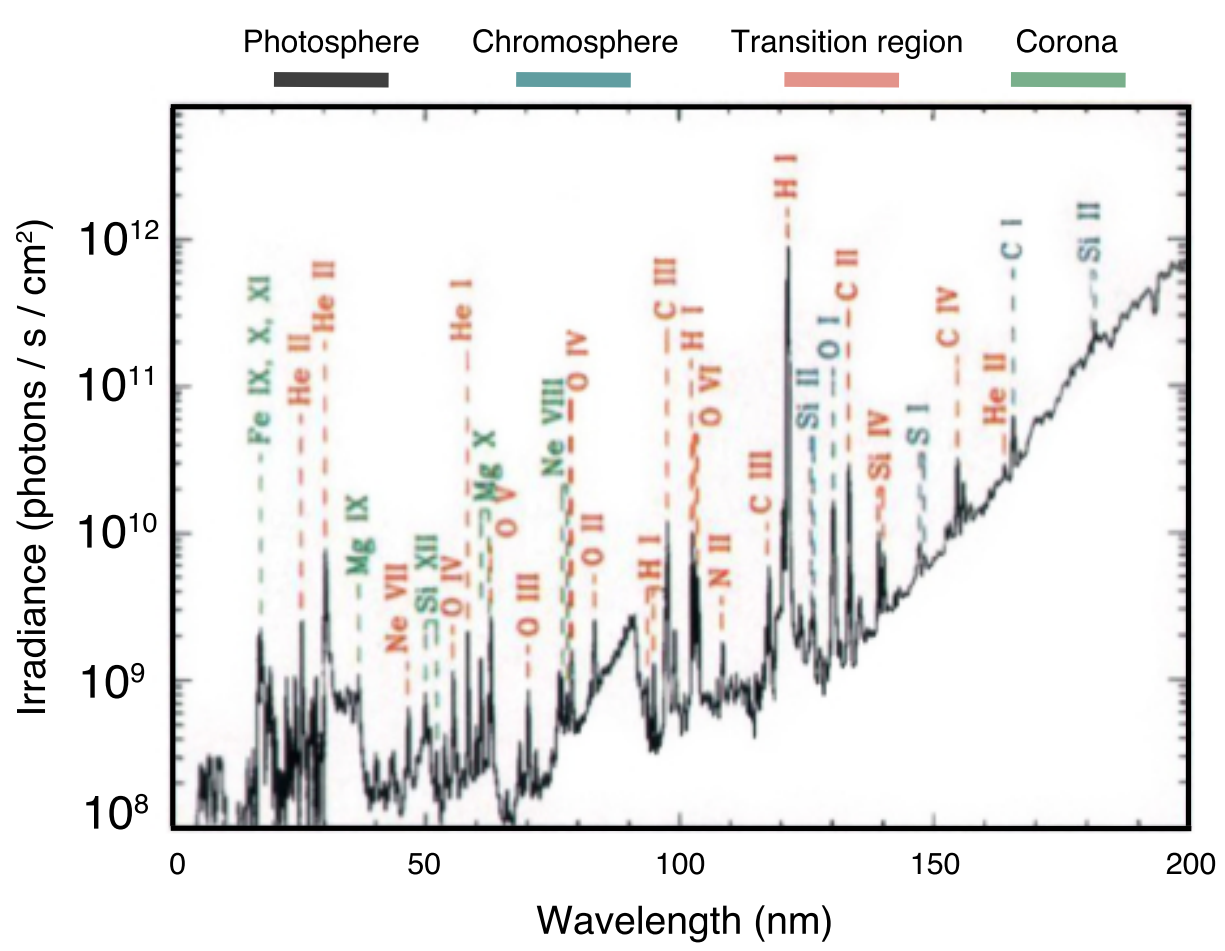

Figure 1.2: Solar VUV irradiance with examples of emission lines from different layers of the solar atmosphere (figure adopted from Woods and Rottman 2002).

discussed the role of collisional excitation in the generation of the helium spectral lines.

\subsection{Solar magnetism}

The solar magnetic field is extremely complex. From the smallest currently observable magnetic features on the solar surface to the global dipole of the Sun, the solar magnetic pattern ranges over a factor of $10^{4}$. The scale in the life-time and evolution of this pattern is even larger, varying by a factor of $10^{6}$, from minute-long evolution of the magnetic fields at the intergranular regions to the decade-long sunspot cycle (Schrijver and Siscoe 2010).

In a model proposed by Parker (1955) dynamo action was suggested to be responsible for the generation of the magnetic field in the convection zone. According to this model, the poloidal magnetic field lines are frozen in the highly ionized plasma in the convection zone. The differential rotation of the Sun twists the poloidal field and produces a toroidal component ( $\omega$ effect). On the other hand, the vertical convective motions of the plasma, being twisted by Corriolis force, produces magnetic loops in the convection zone which can merge together and regenerate and amplify the original poloidal field ( $\alpha$ effect).

Parker (1975) and Schüssler (1979) argued that the dynamo cannot be generated and stored in the convection zone, because the strong toroidal magnetic fields are pushed up toward the surface by magnetic buoyancy relatively fast (compare to the time-scale of the sunspot cycle). They instead suggested that the dynamo is located at the bottom of the convection zone, the idea that later was supported by Schou et al. (1992), who revealed the existence of a shallow layer with high velocity sheer between the convention zone and the 
radiation zone.

Very successful models of the dynamo were developed by Babcock (1961) and later by Leighton (1964). The main concept behind these models is the advection of the poloidal magnetic field by meridional circulation, and shearing of magnetic field lines by differential rotation in the convection zone (Dwivedi and Narain 2008). These models could explain many observational features such as migration of the sunspots toward the poles and sunspot cycle. Using Babcock-Leighton mechanismCharbonneau 2005 and Dikpati and Gilman 2006 predicted strong magnetic field at the poles, which contradicts the observations (see the review by Dikpati et al. 2006). However, this was questioned by Choudhuri et al. (2007) and Jiang et al. (2007), who showed that polar magnetic field cannot be as strong as what was already predicted by the other two studies. Although there have been impressive progress in dynamo models during recent years, yet the exact nature and origin of the solar dynamo is a matter of discussion.

According to Alfvén's theorem, in a perfectly conducting fluid (MHD approximation) such as solar plasma, the magnetic field lines are frozen into the fluid and move with it. The temporal evolution of the magnetic field inside a conducting fluid is controlled by the induction equation:

$$
\frac{\partial \vec{B}}{\partial t}=\nabla \times(\vec{v} \times \vec{B})+\eta \nabla^{2} \vec{B}
$$

where $\eta$ is magnetic diffusivity, $\vec{B}$ is the magnetic field and $\vec{v}$ is the velocity of the fluid material. The first and second terms on the right side of the equation control the motion of the fluid perpendicular to the magnetic field and the diffusivity of the magnetic field, respectively. The ratio between the first and second terms, known as magnetic Reynolds number $R_{m}$ is a measure of freezing and/or diffusion of the field. In the MHD approximation, the conductivity is infinite, the diffusivity $\eta$ is negligible and $R_{m} \gg 1$. Therefore, the magnetic field lines can be carried unchanged with the plasma material.

As a result, in the solar photosphere, the horizontal motions in the supergranulation cells (as well as granulation cells), sweep the magnetic field lines to the boundaries between the neighbouring cells. There, magnetic flux bundles, ranging widely in size and strength (Harvey and Zwaan 1993), are produced by concentration of the small-scale magnetic field lines and form magnetic flux tubes through a sequence of processes described by the convective collapse theory (Spruit 1976, 1977; Schüssler 1990; Solanki 1996): Inside the magnetic field lines at the intergranular lanes, the plasma cools down by radiation and undergoes a convective downflow. This results in a drop in the inside plasma pressure. The higher plasma pressure in the surrounding squeezes the magnetic bundle and increases the concentration of the magnetic field lines in the bundle. The excess of the magnetic strength suppresses the convection within the bundle and eventually the magnetic pressure inside the tightly clumped magnetic bundle balances the outside plasma pressure (see Fig. 1.6 in Jafarzadeh 2013). These bundles of magnetic field lines cover almost 5\% of the solar surface and form magnetic flux tubes with magnetic strengths as high as 1-2 kG (Solanki et al. 2006).

At the intersections of the solar surface and the strong magnetic flux tubes, with magnitudes approximately between $10^{20} \mathrm{Mx}$ and $3 \times 10^{22} \mathrm{Mx}$ (Schrijver and Siscoe 2010), the very high magnetic pressure at the center of the thick flux tubes (with radii larger than 
local pressure scale height), prohibits the plasma motions across the flux tubes. Since the main source of energy at and slightly below the solar surface is the transported energy from the convection zone, the solar surface appears to be darker, compared to the surrounding, at the regions where large enough magnetic flux tubes emerge. These dark pores are referred to as sunspots. The strong concentration of the magnetic field lines at the locations of the sunspots (and sunspot groups), generate bipolar regions with enhanced emission in a wide spectral range, known as active regions (ARs). The solar areas surrounding the ARs were historically called the quiet Sun. A more uptodate definition is that, the solar regions outside ARs covering "all closed magnetic field regions" (Aschwanden 2005), are referred to as the quiet Sun.

One of the main characteristics of the solar photosphere is the large-scale flow of plasma material with a horizontal structure similar to the granulation. These large-scale motions, which systematically displace a group of granulation cells in a convective manner, are known as supergranulation (Leighton et al. 1962; Simon and Leighton 1964), with spatial scale of $\sim 30 \mathrm{Mm}$ and life-time of $\sim 20 \mathrm{~h}$ (Leighton et al. 1962). The magnetic flux is concentrated at the boundaries between supergranulation cells and forms the so-called magnetic network pattern. This pattern divides the quiet Sun into two components, the network and the internetwork regions (see Sec. 1.3.2).

\subsubsection{Quiet Sun magnetic field}

The quiet Sun was traditionally believed to posses no or very low magnetic activity. However, new studies in the last decade have given a different view to the quiet Sun magnetic properties, suggesting that the quiet Sun magnetic field dominates the magnetic flux of the full Sun even during the periods of solar maximum activity (Sánchez Almeida 2003; Sánchez Almeida and Martínez González 2011; Trujillo Bueno et al. 2004). Contrary to the active regions, whose magnetic properties are relatively well understood, the origin and properties of the quiet Sun magnetic field is still not so clear. The detection of the quiet Sun magnetic field is difficult since it is not as organised as that of the active regions, with their highly concentrated magnetic flux tubes.

However, measurements of the solar magnetic vector field (Lites et al. 1994) are providing a wider view of the complex solar magnetism. In this respect, recent observational studies such as Domínguez Cerdeña et al. (2003), Jin et al. (2009) and Lites et al. (2008), as well as simulations and theoretical efforts like those of Cattaneo (1999), Vögler and Schüssler (2007) and Rempel (2014) have revealed a very magnetically rich quiet Sun photosphere with the magnetic field strength in the intergranular cells, ranging between a few Gauss up to $130 \mathrm{G}$. Today, there is a consolidated agreement on the existence of small-scale magnetic fields in the quiet photosphere, however, their origin is still not well understood. There are three main suggested scenarios for the origin of the quiet Sun magnetism. A view is that they are originated from the decaying active regions (Spruit et al. 1987). Sánchez Almeida and Martínez González (2011) argue that this does not seem to be a proper explanation because of the large differences in the time-scales of the active region and quiet Sun magnetic evolution. The other two groups consider the interaction between the turbulence and the magnetic field, a turbulent dynamo, as the driver of the small-scale magnetic fields. However, what makes them distinct from each other is the depth in which the turbulent dynamo operates. While some scientists think that the turbulent dynamo is 
operating in the entire convetion zone (Stein and Nordlund 2002; Thornton and Parnell 2011; Stenflo 2012), some others believe that it exists only in the solar surface (Title and Schrijver 1998; Cattaneo 1999; Schüssler and Vögler 2008; Danilovic et al. 2010b).

\subsubsection{Local dynamo}

In the new picture of the quiet Sun magnetism, the photosphere is covered with a mixture of small magnetic elements of opposite polarities, a pattern sometimes referred to as Salt and pepper (when observed in high resolution magnetograms; Schrijver and Zwaan (2000)). These magnetic elements bound together with small-scale magnetic fluxes that are lying in the lower chromosphere and form the so-called magnetic carpet (Title and Schrijver 1998). It is thought that the magnetic field being tangled by turbulence, as a result of photospheric granulation, is responsible for the generation and maintenance of this small-scale magnetic structuring (Henoux and Somov 1991, 1997; Sánchez Almeida and Martínez González 2011). This mechanism in known as "local dynamo" (Cattaneo 1999; Miesch 2005; Brandenburg and Subramanian 2005; Vögler and Schüssler 2007; Rempel 2014) and, contrary to the global dynamo, it is only related to the quiet Sun and is time-invariant (Stenflo 2012). Note that, the existence of the local dynamo has not been observationally proved and is more referred to in the context of numerical simulations (Stenflo 2012, but see Danilovic et al. 2010a and Buehler et al. 2013). Martínez Pillet (2013) has written a thorough review on small-scale magnetic properties of the quiet Sun and all possible dynamo mechanism that can generate them.

\subsubsection{Magnetic field in the solar atmosphere and the network pattern}

The kG magnetic flux tubes emerging through the magnetic network are in nearly vertical orientation because of their buoyancy (Schüssler 1986). These vertical flux tubes continually alter the upper solar atmosphere and produce non-homogeneous brightenings. In the quiet Sun, these brightenings produce a "honeycomb-shaped pattern" (Schrijver and Siscoe 2010), co-spatial with the underlying magnetic network (to a high degree), which is observable in almost all chromosphere and transition region UV and VUV emission lines. The bright boundaries and the dark interiors of this pattern are referred to as the network and internetwork regions, respectively. The intensity enhancement in the network regions is presumably a result of magnetic heating, however the mechanism is not yet known precisely. In the transition region, the contribution of low-lying loops in the formation of the network is also discussed by Dowdy et al. (1986). Fig. 1.3, taken from de Wijn et al. (2009), illustrates a patch of network structure in the photosphere and the chromosphere. The images show how the supergranulation lanes in the photosphere underlie the chromospheric network.

As one climbs up in the solar atmosphere from the photosphere to the corona, the gas pressure and the magnetic pressure decay exponentially, with scale height of several $100 \mathrm{~km}$, up to chromospheric altitudes. From this point up, the drop in the magnetic pressure lags behind the drop in the gas pressure. The plasma $\beta$, which was initially greater than 1 in the photosphere (outside of magnetic flux tubes), starts to drop below 1, resulting in horizontal expansion of the flux tubes and formation of the so-called magnetic canopy, a term that was introduced by Gabriel (1976) to explain the UV emission lines originated 

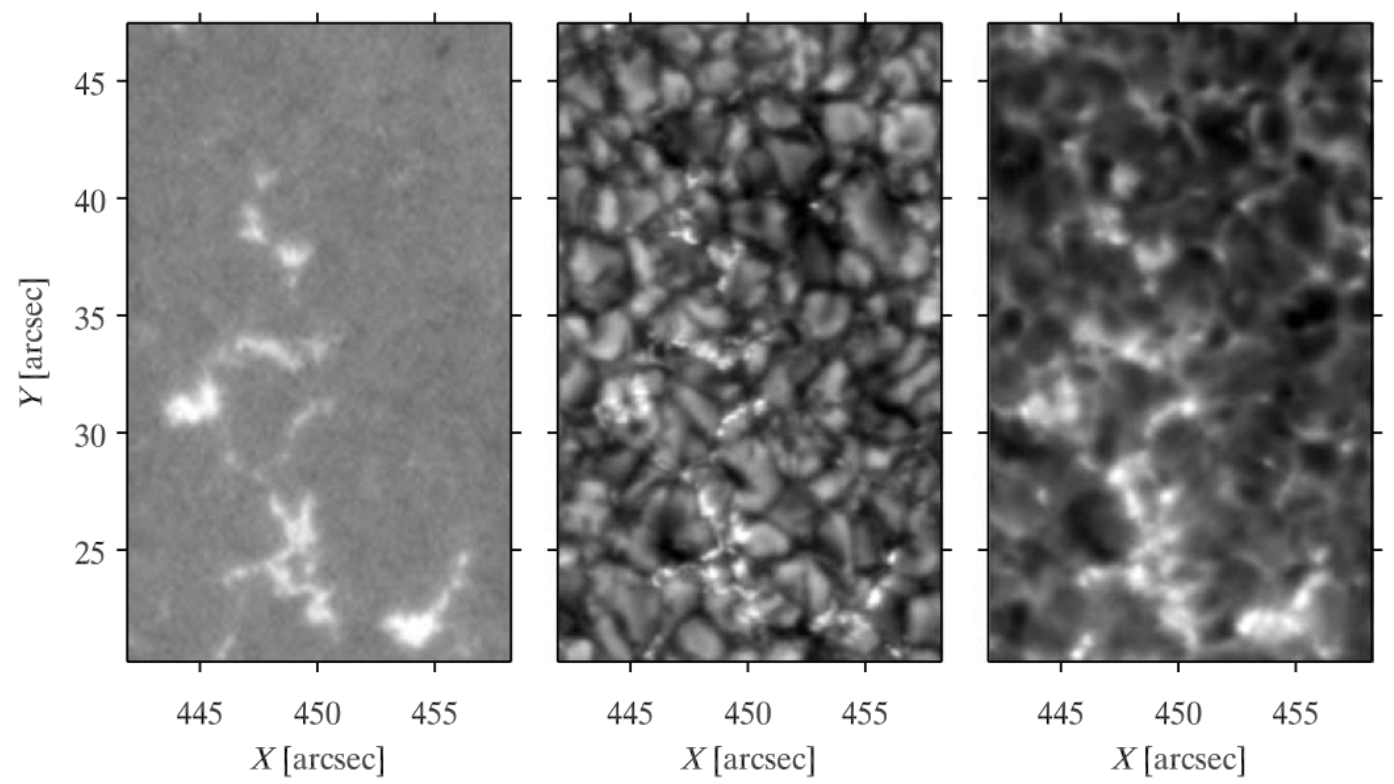

Figure 1.3: A network structure as seen in the photospheric Fe I $630.2 \mathrm{~nm}$ line-of-sight magnetogram (left) and G-band (midle), and in the chromospheric $\mathrm{Ca}$ II $\mathrm{H}$ line (right). The images are taken with Hinode/SOT on 2007 March 30 (figure taken from de Wijn et al. 2009).

in the upper solar atmosphere. In the quiet chromosphere, the filling factor is relatively small and the canopy base is located at about $1000 \mathrm{~km}$ above the surface. Over the active regions, the expansions occurs at much lower heights, in the photosphere. Higher in the transition regions, the lateral expansion of the magnetic tubes is very large, extending over an underlying supergranulation cell. The solar magnetic field is reviewed in details by Solanki et al. (2006) and Wiegelmann et al. (2014).

The horizontal expansion of the magnetic flux tubes manifests itself in the increase of network coverage and thickness in the upper layers compared to the photosphere. The photospheric magnetic flux tubes that shape the magnetic network have diameters of about $0.1^{\prime \prime}-0.2^{\prime \prime}$ (when observed in the G-band ${ }^{1}$ ), while due to the expansion of flux tubes, the counterpart chromospheric network appears to be more diffuse with widths of about 1.5"-10" (Goodman 2000). Patsourakos et al. (1999) and Gontikakis et al. (2003), also showed that the size of the network elements in several chromospheric and transition region spectral lines, observed by CDS and SUMER (both aboard SOHO satellite), varies between $5^{\prime \prime}$ to $12^{\prime \prime}$. It was also shown by both studies that the network thickness is roughly constant in the chromosphere and transition region (considering the larger optical depth in the chromosphere which results in slightly thicker network), but increases drastically in the higher corona.

${ }^{1}$ A Fraunhofer "line" at $430.5 \mathrm{~nm}$ produced by hundreds of transitions of the $\mathrm{CH}$ molecule. 


\subsection{Heating mechanisms}

The physical mechanisms that lead to heating up the upper solar atmosphere, specifically the corona, into extremely high temperatures is one of the biggest questions in astrophysics. Here, without going into details, we will only give an overview to the proposed heating mechanisms thought to be responsible for these high temperatures. For detailed discussion the reader is referred to available reviews on the coronal heating problem, such as Narain and Ulmschneider (1996), Walsh and Ireland (2003), Klimchuk (2006) and Parnell and De Moortel (2012).

In a very wide frame, what we know about coronal heating is that the energy consumed to heat the corona initially originates from the convection zone, where a part of the thermal energy converts into mechanical and magnetic energy, a portion of which is then transported to the outer atmosphere and heats the corona. What are not well-understood are the generation of the energy, the transport of it and its dissipation in the atmosphere (Falconer et al. 2003). Depending on whether or not magnetic energy is involved, all suggested heating mechanisms can be divided into two main categories: hydrodynamic (HD) mechanisms, in the quiet chromosphere, and magnetohydrodynamic (MHD) mechanisms, in regions were plasma is embedded in magnetic field (Narain and Ulmschneider 1996).

In the photosphere, the plasma motions constantly move the footpoints of the coronal magnetic fields, exerting stress on magnetic field lines (resulting from slow change of magnetic configuration) or generating waves along the flux tubes (due to relatively fast MHD perturbations) (Klimchuk 2006). Therefore, as long as MHD mechanisms are considered and based on the time-scales involved (from generation of an energy carrier to dissipation of the energy in the corona), the heating mechanisms can be alternatively classified into two groups: alternating current (AC) heating mechanism (Roberts 2000), due to dissipation of MHD waves, and direct current (DC) heating mechanism (Priest and Forbes 2000), where the magnetic stress is dissipated through the reconnection mechanism. In the scope of this dissertation, we have a closer look at two of the suggested mechanisms. The nanoflare model, as an example of DC heating, which is believed to be the reason why the VUV radiative intensity from the quiet Sun has lognormal distribution (see Sec. 1.5), and the non-magnetic heating mechanism by acoustic waves, as a possible mechanism for heating the internetwork quiet chromosphere (see Sec. 1.4.2).

\subsubsection{Nanoflare model}

The term nanoflare was first introduced by Parker (1988) to explain the heating of the corona through a series of reconnection events, an idea that had been suggested previously by Levine (1974). Nanoflares are impulsive energy releases within a single magnetic strand (as compartments of a thicker kilogauss magnetic flux tube (Klimchuk 2006)), with an average energy of $10^{24} \mathrm{erg}$ (Klimchuk (2009), Reale (2010)). The convective-driven random motions of the photospheric plasma, constantly shuffle the footpoints of the coronal field lines, wrapping the neighbouring magnetic field lines around each other. This gradually increases the magnetic stress in the corona and as soon as the misalignment between the tangled field lines exceeds a critical angle (between $10^{\circ}$ and $20^{\circ}$ in active regions and quiet Sun, respectively; Klimchuk 2006), the braided field lines undergo a reconnection event. The magnetic reconnection (Fig. 1.4) is a result of the Ohmic dissipation (Parker 1972) 


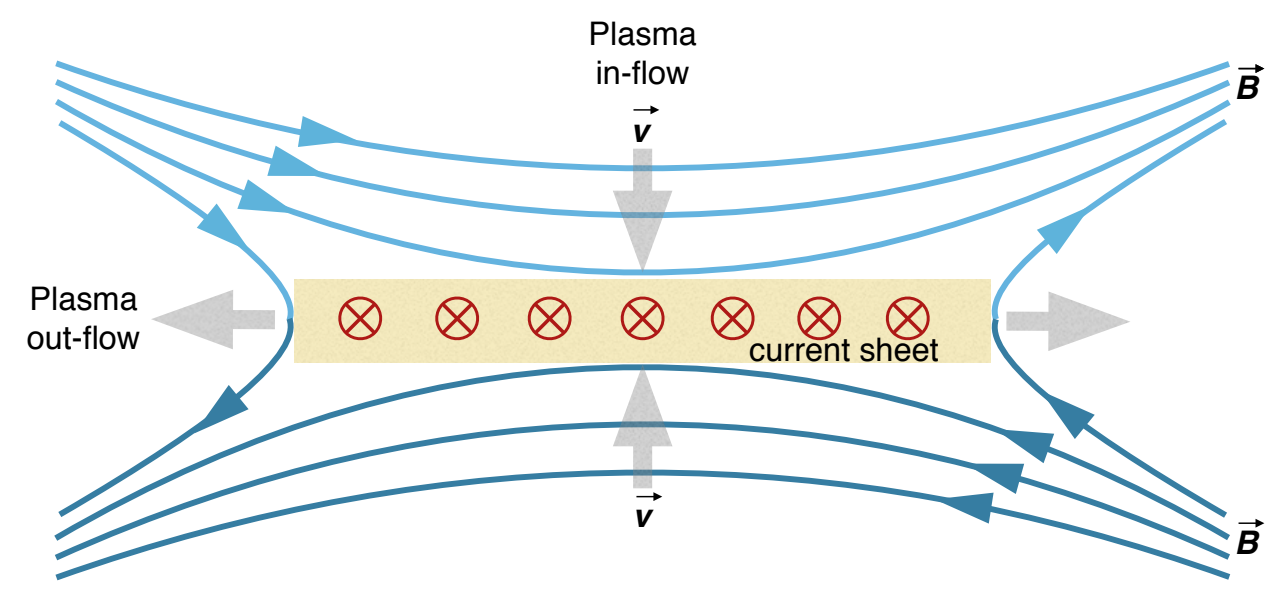

Figure 1.4: Simple model of magnetic reconnection.

of the very large electric currents at the place of tangential discontinuities, also known as current sheets (see Bowness et al. 2013 for a detailed review). In other words, whenever the gradient of the magnetic field across the current sheet exceeds a critical value, the magnetic field lines break and reconnect in a different connectivity configuration with a lower energy state. The extra magnetic energy is then released as heat.

Hudson (1991) showed that the frequency of nanoflare occurrence follows a power-law distribution in the form of $f(E)=f_{0} e^{-\gamma}$, where $E$ is the event energy and $f_{0}$ and $\gamma$ are constants. If $\gamma$ is less than 2 , it means that the high energy flares dominate the heating, and if it exceeds 2 , the heating is dominated by small scale events, or namely nanoflares. Different studies report a range of values for the exponent $\gamma$ (see table 1 in Narain and Pandey 2006); some finding small power-law index and hence challenging Parker's nanoflare model (Aschwanden and Charbonneau 2002 and references cited therein) and some supporting it by finding values larger than 2 (Georgoulis and Vlahos 1996, Benz and Krucker 1998). As shown by Pauluhn and Solanki (2007), the power law exponent is generally underestimated because the smaller events blend and form a near continuum background that leads to an underestimate of their numbers. This implies that $\gamma>2$ is more likely.

\subsubsection{Heating of the transition region}

Aschwanden (2008), counting a number of problems with nanoflares occurring in the corona, suggested that nanoflaring should happen in the transition region, where the misalignment between localized magnetic strands are stronger and the rate of flaring is higher. This idea had been previously proposed by Büchner et al. (2004), who showed that the reconnection events, and consequently resistive heating due to current sheets, happen already in the chromosphere and TR. An evidence for this scenario is that almost all observed EUV nanoflares are located in the TR (Aschwanden and Parnell 2002). In a more recent study by Testa et al. (2013), investigation of the EUV images from the High-resolution Coronal Imager (Hi-C), led the authors to find rapid small-scale variability at the top of the TR over active regions. They deduced that the highly variable brightenings were indeed due to nanoflare events. 
In a model introduced by Pauluhn and Solanki (2007), the authors produced a synthetic time series of EUV radiance with the assumption that EUV radiation from the quiet Sun is entirely generated due to occurrence of macro and nanoflares. By statistically comparing the modelled EUV radiance (being produced by nanoflares with power-law exponent larger than 2) with the observed radiance from TR and coronal emission lines (from SUMER), they showed that both synthetic and observed radiances have indeed very similar distributions; both following the lognormal distribution function. The radiance distribution of the quiet Sun is discussed in Sec. 1.5.

Patsourakos et al. (1999) classifies the TR emission formation into two main groups. According to Feldman $(1983,1987)$, the unresolved fine structures that do not reach coronal temperatures and that contribute very little to chromospheric emission produce the bulk of TR radiation. A similar picture was given by Rabin and Moore (1984), who argued that the numerous cool-loops lying in the TR are responsible for the emission from the lower TR, but in the upper TR, the radiation originates from the coronal magnetic funnels extending from the network regions on the photosphere. This latter view was originally suggested by Gabriel (1976) and is the main concept of the second school of thoughts about TR heating. In this regard, Athay (1982) suggested that the downflow of the hot gas from corona into TR through magnetic funnels triggers a large part of TR radiation. Roberts (2000) also pointed out that almost half of the coronal heating energy is transported back into the TR by thermal conduction. The formation of many TR emission lines is therefore believed to be linked to coronal back-heating.

\subsubsection{Acoustic waves and chromospheric heating}

The photospheric convective motions that exert stress on the magnetic field lines, simultaneously generate different forms of waves, propagating upward in the solar atmosphere and transporting energy to the outer layers (Klimchuk 2006). Among all the possible waves, Biermann (1946) and Schwarzschild (1948) proposed that the dissipation of the acoustic waves is one of the main sources of heating in the chromosphere and corona (Fossum and Carlsson 2006). These waves are generated by pressure fluctuations, namely turbulence, due to granular motions at the top of the convection zone, through the "lighthill mechanism" (Lighthill 1952). The generation and propagation of the acoustic waves has been studied in detail by Stein $(1967,1968)$ and more recently by Carlsson and Stein (1997), Wedemeyer-Böhm et al. (2007) and Schröder et al. (2012). A comprehensive review on observational studies of acoustic waves is also provided by Rutten and Uitenbroek (1991).

The energy input to the solar atmosphere at the top of the convection zone is several times $10^{4} \mathrm{Wm}^{-2}$ (Narain and Ulmschneider 1996). However, only a fraction of this energy budget passes through the chromosphere and may eventually contribute to heat the corona. The residual energy is responsible for the temperature gradient in the chromosphere. The radiative energy loss in the chromosphere, depending on whether it originates from the quiet Sun or active region, is in the range of $4 \times 10^{3}-10^{4} \mathrm{Wm}^{-2}$ (Narain and Ulmschneider 1996). Similar values was also reported by Ulmschneider (1974), Athay (1976) and Vernazza et al. (1981).

By modelling chromospheric lines and continuum radiation, Ulmschneider and Kalkofen (2003) evaluated various heating possibilities and deduced that the Joule heating and thermal conductivity are not sufficient to compensate the chromospheric cooling and their 
contribution is small enough to be ignored, and that at least in the quiet Sun internetwork regions, the dominant heating mechanism is the acoustic heating. By studying the observations from Transition Region And Coronal Explorer (TRACE), Fossum and Carlsson (2006) estimated the acoustic energy flux to be $255 \mathrm{Wm}^{-2}$, at least one order of magnitude less than what was expected. It was later shown by Wedemeyer-Böhm et al. (2007) that the spatial resolution of TRACE is responsible for the hidden acoustic flux. Moreover, Kalkofen (2007) pointed out that a large portion of the network emission is also due to acoustic waves, an evidence for which was provided by Rezaei et al. (2007). The largest amount of acoustic flux observed so far, was reported by Bello González et al. (2010), using the high-resolution spectropolarimetric data from IMaX/SUNRISE.

In a wider picture, the constant increase of the temperature in the chromospheres of the Sun-like stars, is usually attributed to the mechanical heating with acoustic waves (Athay and Thomas 1961, Kuperus et al. 1981, Narain and Ulmschneider 1996). Schrijver (1987) argued that the heating of the chromospheres of the late-type stars ${ }^{1}$ has two main contributions: a temperature dependant basal flux, and a magnetic flux, which depends on the rotation of the star. He suggested that the basal flux is a lower limit flux originated from the lower chromosphere and is due to purely acoustic heating. Later works by Schrijver et al. (1989), Buchholz et al. (1998) and Fawzy et al. (2002) also approved the heating of the quiet chromosphere by acoustic waves. However Schrijver (1995) pointed out that the small scale magnetic fields can also play a role. These small scale magnetic fileds, as discussed in Sec. 1.3.1.1, can be due to the existence of a local dynamo. In fact, Bercik et al. (2005) pointed out that a hypothetical local dynamo in a non-rotating plasma is indeed capable of producing the basal flux observed by Schrijver (1987) and, therefore, can be regarded as an alternative to the acoustic heating mechanism. Regardless of which of these mechanisms generate the basal flux, the important point is that they are both independent of the solar global dynamo and, hence, are invariant with the magnetic activity cycle of the Sun.

Overall, thanks to state-of-the-art simulations and modern technological improvements in observational instruments, the understanding of the quiet Sun magnetism has been significantly changed during the recent years. Fig. 1.5, adopted from Wedemeyer-Böhm et al. (2009), illustrates the extremely complex picture of the quiet solar atmosphere, characterized by many different phenomena, such as low-lying loops in the upper photosphere, current sheets, shock waves, etc. This schematic sketch shows how the magnetic field lines emerge through the network regions, divide the solar atmosphere into the canopy and sub-canopy domains and expand in the TR and corona. "Fluctosphere", as defined by Wedemeyer-Böhm and Wöger (2008), is the region of low magnetic fields between the photosphere and the chromosphere, which is dominated by shock waves. Note that this sketch is a 2D picture, implying that it is still a very simplified picture of the quiet Sun atmosphere.

\footnotetext{
${ }^{1}$ Stars of class $\mathrm{K}$ and $\mathrm{M}$ with a surface temperature lower than that of the Sun. These two types of stars make up roughly $88 \%$ of the main-sequence stars in the solar neighborhood (Ledrew 2001).
} 


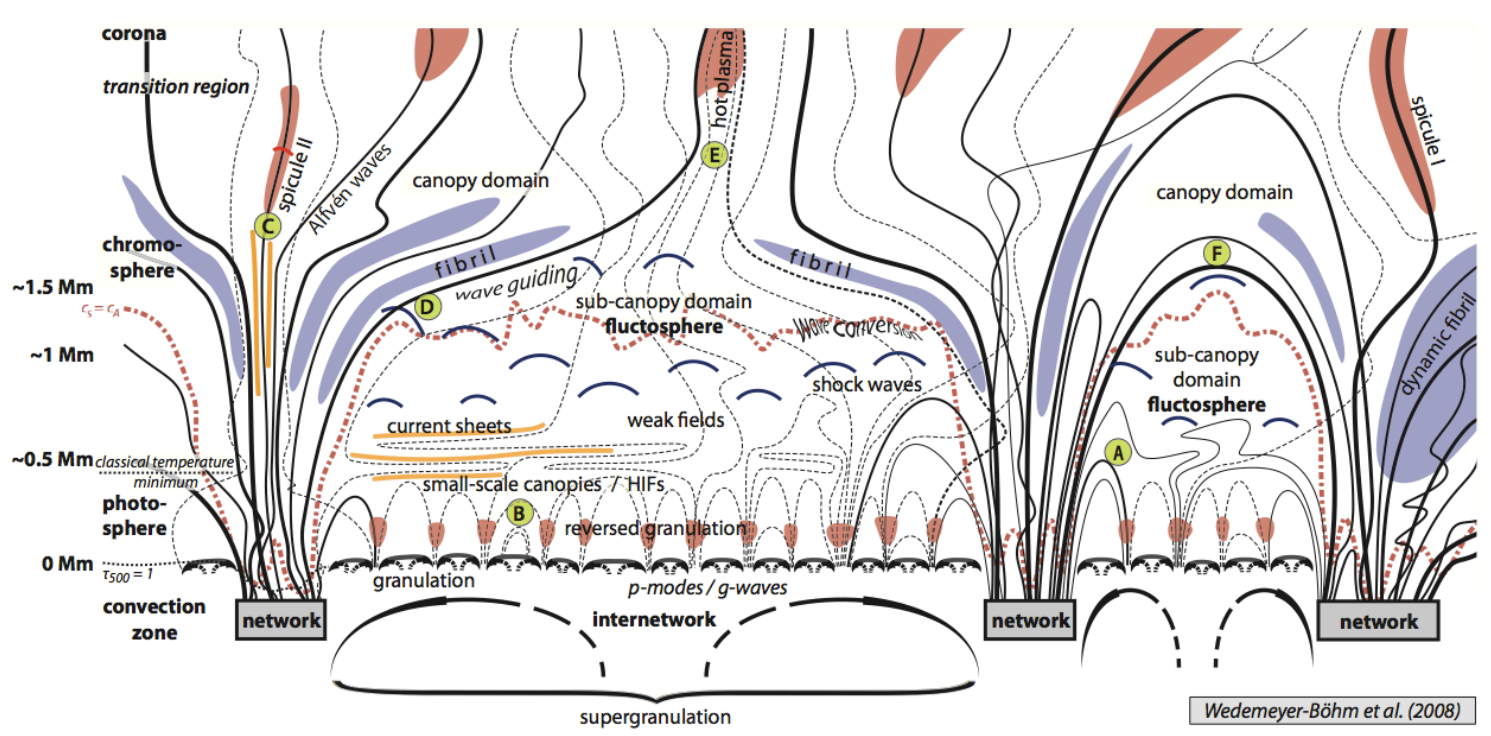

Figure 1.5: Schematic sketch of various phenomena in the quiet Sun atmosphere (figure is taken from Wedemeyer-Böhm et al. 2009).

\subsection{Radiance distribution of the Quiet Sun}

The solar radiation delivers crucial information about the physical processes in the Sun, and the heating mechanisms are no exceptions. In this regard, it is of interest to study the radiance distribution of the quiet Sun, since it can provide information on the prevalent heating mechanisms.

By definition (see Sec. 1.1.3 in Yung 2003 and Sec. 4.2 in Fu 2006), the radiant energy per time per unit solid angle per unit source area and at a given wavelength range, $\lambda$ to $\lambda+d \lambda$, is called spectral intensity or monochromatic radiance $I_{\lambda}$, and is formulated as:

$$
I_{\lambda}=\frac{d E_{\lambda}}{d t d \lambda d \Omega d A \cos \theta} .
$$

where $d E_{\lambda}$ is the radiant energy, $d A \cos \theta$ is the effective area and $d \Omega$ is the differential solid angle. The integral of the monochromatic radiance over the range of wavelengths is then called the intensity or radiance I, with SI unit W.sr $r^{-1} \cdot m^{-2}$.

Various studies have shown that the radiance distribution of the quiet Sun in the chromosphere and transition region has a skewed shape, with a pronounced peak at relatively low intensities and an extended tail at higher intensities. This skewed distribution has been modelled mainly by either a sum of two Gaussian functions (Reeves 1976, Gallagher et al. 1998) or a single Lognormal function (Griffiths et al. 1999, Pauluhn et al. 2000, Fontenla et al. 2007), each representing a different hypothesis about the source of the radiation in quiet Sun regions. According to Pauluhn et al. (2000), these two models imply different heating scenarios. A two-component function assumes two intrinsically different heating mechanisms for radiation originating in the solar network and internetwork, while a single-component model assumes similar (at least in terms of their spatio-temporal scales) heating mechanisms for both network and internetwork. They showed that despite its fewer 
free parameters, a lognormal function reproduced the observed distributions significantly better than the sum of two Gaussians.

Based on the work by Bogdan et al. (1988), who showed that the size of sunspot umbrae is lognormally distributed, Griffiths et al. (1999) suggested that the lognormal distribution of the quiet Sun TR radiance is associated with "small, fragmenting magnetic elements" (see Bogdan 1992 and Berger et al. 1995). The connection between the umbrae size distribution and TR radiance is based on the consideration that, the lognormal distribution of the umbrae is assumed to be a result of the fragmentation of the large magnetic flux tubes at photospheric levels (Pauluhn et al. 2000), which, on smaller scales, are in turn believed to be responsible for the EUV radiance in the chromosphere and TR, at least over the network regions (Nindos and Zirin 1998; Sivaraman et al. 2000; Loukitcheva et al. 2009). Note that Schrijver et al. (1989) found that the photospheric magnetic field and chromospheric EUV radiance are not always following each other and their relationship is not one-to-one. This is more of an issue in the internetwork regions, where according to some studies such as Remling et al. (1996) and Lites et al. (1999), there is no or very little connection between magnetic field and internetwork brightening. To this it should be added that it can be argued theoretically that the weak internetwork magnetic field is hardly capable of producing EUV brightening (Pauluhn et al. 2000). In case of the chromosphere, although the heating by nanoflares is plausible by some studies (Büchner et al. 2004). Fontenla et al. (2007) also argued that the lognormal form of the radiance distribution is attributed to the dissipation of the small-scale magnetic free-energy (which, as they interpreted, is due to photospheric dynamos) through plasma instabilities such as the Farley-Buneman instability (Fontenla 2005).

All together, relating the lognormal distribution of the quiet Sun radiance to the magnetic flux fragmentation and/or local photospheric dynamos is not as straightforward. Other heating mechanisms such as acoustic and MHD waves are also considered as potential candidates. Nonetheless, although the physical mechanism leading to the skewed form of the radiance distribution in the quiet Sun is still a question, the common agreement today is that it follows a lognormal distribution function.

\subsubsection{Lognormal distribution}

It is believed that a system variable can be modelled with a lognormal distribution if it is a multiplicative product of many independent forces acting on that system (Grönholm and Annila 2007, Limpert et al. 2001). These expressions can be applied to solar physics through, e.g., the nanoflare heating model which, as already explained in Sec. 1.4.1, is a potential mechanism for coronal heating. The consistency between the lognormal distribution of TR radiation in the quiet Sun and the nanoflare model has been studied by Pauluhn and Solanki (2007), Bazarghan et al. (2008) and Tajfirouze and Safari (2012).

The lognormal distribution function can be mathematically defined by replacing the independent variable (here intensity $I$, as defined by eq. 1.2) in the normal distribution function with its logarithm. In other words, if $\log (I)$ is normally distributed, the variable $I$ is lognormally distributed:

$$
P(I)=\frac{N_{0}}{\sigma I \sqrt{2 \pi}} \exp \left(-\frac{(\ln (I)-\mu)^{2}}{2 \sigma^{2}}\right) .
$$


The above equation shows a lognormal probability density $P(I)$ as a function of intensity. Here $\mu=\langle\ln (I)\rangle, \sigma=\sqrt{\operatorname{Var}(\ln (I))}$ and $N_{0}$ is the normalization factor. The $\mu$ and $\sigma$ are the mean (or median) and standard deviation of $\ln (I)$. Note that we use natural logarithm in the definition of the lognormal function. Using another base to the logarithm only rescales the fitting parameters, but eventually returns the same family of distributions (Limpert et al. 2001). The back-transformed parameters are then defined as

$$
\mu^{*}=e^{\mu}, \sigma^{*}=e^{\sigma}
$$

and called scale parameter $\left(\mu^{*}\right)$ and multiplicative standard deviation or shape parameter $\left(\sigma^{*}\right)$, respectively (Limpert et al. 2001). Using these two main parameters, a set of statistical parameters can be defined to express different features of a given distribution. Some examples are as follows:

$$
\begin{gathered}
\gamma=\left(e^{\sigma^{2}}+2\right)\left(\sqrt{e^{\sigma^{2}}+1}\right), \\
C V=e^{\sigma^{2}}-1, \\
\bar{I}=e^{\mu+\sigma^{2} / 2}, \\
M O D=e^{\mu-\sigma^{2}},
\end{gathered}
$$

where $\gamma, C V, \bar{I}$ and $M O D$ are skewness, coefficient of variation, mean and mode of the distribution. Among these, skewness and coefficient of variation depend only on $\sigma$, and are the measures of asymmetry and normalized dispersion of the distribution, respectively, while the mean and mode values are dependant on both $\sigma$ and $\mu$. In the case of the normal distribution, the mean, mode and median parameters are basically the same. But when the distribution deviates from being a Gaussian and becomes asymmetric, these parameters diverge from each other and reflect different properties of the distribution.

Fig. 1.6, depicts the lognormal distributions of a given random variable $I$ with different $\sigma^{*}$ values of 1.5, 2 and 4, and a fixed $\mu^{*}$ value of 5, in linear and logarithmic scales. The dashed and dotted lines indicate the mean and mode of each plot and the red lines in both panels mark the scale parameter $\mu^{*}$, which is constant for all plots. The distributions being plotted in the logarithmic scale have Gaussian forms. This is equivalent to plotting the distribution of the logarithm of the random variable in the linear scale, which by definition (Eq. 1.3), has a normal distribution.

It is obvious from the figure that the distribution with the largest $\sigma^{*}$ (plotted in green), is the most asymmetric one. This can also be realized from the "logarithmic scale" panel, where the mode, mean and the scale parameters are furthest from each other. The opposite, is the distribution with the smallest $\sigma^{*}$ (plotted in black), in which the mode, mean and the scale parameters are approaching together and, therefore, the distribution can be said to be more similar to a normal distribution. Hence, the larger the shape parameter $\sigma^{*}$ is, the more asymmetric (and skewed) the distribution will be. 

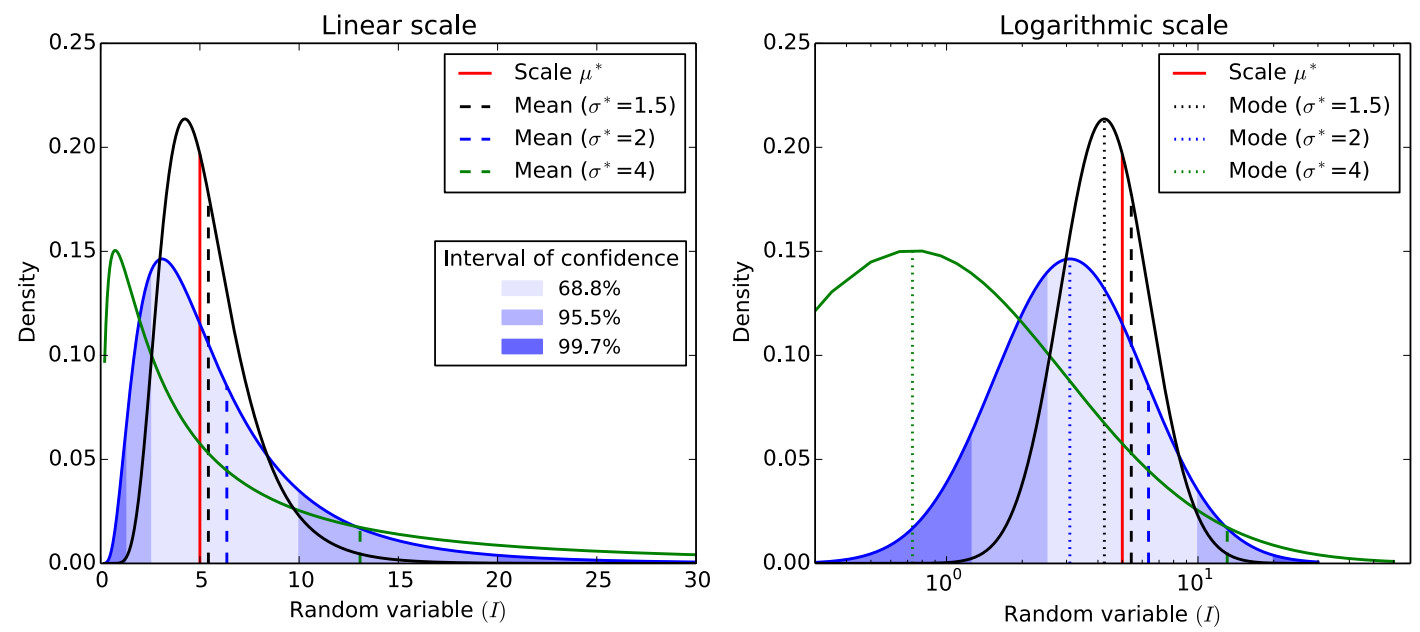

Figure 1.6: Lognormal distributions for three data samples with different $\sigma^{*}$ values and the same $\mu^{*}$ value. In the right panel, the distributions are plotted on a logarithmic scale, and therefore have Gaussian form. The dashed and dotted lines correspond to means and modes of the distribution, respectively, and the red line indicates the scale parameter $\mu^{*}$. Different shades of blue mark the intervals of confidence for the distribution with $\sigma^{*}=2$, where the lightest shade consists of $68.3 \%$ of the distribution population. This, together with the sightly darker shade, include $95.5 \%$ of the data points. All shades of blue contain $99.7 \%$ of the data. These are the data which are lying in the interval of $\left(\mu^{* \times}, \sigma^{* 3}\right)$.

In addition, we know that for normally distributed data, $68.3 \%, 95.5 \%$ and $99.7 \%$ of the data population lie in the intervals of $\mu \pm \sigma, \mu \pm 2 \sigma$ and $\mu \pm 3 \sigma$, respectively. The corresponding boundaries for a lognormally distributed data are $\mu^{* \times}, \sigma^{*}, \mu^{* \times}, \sigma^{* 2}$ and $\mu^{*} \times \sigma^{* 3}$, where, in analogy to the \pm symbol (plus or minus by), the ${ }_{\text {, symbol denotes times }}$ or divide by (Limpert et al. 2001). These intervals are shown in Fig 1.6, for the distribution with $\sigma^{*}$ equal to 2 (plotted in blue).

From the definitions of $\mu$ and $\sigma$, one can see that the shape parameter $\sigma^{*}$ of a given distribution is invariant with respect summation and multiplication of the independent variable $I$.

\subsection{1-year sunspot cycle}

In Sec. 1.3, we briefly explained how the dynamo in the convection zone produces the solar magnetic field (of which, sunspots are the most evident manifestation) through the so-called $\alpha-\omega$ effects. The appearance of the sunspots on the surface of the Sun follows a periodic pattern, through which the number of sunspots rises and falls to successive maxima and minima, respectively, with an average 11-year cycle. The fact that the sunspots are the locations of the strong magnetic fluxes; means that the sunspot cycle is actually a magnetic cycle. The distribution of the sunspots changes by latitude throughout a cycle. They start to emerge at higher latitudes (about $20^{\circ}$ to $40^{\circ}$ ) by the beginning of the cycle and migrate toward the equator as the cycle proceeds. The equator-ward drift of the sunspots was referred to as "Spörer's Law of Zones" by Maunder (1903) and generates the famous 
"butterfly diagram". At the same time that the sunspots drift toward the equator, the weaker magnetic fluxes outside of the sunspots (the remnants of the active region fluxes Upton and Hathaway 2014) move toward the poles. Prior to the transport, the magnetic fluxes from opposite polarities are being separated in the active latitudes due to Joy's law (Hale et al. 1919). Hence, the higher and lower latitudes are dominated by the fluxes of opposite polarities, so that the higher latitude fluxes always possess a polarity that also differs from that of the magnetic field previously occupying the polas. As a result, the poleward transported magnetic fluxes gradually cancel out the original magnetic fields in the poles. Close to the maximum of the sunspot cycle, the polar magnetic fields start to reverse polarity and by the end of the cycle the new polar fields reach their highest intensity (Nandy 2012). These newly intense polar magnetic fields, in turn, build up a new poloidal magnetic dipole during the minimum of the sunspot cycle, which again will be subjected to differential rotation and meridional flow and triggers the next sunspot cycle. Fig. 1.7, taken from Hathaway and Upton (2014), illustrates the migration of the magnetic flux and flipping of the polar magnetic polarity.

The topic of solar activity cycle is very vast and includes many different fields which are out of the scope of this dissertation. The reader is therefore referred to the numerous publications on solar activity cycle, such as Charbonneau (2005), Petrovay (2010), Solanki and Krivova (2011) and Usoskin (2013). However, what still needs to be briefly mentioned here, is that the variations in the sunspot number is only one aspect of the solar 11-year cycle. The periodic emergence of magnetic flux tubes that alter the outer solar atmosphere, also modifies the output electromagnetic spectrum of the Sun in a periodic way. However, different wavelength regimes of the solar spectrum respond differently to the solar cycle. For instance, the total solar irradiance (TSI) varies by $0.1 \%$ over a full cycle (note that, 2-3 times larger changes in shorter time-scales have also been observed Fröhlich (2004)). The variations in the shorter wavelengths is much larger, reaching up to $100 \%$ in XUV and VUV parts of the solar spectrum (Fröhlich 2004, see also Fig.1 in Ermolli et al. 2012).

As pointed out briefly in the summary, the solar cycle and its induced variations in the solar output energy affect the Earth in different ways. The most controversial issue, in this respect, is the effect of the solar output on the constantly increasing global temperature of the Earth. Lockwood et al. (2009) and Lockwood et al. (2010) showed that neither the increased global temperature nor the cold recent winters in Europe, are due to the solar cycle. These studies were mainly based on the observations of various solar parameters, mainly TSI value, during the last solar cycle (cycle 23) and its following anomalous activity minimum in 2008.

\subsubsection{Anomalous solar cycle 23}

The minimum in solar activity between cycles 23 and 24 was different in many aspects compared to other recent solar minima. It lasted longer, with nearly twice as many spotless days than the previous eight minima, and was particularly deep and inactive (Schrijver et al. 2011, Tapping and Valdés 2011). Moreover, the Sun's interplanetary field in the recent minimum was approximately at half of the value it had during solar minimum 21 and was at its lowest level since the beginning of the measurements in 1963 (Lockwood et al. 2009). This result is consistent with a halving of the Sun's open flux (Vieira and Solanki 2010). A particularly interesting question is how the Sun's radiative output reacted 


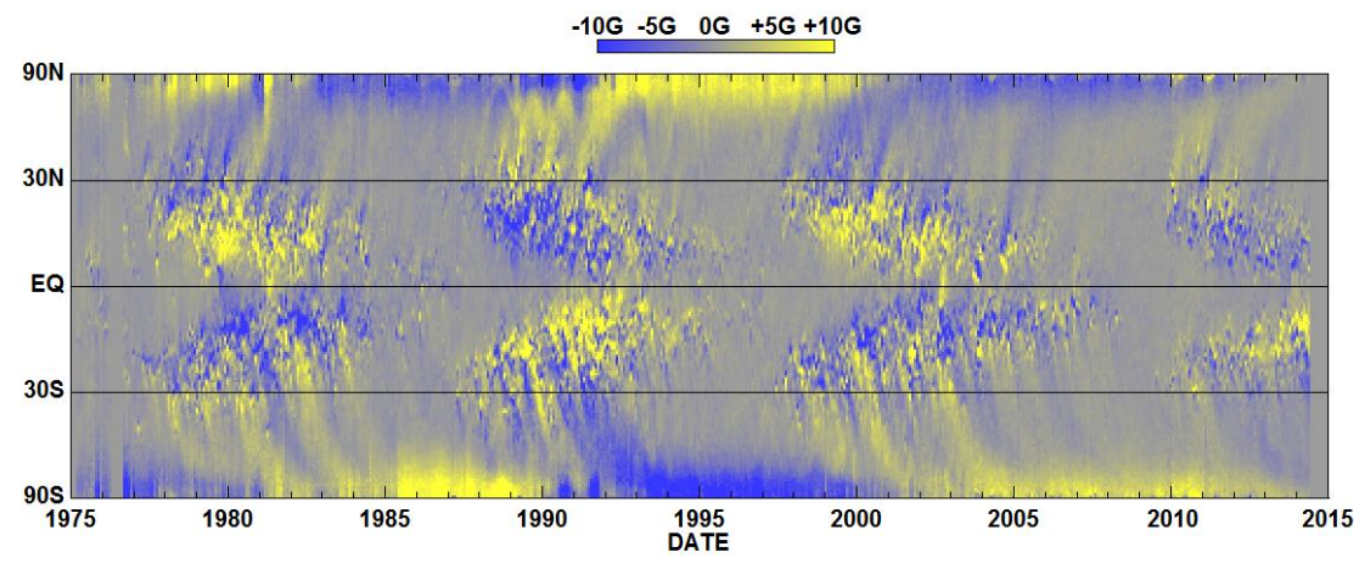

Figure 1.7: A magnetic Butterfly diagram of cycles 21 to 24 . The diagram illustrates the emerging of the magnetic fluxes and their migration toward the poles (image taken from Hathaway and Upton (2014)).

to the particularly low level of activity.

Of the three main composites of total solar irradiance (TSI); ACRIM (Willson and Mordvinov 2003; Scafetta and Willson 2009), PMOD (Fröhlich 2006; Fröhlich 2009) and RMIB (Mekaoui and Dewitte 2008), two (PMOD and the ACRIM) display a decrease in the TSI value during the minimum between cycles 23 and 24 compared to the previous minimum (Woods 2010; Fröhlich 2012). This decrease is independently obtained by models of solar irradiance which assume the evolution of the surface magnetic field drives solar irradiance variability (Solanki et al. 2013). Thus for the TSI, which is formed mainly in the photosphere (Solanki and Unruh 1998), the evidence suggests a significant difference between the two minima. The differences between these three composite TSI records have been explained in detail by Woods (2010) and Fröhlich (2012).

The situation is less clear-cut for radiation formed in the upper atmosphere. The solar radio microwave flux at $10.7 \mathrm{~cm}$ is commonly regarded as an activity indicator of the upper chromosphere and lower corona. The $10.7 \mathrm{~cm}$ radio flux has two main sources: thermal bremsstrahlung emission and gyroradiation. Therefore, the enhancements in the temperature and the magnetic field of the Sun, as results from emerging sunspots, increase the $10.7 \mathrm{~cm}$ flux, producing a high correlation between the sunspot number and solar radio flux. However, in line with anomalous trends in some other solar activity indices during the last solar cycle, this well-pronounced correlation seems to have changed during solar cycle 23 (Tapping and Valdés 2011), especially close to the solar minimum in 2008 (Svalgaard and Hudson 2010). In addition, two different studies by Fröhlich (2011) and Tripathy et al. (2010) show a very slight decrease (within 4\%) in the amplitude of the $10.7 \mathrm{~cm}$ flux over the last solar minimum compared to the minimum in 1996. This is tiny compared with the changes displayed by other activity indicators between the two activity minima.

Other solar activity indices, such as EUV flux (Solomon et al. 2010; Woods 2010) and the Mg II index (Weber et al. 2013), also exhibit a similar decreasing trend over solar minimum 23. The measurements from the SOHO Solar EUV Monitor (SEM) (Judge et al. 1998), for instance, reveal a $15 \%$ decrease with $6 \%$ uncertainty in the irradiance of the spectral band from 26 to $34 \mathrm{~nm}$ over minimum 23 with respect to the minimum 22 (Didkovsky et al. 2010). 
Beside all these anomalous changes which are basically concerning the solar spectrum, Upton and Hathaway (2014) pointed out that the solar meridional flow was significantly faster close to the solar minimum 23/24 in comparison to the meridional flow preceding the previous minimum in 1995, by almost 20\%. Furthermore, Schrijver and Liu (2008) found that the faster diverging meridional flow close to the end of the cycle results in a weaker magnetic dipole, or poloidal field, at the beginning of the following cycle. It was shown by de Toma (2012) and Wang et al. (2009) that the polar magnetic fields above $60^{\circ}$ latitude were approximately $40 \%$ weaker than the three previous solar minima. 


\section{Instrumentation}

As the main purpose of this dissertation is to study the "long-term" variations in the Sun's VUV irradiance, the observational data were chosen such that they satisfy two main criteria. The first one was the period of time covered by the data, long enough to cover at least the last two solar minima. The second, was that the data should be fairly homogeneous and include various emission lines in the VUV spectral range, originating from a specific atmospheric layer of the Sun. Such data would allow comparing the emissions from different atmospheric layers over a long period of time. The only instruments that could satisfy these criteria were the ones on board the Solar and Heliospheric Observatory (SOHO).

$\mathrm{SOHO}$ is a joint international project between ESA and NASA. The SOHO spacecraft was launched on 1995 December 2 and started its nominal science phase in April 1996. Although SOHO was originally planned as a two-year project, it is still operating after 18 years. The purpose of the SOHO mission was to study the Sun from its core to the outer atmosphere and the solar wind, by the means of its twelve instruments. Concerning the science of the solar atmosphere, a main goal of the mission was to help scientists to understand how the corona is heated and why it exists. SOHO has made it possible to pursue this goal by providing unique information about the physical properties of the solar plasma in the outer atmosphere.

Among all SOHO instruments, the observations from the Coronal Diagnostic Spectrometer (CDS; Harrison et al. 1995) and the Extreme-ultraviolet Imager (EIT; Delaboudinière et al. 1995) are those that better fit the above two selection criteria and, thus, were dominantly used in the current study. In the following, a brief overview of these two instruments is presented.

\subsection{Coronal Diagnostic Spectrometer (CDS)}

The CDS instrument was designed and constructed through an international collaboration between various institutions and universities in Europe and in the US, led by the Rutherfor Appleton Labratory (RAL) in the UK. The members of the CDS international team and their contributions are listed in Harrison et al. (1995). This article also contains detailed technical and operational information of the instrument. Here, a very brief overview of some of the main instrument characteristics is presented. For a thorough discussion on the instrument features and data analysis, the reader is referred to the main CDS article by Harrison et al. (1995), as well as a series of software notes provided by the CDS team ${ }^{1}$.

${ }^{1}$ http://solar.bnsc.rl.ac.uk/software/notes.shtml 
a.

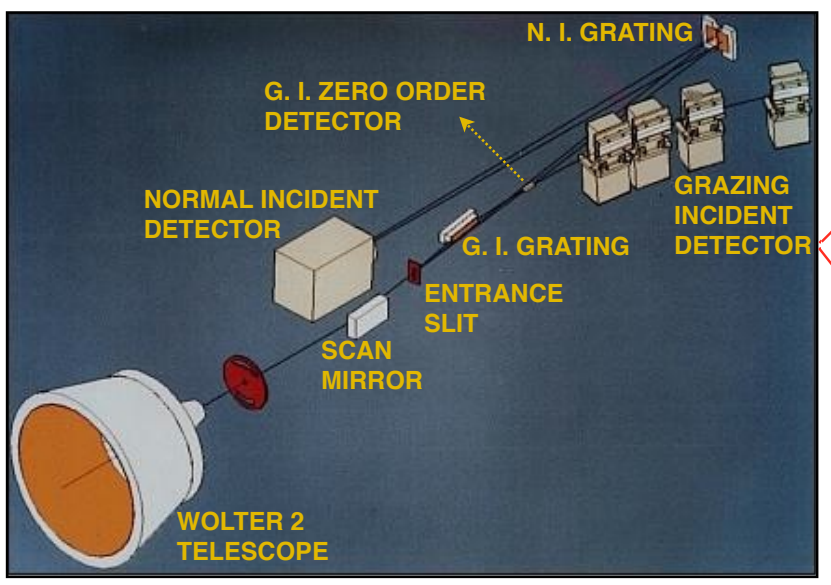

b.
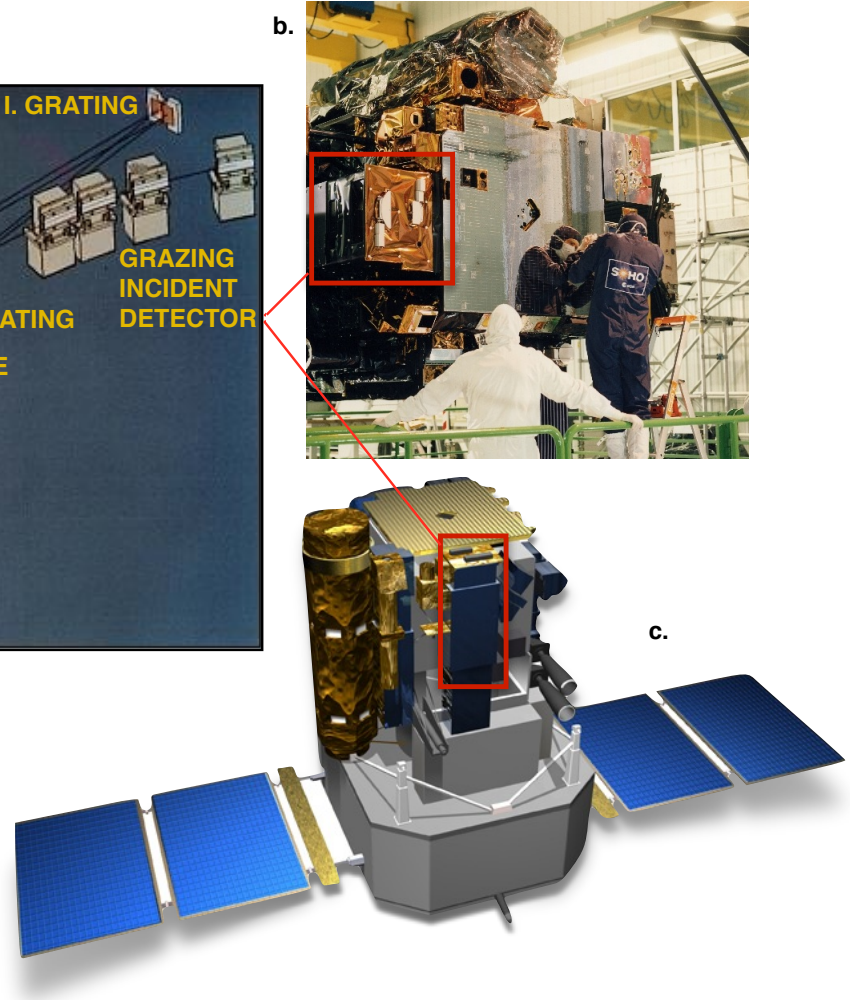

Figure 2.1: a) Optical layout of the CDS instrument (image credit: RAL). b,c) SOHO satellite. The location of the CDS instrument aboard SOHO is marked by the red rectangles (image credit: SOHO -ESA \& NASA).

The CDS instrument is a double spectrometer, consisting of a normal incidence spectrometer (NIS) and a grazing incidence spectrometer (GIS). As illustrated in Fig. 2.1, the optical layout of the instrument consists of a Wolter-Schwarzschild type II telescope with a field of view of 240", a scan mirror, an assembly of six slits (three for each spectrometer) and the already mentioned spectrometers. Note that only one spectrometer can be used at a time. Two apertures, each with a closeable door in the panel in front of the telescope, define the two independent optical paths. A combination of six legs supports the instrument and rotates it through an angle of $\pm 0.75^{\circ}$. This mechanical system, which is referred to as the Offset Pointing System (OPS), permits the instrument to be pointed to different regions of the Sun and lower corona. This mechanism, together with the field of view of the telescope allow CDS to observe any location within \pm 48 arcminute from Sun centre. The operation and calibration of OPS is studied by Thompson and Carter (1998).

The solar image from the telescope is reflected on the entrance slits by a scan mirror at a grazing incidence of $\sim 5^{\circ}$. The scan mirror can rotate within $\pm 0.3^{\circ}$ perpendicular to the slit direction (oriented along the solar central meridian in nominal attitude conditions), with step sizes equivalent to $2^{\prime \prime}$ on the Sun. The spectral maps are therefore constructed in a piece-by-piece fashion out of a defined number of mirror steps (exposures). The slits are located at the focal plane of the telescope and are oriented perpendicular to the dispersion plane. The field of view provided by the slits is listed in Table 2.1. All NIS slits are 240" in length. Also, the pinhole aperture that is used by GIS can rotate within $\pm 120^{\prime \prime}$ along the north-south direction of the Sun. Therefore, the combination of the scan mirror rotation, 
Table 2.1: CDS main optical characteristics.

\begin{tabular}{l|ll}
\hline General & Weight & $100 \mathrm{~kg}$ \\
& Overall Length & $1.7 \mathrm{~m}$ \\
& Pointing & $48^{\prime}$ from disc centre \\
& Raster Step size & E-W: $2.032^{\prime \prime}$ \\
& & N-S: $1.016^{\prime \prime}$ \\
\hline Telescope & Eff. Focal length & $257.831 \mathrm{~cm}$ \\
& Field of view & $240^{\prime \prime}$ \\
& FWHM & $<2^{\prime \prime}$ \\
& Aperture & NIS: $34.3 \mathrm{~cm}^{2}$ per grating \\
& & GIS: $47 \mathrm{~cm}^{2}$ \\
& Plate scale & $12.5 \mu \mathrm{m} /{ }^{\prime \prime}$ \\
\hline Slits & NIS & $2^{\prime \prime} \times 240^{\prime \prime}, 4^{\prime \prime} \times 240^{\prime \prime}, 90^{\prime \prime} \times 240^{\prime \prime}$ \\
& GIS & $2^{\prime \prime} \times 2^{\prime \prime}, 4^{\prime \prime} \times 4^{\prime \prime}, 8^{\prime \prime} \times 50^{\prime \prime}$ \\
\hline NIS CCD & Opr. Temperature & $-70^{\circ} \mathrm{C}$ \\
& Dimension & $1024 \times 1024$ pixels ${ }^{2}$ of $21 \mu \mathrm{m}$ \\
& Quantum efficiency & $20 \%$ \\
& Dark current & $<0.01$ photon $/$ pixel $/ \mathrm{s}$ \\
\hline Wavelength & NIS-1 & $30.8-38.1 \mathrm{~nm}$ \\
range & NIS-2 & $51.3-63.3 \mathrm{~nm}$ \\
& GIS-1 & $15.1-22.1 \mathrm{~nm}$ \\
& GIS-2 & $25.6-33.8 \mathrm{~nm}$ \\
& GIS-3 & $39.3-49.3 \mathrm{~nm}$ \\
& GIS-4 & $65.6-78.5 \mathrm{~nm}$ \\
\hline \multirow{2}{*}{ Oon } & \multicolumn{1}{c}{ Spectral lines studied } \\
\hline & 58.43 & $250,000 \quad(\mathrm{chromosphere})$ \\
& &
\end{tabular}

slits length (for NIS) and pinhole aperture movement (for GIS), results in CDS rasters with dimensions (without re-pointing) up to $240^{\prime \prime} \times 240^{\prime \prime}$, for both spectrometers. The observational data used in this study are entirely from the normal incidence spectrometer, therefore in the following, a more detailed discussion on the NIS detector is given.

The NIS spectrometer has a Rowland circle design; the beam coming from the slits hit a pair of toroidal gratings at a mean angle of $7.9^{\circ}$ and is reflected toward the NIS detector almost along the diameter of the Rowland circle. The gratings (with the groove densities of 2400 and 4000 lines/mm for shorter and longer wavelengths, respectively) disperse the incident radiation onto the NIS detector in two bands, covering the wavelength range from $30.8 \mathrm{~nm}$ to $38.1 \mathrm{~nm}$ (NIS-1) and from $51.3 \mathrm{~nm}$ to $63.3 \mathrm{~nm}$ (NIS-2). The emission lines observed by CDS are listed in tables I to IV of Harrison et al. (1995). The NIS detector, known as Viewfinder Detector Subsystem (VDS) is an intensified CCD camera 

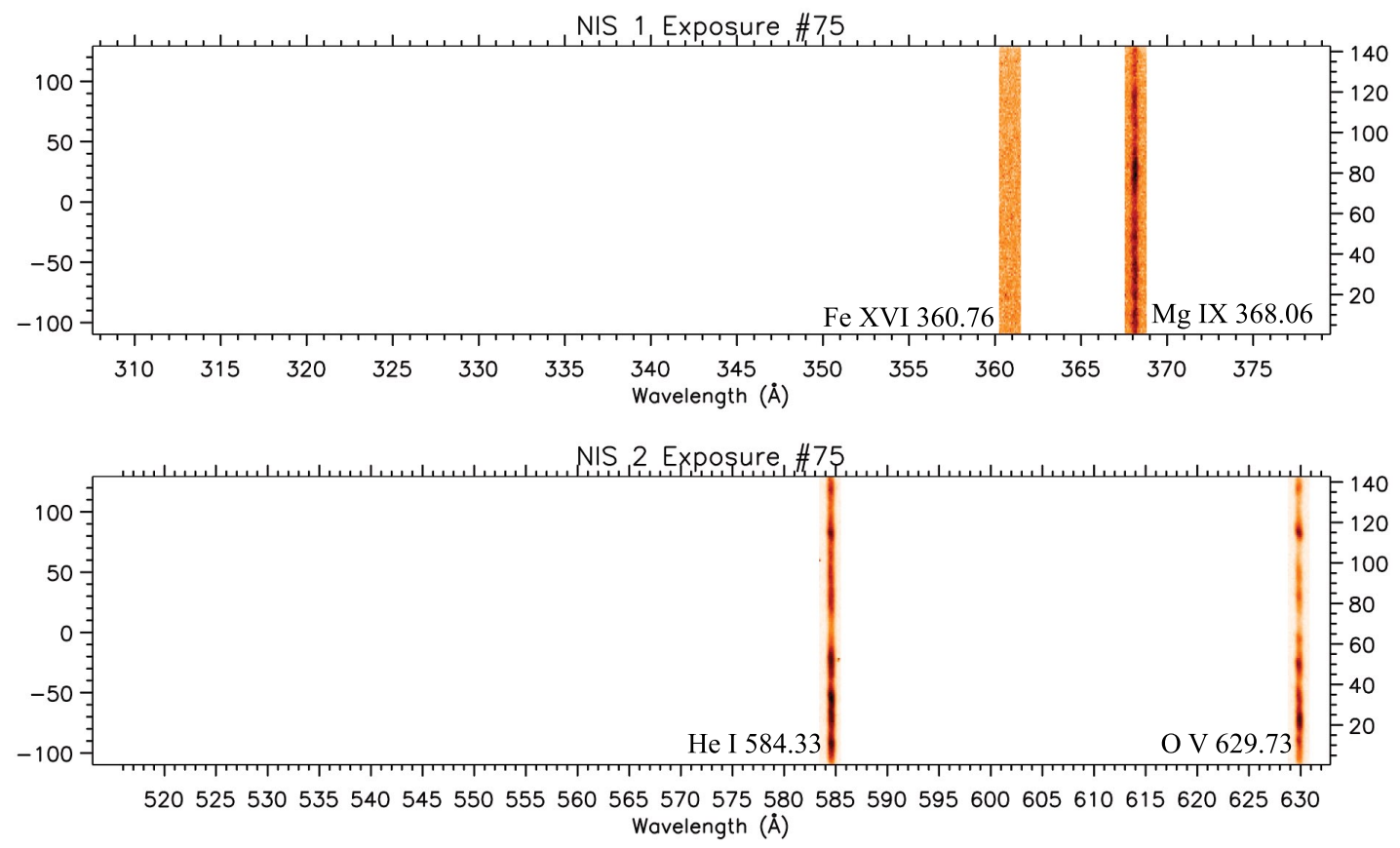

Figure 2.2: NIS full-detector spectrum from a single exposure. As explained in the context, each observation consists of 120 exposures. The spectrum shown here is the exposure no.75 of a given raster taken on $1996 \mathrm{Sep} 1$. The position of four spectral lines is shown across the wavelength bands. Each band consists of 1024 pixels across the detector in $x$ direction and 143 pixels along the detector (corresponding to detector Y indices 336 to 478 for NIS-2 and 584 to 726 for NIS-1).

(Thompson et al. 1992), consisting of three compartments: an image intensifier, a lens and a CCD. A combination of a MicroChannel Plate (MCP) and a phosphor converter, intensifies and converts the EUV radiation to visible light, which is later focused on the CCD via a custom lens system. The CCD contains $1024 \times 1024$ pixels $^{2}$ with dimensions of $21 \mu \mathrm{m}$. About a quarter of the pixel rows (286 rows) in the middle of the CCD is used to represent the field of view along the solar Cartesian $y$ direction. These pixel rows are split into the two spectral bands, NIS-1 and NIS-2, such that in each exposure, the spatial information along the solar $y$ direction is projected on to 143 pixel rows along each NIS wavelength bands. Fig. 2.2 exhibits a full detector spectrum from a single exposure. As examples, four spectral windows corresponding to four emission lines are marked in the figure, two on each band. As already explained above, each band has 143 pixels in the detector $y$ direction, which corresponds to $240^{\prime \prime}$ on the Sun, or in other words, the spatial pixel size in $y$ direction is $1.68^{\prime \prime}$. The spectral pixel size (across the detector $x$ direction) ranges from $0.07 \AA$ at $310 \AA$ to $0.118 \AA$ at $630 \AA$. The spectral resolution was initially $0.35 \AA$ for NIS- 1 and $0.5 \AA$ for NIS- 2 in terms of full-width at half-maximum (FWHM) (Del Zanna et al. 2010; Del Zanna 1999).

In June 1998 the contact with SOHO was lost due to operational errors. Prior to the launch of SOHO, the operation of the CDS instrument had been tested in the temperature 


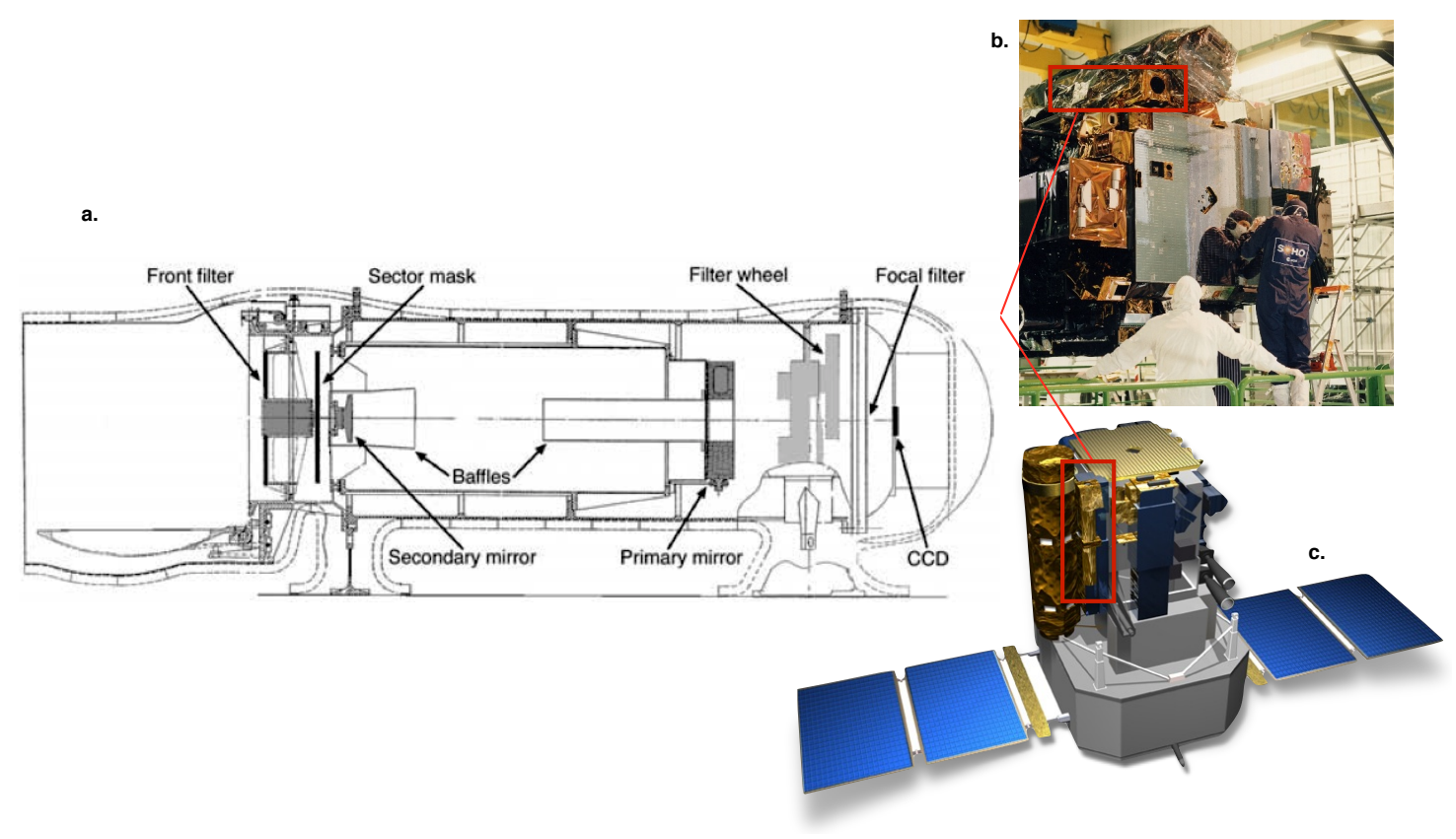

Figure 2.3: a) Schematic sketch of EIT subsystem (taken from Auchère et al. 2001). b,c) SOHO satellite. The location of the EIT instrument aboard SOHO is marked by the red rectangles (image credit: SOHO -ESA \& NASA).

range of $0^{\circ}$ to $40^{\circ} \mathrm{K}$, but during the four months between the SOHO loss and complete recovery, the instrument experienced extreme temperature conditions and was maintained at $\sim 100 \mathrm{~K}$. After recovery in September 1998, the NIS spectral profiles were broadened and asymmetric, resulting in a reduction in the spectral resolution (see Pauluhn et al. 2001).

\subsection{Extreme-ultraviolet Imaging Telescope (EIT)}

In-line with the SOHO objectives in terms of understanding the solar corona, the primary scientific goal of EIT is to study the evolution of the temperature and dynamic structure of the inner corona and transition region within $1.5 R_{\odot}$. The EIT instrument was designed and manufactured through an international collaboration between NASA/Goddard Space Flight Center, USA, the Royal Observatory of Belgium and the Institut d'Astrophysique Spatiale in Orsay, France. In the following a brief introduction to the instrument's main characteristics is presented. For more in-depth discussions on the technical and operational features of the instrument, the reader is referred to the main instrument article by Delaboudinière et al. (1995) as well as a complementary paper on the EIT preflight photometric calibration by Dere et al. (2000).

EIT is a normal incidence, multilayer, wide-field Ritchey-Chretien telescope with a field of view of $45 \mathrm{arcmin}^{2}$. It provides full-disc images in four different narrow passbands (listed in Table 2.2) and covering temperatures in the range of $6 \times 10^{4} \mathrm{~K}$ to $3 \times 10^{6} \mathrm{~K}$. The EIT schematic subsystem and its position on board SOHO is shown in Fig. 2.3. A more simplified picture of the instrument's optical layout is also illustrated in Fig. 2.4. The optical layout consists of the following parts: three filter sets (the entrance filters, filters 


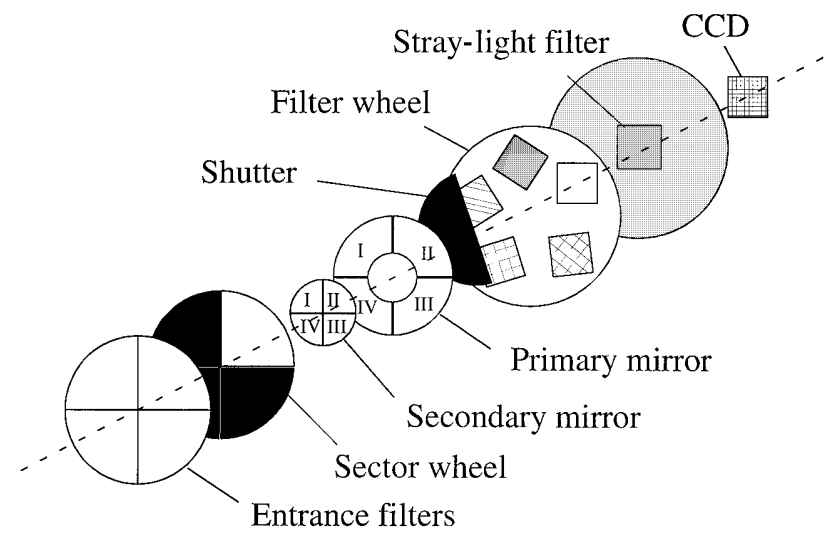

Figure 2.4: Optical elements of EIT (image from Dere et al. 2000).

Table 2.2: EIT bandpasses and temperature coverage (Delaboudinière et al. 1995).

\begin{tabular}{llll}
\hline$\lambda(\mathrm{nm})$ & Ion & Peak Temp. $(\mathrm{K})$ & Observational Objective \\
\hline 17.1 & Fe IX-X & $1.3 \times 10^{6}$ & Corona and TR boundary; Coronal holes \\
19.5 & Fe XII & $1.6 \times 10^{6}$ & Quiet Corona outside Coronal holes \\
28.4 & Fe XV & $2.0 \times 10^{6}$ & Active Regions \\
30.4 & He II & $8.0 \times 10^{4}$ & Chromospheric network; Coronal holes \\
\hline
\end{tabular}

implanted on a filter wheel and stray-light filter right in front of the CCD), the primary and secondary mirrors with multilayer coating on each matched quadrant, a sector wheel to select one quadrant of the telescope at a time, and the CCD.

The multilayer coatings on the mirrors are made of alternating layers of molybdenum (Mo) and silicon (Si). The coating on each quadrant are tuned to different wavelengths, such as to obtain very high reflectivity at the desired wavelength passband (see Delaboudiniere et al. 1989). The entrance filter, whose functionality is to reject heat, is composed of a celluloid film $(70 \mathrm{~nm}$ ) with aluminium coatings on both sides (each $150 \mathrm{~nm}$ ), supported on a metal grid. The filter wheel contains four filters and an open slot. Of the four filters, one is pure aluminium and one is similar to the entrance filter. These are used to block the long wavelength emissions. The other two are opaque screens coated partially with aluminium, such to prevent the radiation from reaching the upper or lower one third of the CCD.

The detector used in EIT includes a $1024 \times 1024$ pixel $^{2}$ back-illuminated CCD, with pixel size of $21 \mu \mathrm{m}$ and spatial sampling of 2.6". The quantum efficiency of the CCD in the wavelength range given in Table 2.2 varies between 0.036 at $17.14 \mathrm{~nm}$ and 0.27 at $30.38 \mathrm{~nm}$. The CCD is designed to operate in $-80^{\circ} \mathrm{C}$ in order to decrease the dark current. This temperature is provided by a passive radiator isolated from the solar radiation and facing deep space. Prior to flight, the optical alignment of the instrument was optimized for $20^{\circ} \mathrm{C}$. However, since the internal temperature of the optical bench, when in space, is not stable over time, the thermal design of the internal structure was calculated and tested for temperatures below $20^{\circ} \mathrm{C}$.

To reject stray-light, the CCD is isolated from the telescope section by being confined inside a vacuum chamber. The intersection between the detector and telescope sections is 


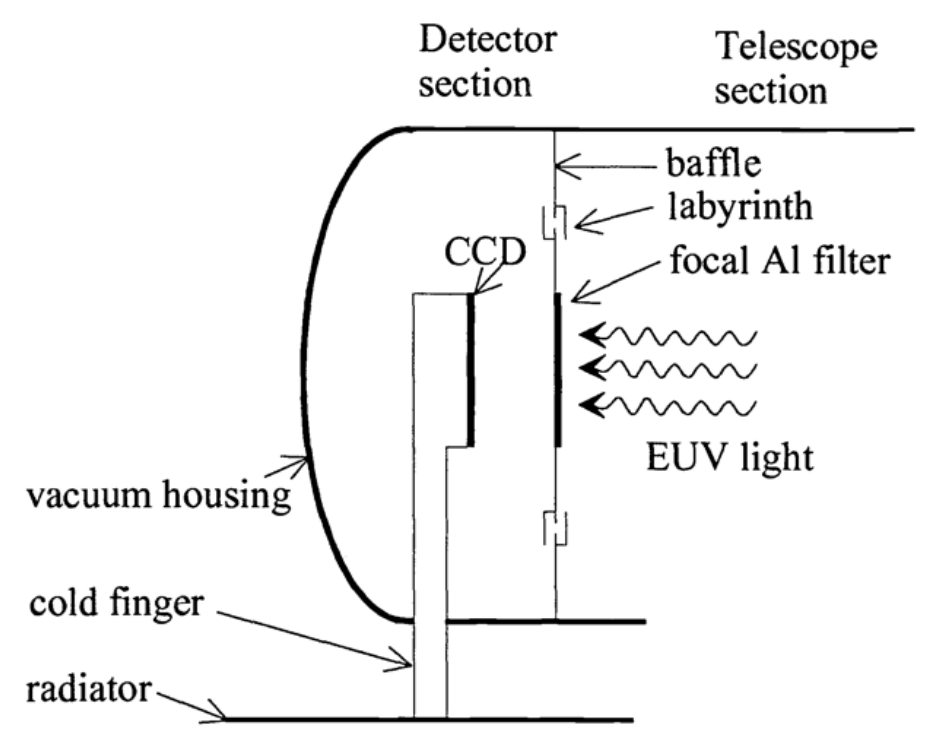

Figure 2.5: Configuration of the detector section (image from Defise et al. 1998)

a stray-light baffle which is equipped with a stray-light filter from pure aluminium with the thickness of $150 \mathrm{~nm}$. Fig. 2.5 shows the detector section set-up. The closed design of the detector chamber results in a much longer time in evacuating the gas and contaminants trapped inside the CCD chamber (Defise et al. 1998). The contamination and effect of stray-light has always been a source of error for EIT observations. The effect of stray-light in EIT images has been discussed by Auchère et al. (2001).

EIT is the only instrument aboard SOHO that does not have a flight computer, but uses the computer and the electronics in the $\mathrm{LASCO}^{1}$ electronic box (LEB). In addition, no pointing mechanism is implemented for EIT and the instrument is directly bolted on the spacecraft. Hence, EIT is the SOHO pointing reference and other on board instruments align themselves with EIT images. Table 2.3, taken from Delaboudinière et al. (1995), gives an overview of the EIT main characteristics.

${ }^{1}$ The Large Angle Spectroscopic COronagraph aboard SOHO (Brueckner et al. 1995). 
Table 2.3: EIT main characteristics (from Delaboudinière et al. 1995).

\begin{tabular}{|c|c|}
\hline \multicolumn{2}{|c|}{ Telescope (Ritchy-hretien) } \\
\hline Mirrors & Multilayer-coated Zerodur \\
\hline Multilayers & Mo-Si tuned to different $\lambda \mathrm{s}$ in each quadrant \\
\hline Bandpass centers & $17.1(\mathrm{~nm}), 19.5(\mathrm{~nm}), 28.4(\mathrm{~nm})$ and $30.4(\mathrm{~nm})$ \\
\hline Bandpass selection & Rotatable open quadrant shutter \\
\hline Effective focal length & $165.2 \pm 0.2 \mathrm{~cm}$ \\
\hline Primary diameter & $12 \mathrm{~cm}$ \\
\hline Geometrical area per quadrant & $13 \mathrm{~cm}^{2}$ \\
\hline \multicolumn{2}{|c|}{$\mathrm{CCD}$ detector (cooled at about $-80^{\circ} \mathrm{C}$ ) } \\
\hline Architecture & $\begin{array}{l}\text { Three phase, multiphase-pinned, } \\
\text { back-illuminated, enhanced EUV sensitivity }\end{array}$ \\
\hline Array size & $1024 \times 1024$ pixel $^{2}\left(45^{\prime} \times 45^{\prime}\right)$ \\
\hline Pixel size & $21 \mu \mathrm{m}$ square $\left(2.6^{\prime \prime}\right)$ \\
\hline Full well & $>150,000$ electrons \\
\hline Readout rate & $5 \times 10^{4} \mathrm{pixel} / \mathrm{sec}$ \\
\hline On-chip summation & e.g. $2 \times 2$ pixel $^{2}$ and $4 \times 4$ pixel $^{2}$ \\
\hline Full frame readout time & $21 \mathrm{sec}$ \\
\hline \multicolumn{2}{|r|}{ Filters } \\
\hline Entrance Filter & $150 \mathrm{~nm} \mathrm{Al} / 70 \mathrm{~nm}$ cellulose $/ 150 \mathrm{~nm} \mathrm{Al}$ \\
\hline Filter wheel: Pos. 0 & $150 \mathrm{~nm} \mathrm{Al}$ \\
\hline Pos. 1 & $150 \mathrm{~nm} \mathrm{Al} / 70 \mathrm{~nm}$ cellulose $/ 150 \mathrm{~nm} \mathrm{Al}$ \\
\hline Pos. 2 & CCD bottom third blocked, top $150 \mathrm{~nm} \mathrm{Al}$ \\
\hline Pos. 3 & Open, no filter \\
\hline Pos. 4 & CCD top third blocked, top $150 \mathrm{~nm} \mathrm{Al}$ \\
\hline CCD stray-light filter & $150 \mathrm{~nm} \mathrm{Al}$ adjacent to $\mathrm{CCD}$ \\
\hline
\end{tabular}




\section{Observations and Data reduction}

In the introduction chapter, it was explained that the primary objective of this dissertation is to study the long-term variations in the EUV radiation of the quiet Sun during the last solar cycle, with the main focus on the solar minima. In Chapter 2, it was pointed out that the only instruments providing long enough observational data in the wavelength range of interest, are CDS and EIT, both aboard SOHO. In this thesis I mainly employ the data from $\mathrm{CDS} / \mathrm{NIS}$, therefore, in the following, the CDS/NIS data selection and reduction procedure is discussed in details. The EIT data are briefly explained in Chapter 6, outlook.

\subsection{Observational data}

A large number of studies (observation sequences) have been defined before and after launch of CDS. Harrison et al. (1995) categorized all these studies into three main areas: 1. synoptic sequences for solar monitoring, 2. calibration and monitoring sequences, and 3. scientific sequences. In this dissertation, we used the NIS synoptic study (the so-called study program SYNOP). It is made up of daily observations along the central meridian of the Sun from the north to the south pole of the Sun. Each run of SYNOP consists of a series of 9 raster scans, each covering an area of $240^{\prime \prime} \times 240^{\prime \prime}$ of the solar disc. Observations were taken employing the $2^{\prime \prime} \times 240^{\prime \prime}$ slit and by scanning along the solar $x$-direction in 120 separate exposures. Each scan contains $120 \times 143$ spectra, each sampling an area of $2^{\prime \prime}$ (in the solar x-direction) by 1.68" (in the solar y-direction). In order to concentrate on the quiet Sun area close to disc-center, we only analysed the central rasters of each set of 9 raster scans, with solar $y$ pointings between $-120^{\prime \prime}$ and $+120^{\prime \prime}$. However, in addition to the central rasters, two other rasters from the north and south hemispheres (raster scans number 2 and 6 from each set) were also analysed to check the environment of the central raster for detecting the appearance of the active regions near the disc-center ( $\operatorname{see} \mathrm{Sec} 4.6$ ).

The complete data set analysed during this thesis work, covers a period of time from 1996 April 1 to 2011 May 21 and consists of more than 4000 raster scans of daily measurements. As the main goal of this investigation is to look for any statistical variations in the radiance distribution of the quiet Sun during the last two solar minima, we chose a time span of 1 year during each minimum and did a comparative study over various statistical parameters (as will be described in Capter 4). The 1-year periods were chosen to be from 1996 April 1 to 1997 April 1 (minimum 22) and from 2008 July 1 to 2009 July 1 (minimum 23). 


\subsection{CDS/NIS data structure}

The CDS/NIS data are stored in a 3D structure with the dimensions of $x, y$, and $\lambda$, corresponding to solar $x$ and $y$ directions and the wavelength, meaning that each spatial pixel of a given raster scan contains a spectrum. Prior to any analyses, it is necessary to convert the 3-dimensional CDS raw data into meaningful physical parameters, or more specifically, intensity values. After reducing the data, the spectra at individual spatial pixels will be fitted with Gaussian functions and an intensity value, defined as the integrated area under the Gaussian fits, will be assigned to corresponding pixels. This way the wavelength dimension will be eliminated and a 2D intensity image will be produced. These steps are discussed in the following section.

\subsection{Data reduction}

The content of this section is mainly written based on the CDS software notes ${ }^{1}$, CDS data analysis lectures by Giulio Del Zanna ${ }^{2}$, and the content material of the header notes of the SolarSoft ${ }^{3}$ programs utilised in this work. The data reduction and analyses were carried out using the IDL programming language.

\subsubsection{Bias subtraction}

The first step after reading-in the VDS images (images taken by the NIS detector, known as Viewfinder Detector Subsystem VDS), is removing the read-out bias from the data. This is done by applying the VDS_DEBIAS routine. The NIS SYNOP study is designed such that it includes the four CCD quadrants (four background windows at the corners of the CCD). The quadrants have different offset values (see table 2 in CDS software note $\left.13^{4}\right)$. VDS_DEBIAS subtracts the average CCD bias as a function of the read-out quadrants. Before bias subtraction, the unit of the raw data is "ADC" (Analogue to Digital Conversion). This is changed to "Debiased-ADC" after applying VDS_DEBIAS.

\subsubsection{Removing cosmic ray hits}

The second step is to clean the data from cosmic spikes (the spike in the signal recorded by one or more pixels of the sensor when hit by a cosmic ray). The effect of cosmic ray hits on the CCD strongly constrains the instrument observations. The cosmic rays are a continuous flux of protons, $\alpha$ particles and heavy energetic ions originating from our galaxy. Although the flux rate is low, its continuity restricts the exposure time of the instrument.

There are a number of routines in SolarSoft library which can be used to detect and remove the cosmic spikes from the data. Among them all, the "CLEAN_SPIKE" and "NEW_SPIKE" routines, both written by Peter Young, are the more robust and reliable.

\footnotetext{
${ }^{1} \mathrm{http}: / /$ sohowww.nascom.nasa.gov/solarsoft/

${ }^{2}$ http://www.damtp.cam.ac.uk/user/astro/gd232/research/medoc_03/

${ }^{3}$ http://www.lmsal.com/solarsoft/

${ }^{4}$ http://solar.bnsc.rl.ac.uk/swnotes/cds_swnote_13.pdf
} 
The NEW_SPIKE routine is a revised version of the CLEAN_SPIKE and, although it follows an identical method to remove the cosmic spikes, it runs significantly quicker ${ }^{1}$. Both routines assume that cosmic rays are only confined to single exposures. In a given exposure, pixels are flagged as cosmic ray hits when their values are high compared to the neighbouring exposures. Suppose we have a 3D array with dimensions $X, Y$ and $Z$ (here $\lambda$, solar $y$ and solar $x$, respectively). Taking an X-Z plane as an exposure, the routines then scan along the $Y$ direction to find any pixel with a value greater than its immediate $Y$ neighbour by a certain amount named cutoff. In the NEW_SPIKE, the cutoff value is set to 0.65 . The flagging of the pixels with potential cosmic ray hits is fulfilled using the following criterion:

$$
\frac{P-A V_{y}}{A V_{y}}>C U T O F F
$$

where $P$ is the pixel value and $A V_{y}$ is the average of the immediate $Y$ neighbors. To avoid the removal of spikes in the background, a lower limit should also be defined below which the cosmic rays are regarded as noise and are not going to be flagged. The FMEDIAN routine estimates the local background $\left(\mathrm{BG}_{L}\right)$ around the pixel of interest. Now the pixel value to be flagged as cosmic ray must satisfy the following criterion as well:

$$
P-M>B G_{L}
$$

where $P$ is the pixel value and $M$ is the median. Other criteria are also applied in order not to flag the pixels with solar large values. These usually correspond to transient brightenings in transition region lines (for a more detailed explanation, the reader is referred to CDS software note $46^{2}$.) To confirm the spike identification, the same checks will be done by the routine in the other two dimensions and if at least one of these checks confirms the spike, then it will be marked as a cosmic spike. The affected pixels can be set to 'MISSING' (useful for analysis) or to the average of the two neighbouring X-pixels (useful when producing images). In most cases, the cosmic rays appear in a single peak. But there are also situations in which they have two neighbouring pixels with large values. In such cases, the pixel of interest is being checked with the two pixels adjacent to the neighbouring pixels. If the number of such double-peak spikes is not too large, the flagging can also be done manually. However, because of the very large dataset, the manual check was not carried on in this work.

\subsubsection{Detection of damaged spectra}

The NEW_SPIKE routine may encounter problems in distinguishing a cosmic spike when it is at or close to the peaks of the spectra. In addition, the fit to a given spectrum with either a cosmic spike at its peak or more than one cosmic spikes at other parts is associated with large uncertainty. Therefore, the spectra which were highly damaged due to cosmic

\footnotetext{
1 CDS Software note 46: Missing Pixels and Cosmic Rays

${ }^{2}$ http://solar.bnsc.rl.ac.uk/swnotes/cds_swnote_46.pdf
} 

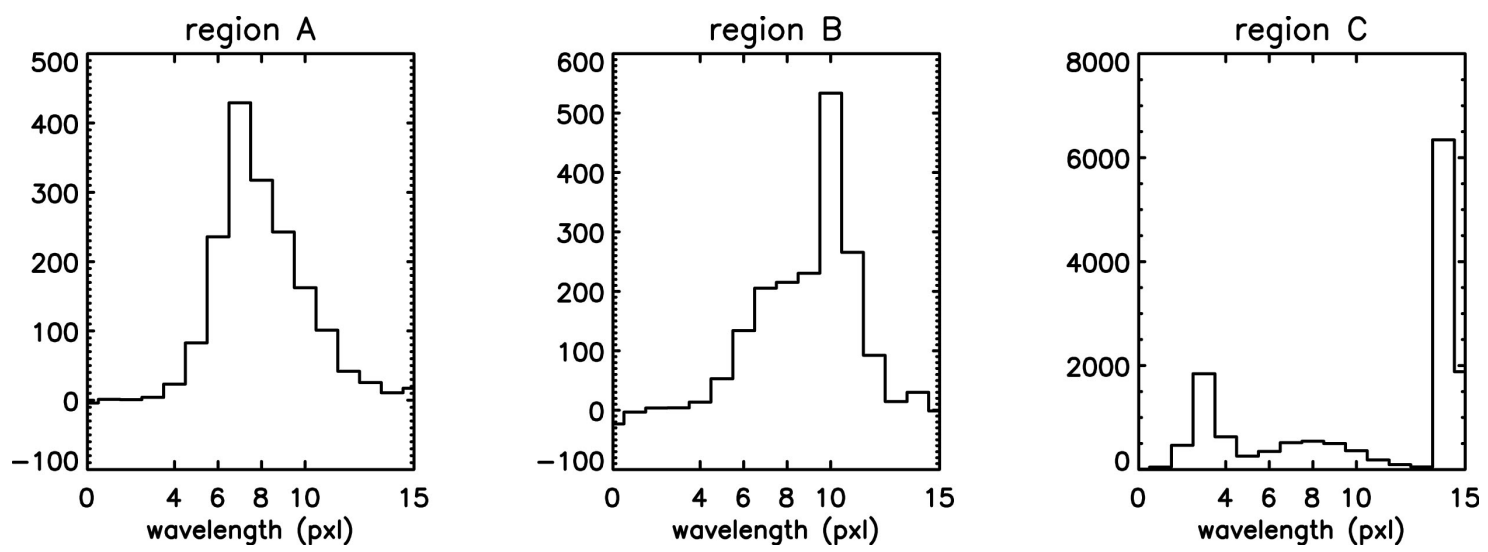

Figure 3.1: Examples of three flagged spectra. From left to right, the spectra having 1 or more than one cosmic ray hits on their regions $A, B$ and $C$, respectively (see text).

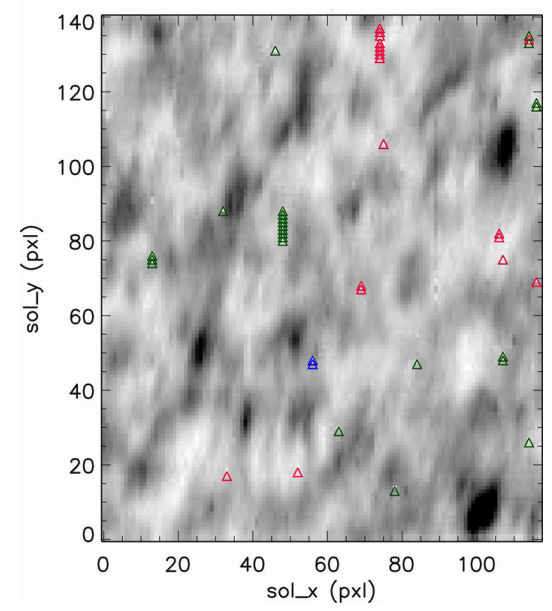

Figure 3.2: Flagged spectra over a given CDS raster scan, taken on 1996 April 28 in the $\mathrm{O} v$ window. The image is shown in a negative scale. The red, blue and green triangles indicate the positions of the flagged spectra, containing 1,2 and more than 2 cosmic ray hits at regions $A, B$ and $C$, respectively.

ray hits were totally dismissed from the analyses. For tracking these spectra, three regions were defined on the spectra:

Region $A$ : including wavelength pixels around line peak

Region $C$ : including wavelength pixels on the line wings

Region $B$ : including wavelength pixels between the peak and the wing regions

Examples of these three regions is shown in Fig. 3.1. The spectra with 1, 2 and more than 2 cosmic spikes in their regions $A, B$ and $C$, respectively, were considered as damaged spectra and flagged out from further analyses. Fig. 3.2 depicts the position of the flagged spectra on a given CDS raster scan. The red, blue and green triangles correspond to the positions of the spectra meeting the above mentioned criteria. On average, about $0.3 \%$ of the spectra were flagged in a typical raster scan.

The NEW_SPIKE routine returns the number of flagged pixels for each spectral window. In Fig. 3.3, the long-term variation of the number of affected pixels by cosmic 

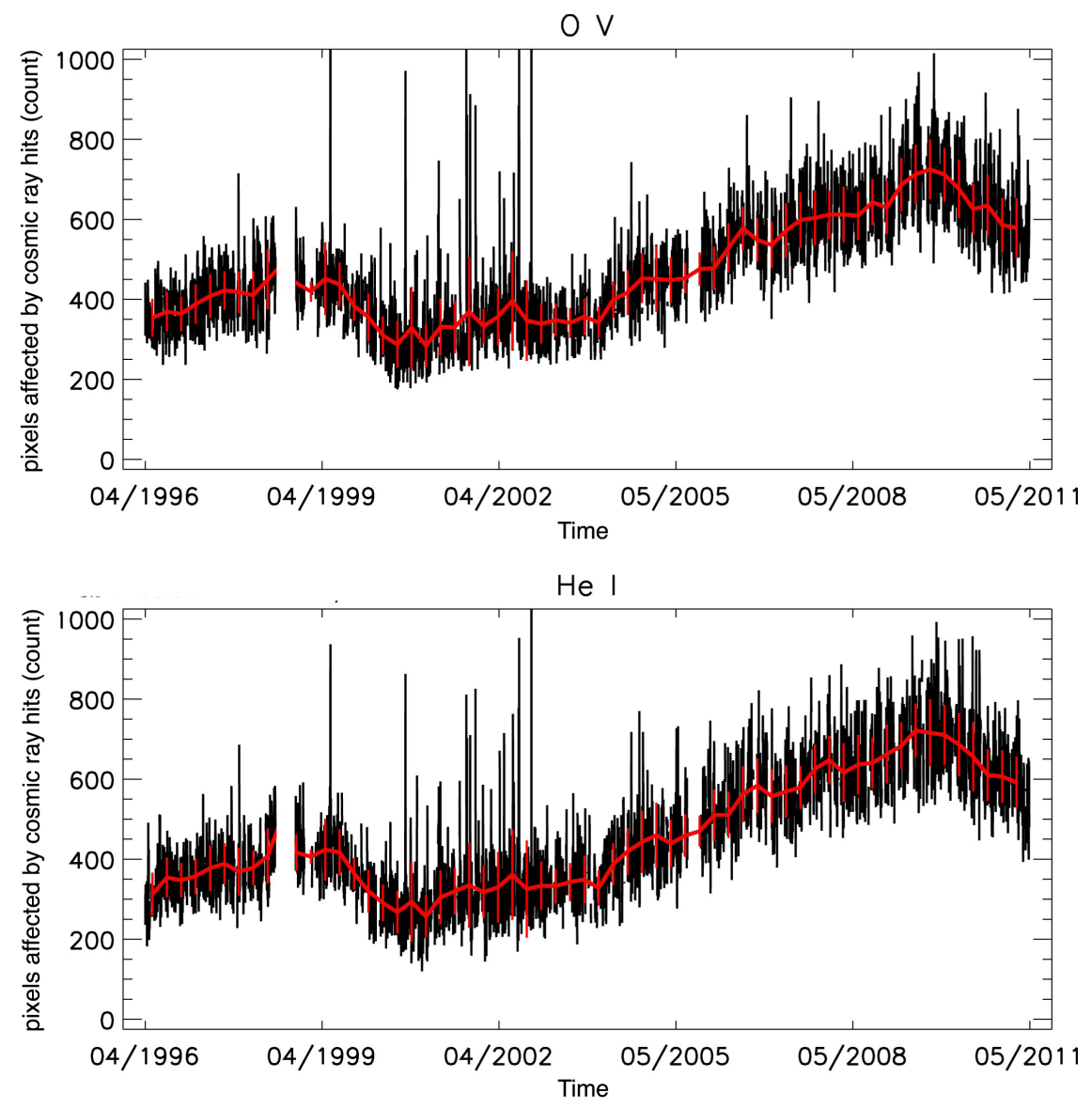

Figure 3.3: Number of affected pixels by cosmic ray hits in the $\mathrm{O} v$ (top) and He I (bottom) windows. The solid red line and its corresponding error bars represent 90 -day average values and their $1 \sigma$ standard deviation, respectively.

rays is illustrated. The black lines in the top and bottom panels correspond to the number of flagged pixels due to cosmic ray hits in the $\mathrm{O} v$ and $\mathrm{He}$ I windows, respectively. The red lines in both panels are the 90-day average values and their associated $1 \sigma$ standard deviation, with particularly high-counts occur mostly close to the maximum of the solar cycle 23 and are mainly due to sudden output of energetic particles from the Sun.

It is important to note here that the number of the affected pixels must not be read as the number of the cosmic ray hits, since individual hits can affect several pixels on the CCD. The plot only shows the modulation of the affected pixels over the course of the solar cycle 23. Translating the number of affected pixels into cosmic ray hits is a complicated procedure (Pike and Harrison 2000). Such an analysis was not carried out this thesis work. A theoretical overview of the cosmic rays and their modulation with solar activity is provided by Schrijver and Siscoe (2012) and Potgieter (2013).

\subsubsection{Image distortion and other effects}

NIS spectra suffer from geometrical distortions such as rotation and tilt of the spectra, resulting from the misalignments between the detector and the grating, and between 


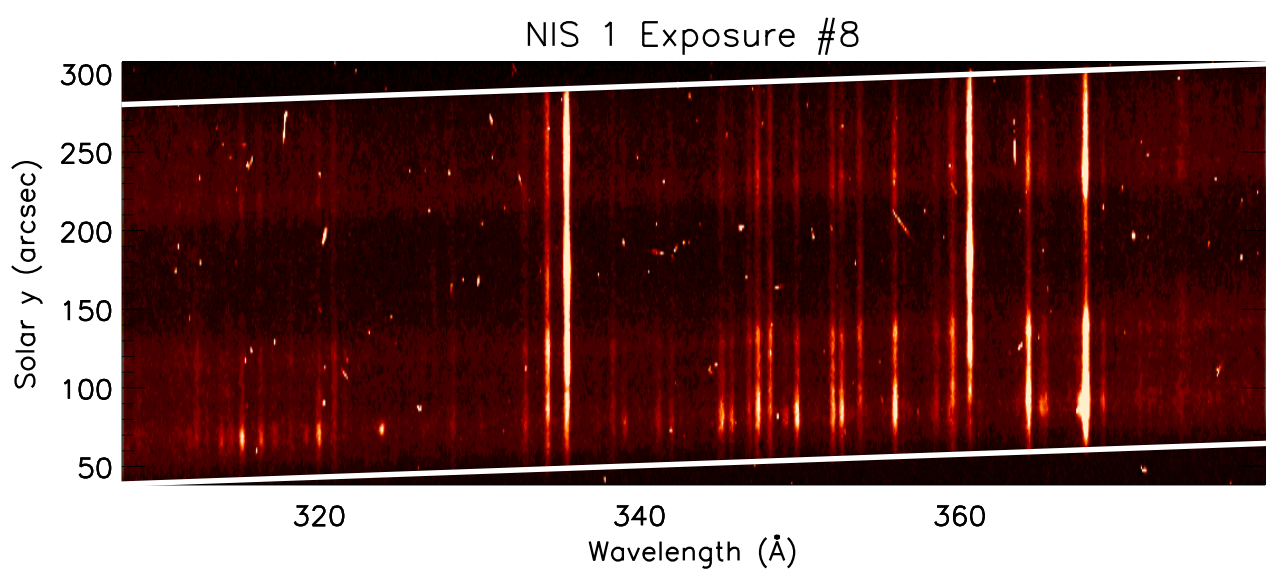

Figure 3.4: Geometric distortion in the NIS 1 spectral band in an exposure, taken on 1997 Sep 25. The slightly-tilted horizontal white lines show how the dispersion direction is rotated relative to the CDS detector. Also, the angles between the spectral lines and the dispersion direction deviate from the right angle, meaning that the spectral lines are tilted. The image also illustrates the effects of the cosmic ray hits.

the grating and the slit, respectively (Fig. 3.4). The rotation and tilt angles are slightly different for NIS-1 and NIS-2. Two standard routines are applied to correct these artefacts: VDS_ROTATE and NIS_ROTATE.

Besides these main artefacts, Del Zanna et al. (2001) discussed that the spectral lines observed with the $2^{\prime \prime}$ and 4 " slits have very similar line widths, almost 2 times larger than the measurements prior to the launch, and concluded that the NIS instrument is not in focus. In addition, the instruments slits are not co-aligned and, therefore, there is an offset in the detector $y$ in the images taken by different slits (Del Zanna 1999). There is no possible correction for these artefacts.

\subsubsection{Data calibration}

Due to being continuously exposed to solar radiation, the efficiency of the multichannel plates (MCPs) in the CDS detector decreases over time (Thompson 2000). This effect is known as burn-in and occurs mainly in the centres of the strongest lines. In addition, the sensitivity (or quantum efficiency) of individual pixels on the CCD is different, leading to a $2 \mathrm{D}$ pattern of the gains of the detector. The process of removing this pattern from the image is called flat-fielding. The burn-in and flat-field corrections of the CDS data are the main issues to be dealt with when calibrating the CDS data. These effects are explained in detail in CDS software notes $13^{1}, 34^{2}$ and $51^{3}$. These corrections are applied to the data using the standard routine VDS_CALIB.

Up to here, the unit of the data is DEBIASED-ADC. The VDS_CALIB, beside applying the above mentioned corrections, divides the data by the exposure time and returns the DEBIASED-ADC count rate. Dividing the data then by throughput value, the unit will be

\footnotetext{
${ }^{1}$ http://solar.bnsc.rl.ac.uk/swnotes/cds_swnote_13.pdf

2 http://solar.bnsc.rl.ac.uk/swnotes/cds_swnote_34.pdf

3 http://solar.bnsc.rl.ac.uk/swnotes/cds_swnote_51.pdf
} 
converted to photon - events/pixel/second. The detector's throughput is defined as the number of Analogue-to-Digital Conversion (ADC) for each EUV photon detected by the MCP (referred to as photon-event). Hence, the throughput is a measure of amplification of the detector and varies for different operating voltages. The operating voltage of the MCP in the majority of CDS observations including the SYNOP study is $756 \mathrm{~V}$. This corresponds to a throughput value of 2.27, meaning that each photon-event results in 2.27 $\mathrm{ADC}$ counts. The total number of $\mathrm{ADC}$ is proportional to the MCP quantum efficiency times the throughput value.

The last step is to convert the unit of the data from photon-events calculated by VDS_CALIB into physical units. This is a complex procedure and several factors have to be taken into account: the quantum efficiency of the detector, the NIS wavelength calibration (which varies with the slit used to take the raster scan) and the effective area of the CDS telescope and NIS detector. Regarding the wavelength calibration, CDS has not a real fixed wavelength range and there are small variations in the zero point of the pixel-wavelength relation. The reason is the temperature changes caused by the varying illumination of the NIS instrument.

These factors are applied to the data by NIS_CALIB. The routine returns the data in a default unit of photons $/ \mathrm{cm}^{2} / \mathrm{s} / \operatorname{arcsec}^{2}$. The returned unit can be modified by passing the following keywords:

ERGS: $\quad$ returns the data in units of ergs instead of photons.

STERADIANS: returns the data as photons $/ \mathrm{cm}^{2} / \mathrm{s} / \mathrm{str}$.

ANGSTROMS: returns the data as photons $/ \mathrm{cm}^{2} / \mathrm{s} / \operatorname{arcsec}^{2} / \AA$.

In this work, the keywords were set in a way to return the data with the unit of $\mathrm{erg} / \mathrm{cm}^{2} / \mathrm{sec} / \mathrm{steradian}$ or, equivalently, $\mathrm{mW} / \mathrm{m}^{2} /$ steradian.

\subsubsection{Least-square fitting of the spectra}

After applying the instrumental standard calibration and correction routines, the solar radiance at each spatial pixel is determined by least-squares-fitting of a single Gaussian function with constant background to a pre-loss spectrum, i.e. any spectrum recorded prior to 1998 Jun 25 , and by fitting a broadened Gaussian line profile to each post-loss spectrum.

After the recovery of the CDS on 1998 Aug 3, it turned out that the line profiles of the spectral lines in both spectral bands (NIS-1 and NIS-2) had been broadened (extended over 24 wavelength pixels, compared to 18 pixels prior to loss) and were asymmetric (with slightly larger values on the right (redward) wings), and it was not possible to model them with a single Gaussian any more. The CDS team then came up with a broadened-Gaussian line profile function to model the spectral lines and extract the radiance. The procedure is explained in the CDS Software Note $53^{1}$. Briefly, the model function $B(\lambda)$ is a combination of two terms, a Gaussian function $G(\lambda)$ and a term describing the wings of the line profiles $W(\lambda)$ :

$$
G(\lambda)=\exp \left[-\frac{1}{2}\left(\frac{\lambda-\lambda_{0}}{\sigma}\right)^{2}\right]
$$

\footnotetext{
${ }^{1}$ http://solar.bnsc.rl.ac.uk/swnotes/cds_swnote_53.pdf
} 

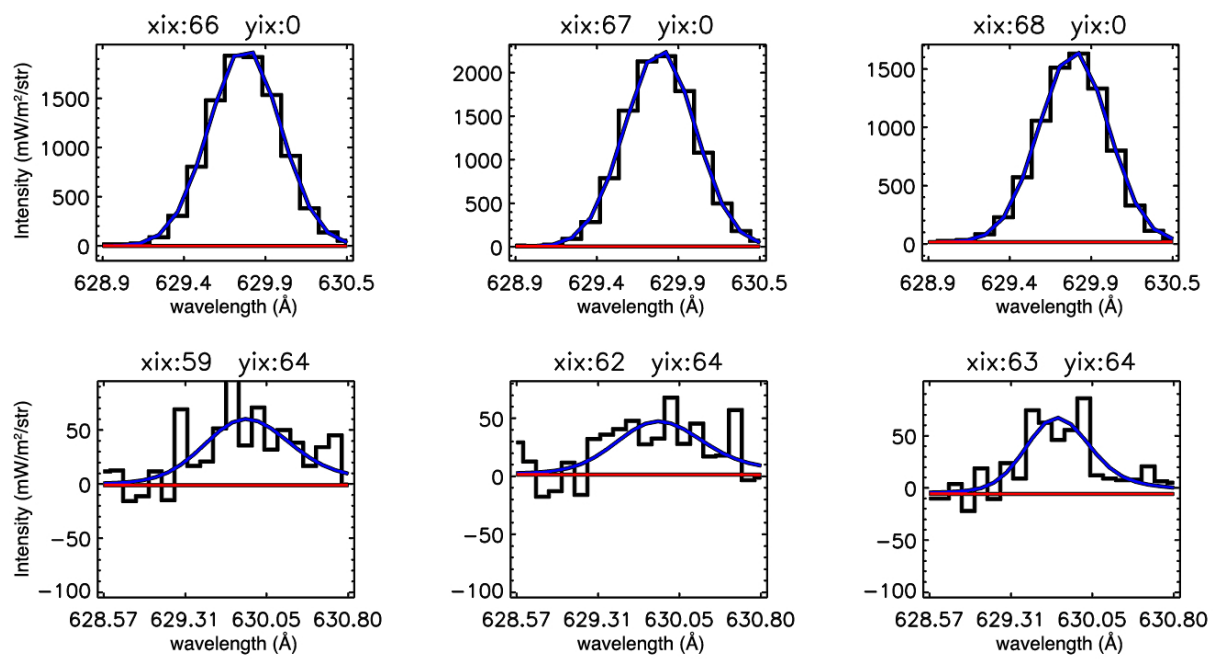

Figure 3.5: Examples of the spectra and their Gaussian fits in the darkest (bottom row) and brightest (top row) regions of a given CDS image. The blue and the red lines are the Gaussian fits and the constant fitting backgrounds, respectively.

$$
\begin{gathered}
W(\lambda)=\frac{1}{\left(\frac{\lambda-\lambda_{0}}{\sigma^{\prime}}\right)^{2}+1}, \\
B(\lambda)=A_{0}[(1-\alpha) G(\lambda)+\alpha W(\lambda),
\end{gathered}
$$

where $\alpha$ may have different values for the left and right wings of the spectral lines and $\sigma^{\prime}=2 \sigma \sqrt{2 \ln (2)}$. The line intensity after extracting the fitting parameters can be calculated using the following function:

$$
I=\sqrt{2 \pi} A_{0} A_{2}\left[\left(1-\frac{A_{3}}{2}\left(1+A_{4}\right)\right)+A_{3}\left(1+A_{4}\right) \sqrt{\pi \ln (2)}\right] .
$$

The parameters in the intensity equation are:

$$
\begin{array}{ll}
A_{0} & \text { the amplitude of the line profile, } \\
A_{1}=\lambda_{0} & \text { the line center position, } \\
A_{2}=\sigma & \text { the Gaussian width, } \\
A_{3}=\alpha_{\text {right }} & \text { the relative amplitude of the right wing, } \\
A_{4}=\alpha_{\text {left }} / \alpha_{\text {right }} & \text { the ratio of the amplitude of the left wing to the right wing. }
\end{array}
$$

The best results are achieved with $A_{3}=0.8$ and $A_{4}=1.0$ for NIS- 1 and $A_{3}=0.317$ and $A_{4}=0.297$ for NIS-2. The intensity of the line profiles before the loss of SOHO can be simply calculated using the values of the peak amplitude $A$ and width $\omega$ of the Gaussian fits.

$$
I=\sqrt{2 \pi} A \omega
$$




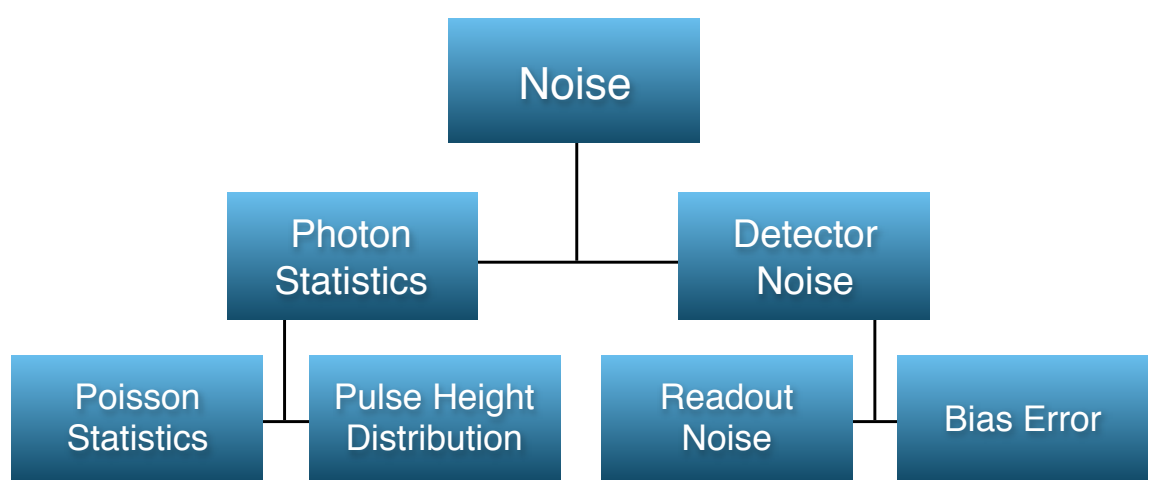

Figure 3.6: Various sources of noise in NIS data.

The fitting procedure was done using the IDL program Component Fitting System CFIT $^{1}$. The program needs special supervision in order to produce the best fits. The main issue is defining the initial fitting values and setting up lower and upper limits for different fit parameters. The program also returns the $1 \sigma$ standard deviation for each parameter. These will be later utilized to estimate the error in the total intensity.

It is important to note that for properly fitting the CDS data, they have to be weighted with $1 / \sigma^{2}$. The parameter $\sigma$ is the noise and is given by $\sigma=f_{N} S$, where $S$ is the signal and $f_{N}$ is the fractional noise. This will be discussed in the next section.

The quality of the fits at darker regions of the images are usually very poor. This, as will be shown in the next chapter, has a significant effect on the analysis carried out in this work. Fig. 3.5 depicts few examples of the Gaussian fitting of six spectra at brightest (top row) and darkest (bottom row) parts of a given raster scan, taken prior to loss of SOHO. The red lines are the constant backgrounds and the blue lines are the Gaussian fits. It is evident that the spectra in darker regions are heavily contaminated with noise. It will be shown that this leads to an underestimation of the radiance in the darker parts of the image.

The final output of the whole process is a set of daily intensity images, with the intensity at each pixel corresponding to the area under the fitting Gaussian after subtracting the background.

\subsubsection{Noise propagation}

There are two main sources of noise in NIS data: the photon statistics, which is the dominant source, and the detector noise. The latter is due to the fact that VDS is not a photon-count detector, and the conversion from ADC unit to photon-events applies extra noise to the data. The contributions to the total noise is schematically depicted in Fig. 3.6 and are discussed in the following. The detailed information on deriving statistics from NIS data can be found in CDS Software Note $49^{2}$.

\footnotetext{
1 http://solar.bnsc.rl.ac.uk/swnotes/cds_swnote_47.pdf

${ }^{2}$ http://solar.bnsc.rl.ac.uk/swnotes/cds_swnote_49.pdf
} 


\subsubsection{Photon noise}

The photons striking the CCD of the detector follow the Poisson distribution and their statistics is associated with the Poisson noise. For a given number of photon-events in a pixel, the corresponding Poisson noise is the square-root of the photon-event counts. Hence, the first step in calculating the Poisson noise is converting the raw data into photon events/pixel/s. This can be done by applying the VDS_CALIB routine, as explained in Sec. 3.3.5. Multiplying the output with the exposure time returns the photon-events / pixel, or namely $N$, with associated Poisson noise of $\sqrt{N}$.

Another source of noise lies in the process of photon detection itself. The arrival of a photon on the photocathaode of a photomultiplier tube results in the emission of photoelectrons, which on their way toward the anode, get multiplied by electron multipliers known as dynodes, through the process of the "secondary emission" (see chapter 6.1 in HamamatsuPhotonics. 2007). The strength or height of the output pulse produced by each photon-event, therefore, depends on the rate of electron multiplication by the dynodes. Note that the electrons produced inside the photomultiplier travel through different paths and not all of them interact with all succesive dynodes. The number of secondary electrons produced for each primary electron has a broad probability, similar to the Poisson distribution. Consequently, the pulses obtained at the anode of the photomultiplier have various heights, with a distribution that can be seen as a Poisson distribution. This is know as Pulse Height Distribution $P H D$ and applies additional noise to the data, comparable to the Poisson noise of $\sqrt{N}$.

The total photon noise is calculated as the square root of the sum of the squares of the Poisson noise and the PHD:

$$
\sigma_{\text {photon }}=\sqrt{\sigma_{\text {Poisson }}^{2}+\sigma_{P H D}^{2}}=\sqrt{2 N} .
$$

\subsubsection{Detector noise}

The process of reading out the data applies additional noise (to the data), especially when the photon count rates are low. This is attributed to imperfect detector electronics and to the electromagnetic environment that surrounds it (see section 5.1 in CDS software note $13^{1}$ ). The NIS RMS deviation ( $1 \sigma$ readout noise) as measured prior to the launch of the instrument ranges approximately between 1.5 and $2 \mathrm{ADC} /$ pixel. By dividing these values by the detector throughput (2.27, see Sec. 3.3.5), one can convert ADC to photon-events/pixel. This will return the average readout noise $\sigma_{R}$ of about 0.7 photon-events/pixel. Conservatively, an upper limit of 1 photon-events/pixel is usually assumed as the readout noise. The average readout noise of a group of $n$ pixels is $\sqrt{n} \sigma_{R}$.

There is an additional error, about 1 in ADC units ( 0.5 photon - events/pixel), associated with the de-biasing of the data. This is small compared to the scattered light in the instrument, mainly from Lyman- $\alpha$ and the Lyman continuum. This is usually taken into account, together with the background uncertainty in the process of the Gaussian fitting, and contributes to the error associated with the intensity values.

${ }^{1}$ http://solar.bnsc.rl.ac.uk/swnotes/cds_swnote_13.pdf 


\subsubsection{Total noise}

The two main sources of noise mentioned above are independent. Hence, the total noise can be calculated by:

$$
\sigma_{\text {total }}=\sqrt{\sigma_{\text {photon }}^{2}+n \sigma_{R}^{2}}
$$

where $\sigma_{\text {photon }}$ is the total photon noise, $\sigma_{R}$ is the readout noise, and $n$ is the number of pixels being summed together. This will give the $\sigma_{\text {total }}$ in units of photon - events / pixel. However, the NIS data can be expressed in different units and it is practical to define a unitless fractional noise $f_{N}$ as the ratio of the total noise to the signal:

$$
f_{N}=\frac{\sigma_{\text {total }}}{N}=\frac{\sqrt{2 N+n \sigma_{R}^{2}}}{N} .
$$

Multiplying the fractional noise $f_{N}$ by the calibrated data (in any unit) returns the total statistical noise $\sigma$, which is used to weight the data prior to the Gaussian fitting procedure.

\subsubsection{Error in the intensity}

The estimation of the total noise is crucial in terms of having the best Gaussian fits to the spectra and calculating the intensity based on fit parameters (Sec. 3.3.6). The error estimation is calculated by:

$$
\left(\frac{\sigma_{I}}{I}\right)^{2}=\left(\frac{\sigma_{A}}{A}\right)^{2}+\left(\frac{\sigma_{\omega}}{\omega}\right)^{2}
$$

where $I$ is the intensity, $A$ and $\omega$ are the amplitude and the width of the fit, respectively, and $\sigma_{I}, \sigma_{A}$ and $\sigma_{\omega}$ are their corresponding $1 \sigma$ standard deviation. This is valid only if the amplitude and the width of the Gaussian fit are considered to be completely independent. On the other hand, if these two are correlated, this will change the error estimation to:

$$
\left(\frac{\sigma_{I}}{I}\right)^{2}=\frac{1}{2}\left[\left(\frac{\sigma_{A}}{A}\right)^{2}+\left(\frac{\sigma_{\omega}}{\omega}\right)^{2}\right],
$$

as stated in the CDS software note $13^{1}$. A Monte Carlo simulations showed that the second approach is more favourable, in the sense that it comes closer to describing the actual data.

\subsection{CDS point-spread function and data binning}

The point-spread function (PSF) defines how a point source appears when imaged by the instrument. Large PSF simply means that the adjacent pixels on the CCD are not independent in their brightness. The full-width-at-half-maximum $(F W H M)$ of the PSF is a measure of the spatial resolution of the instrument.

\footnotetext{
${ }^{1}$ http://solar.bnsc.rl.ac.uk/swnotes/cds_swnote_13.pdf
} 
By an intercalibration of the SUMER detector A and the CDS/NIS, Pauluhn et al. (1999) pointed out that CDS has a broad PSF corresponding to $F W H M_{x}=6^{\prime \prime}$ and $F W H M_{y}$ $=8^{\prime \prime}$, which is almost 3 times larger than the pixel size of the NIS detector in the $x$ direction and over 4 times larger in the $y$ direction. This study was based on observations prior to the SOHO loss of control and the post-recovery data are believed to have an even larger point-spread function. Given the broad PSF, binning the intensity images sounds logical, since it increases the signal to noise ratio without reducing the spatial resolution.

The NIS data were binned with six different binsize values: from a binsize equal to 2 (binning over groups of $2 \times 2$ pixels) up to binsize equal to 7 (binning over groups of $7 \times 7$ pixels). The down-sampling (binning) of the data was carried out after applying instrument calibration corrections and prior to the spectra fitting. In this way, seven data sets corresponding to the different binsize values were prepared. The binsize 1 in the plots in the next chapter, means that the images have not been down-sampled and are in the original format.

\subsection{Data exclusion}

As mentioned earlier in the introduction, a selection process was carried out to exclude the rasters scans containing any structure deemed to be unusually bright for the quiet Sun (e.g. due to decaying ARs). The selection was done in two ways: firstly, by visual inspection of intensity images in the He I and O v lines and by referring to the He II $304 \AA$ images of the Extreme-Ultraviolet Imaging Telescope (EIT) (Delaboudinière et al. 1995) and the Michelson Doppler Imager (MDI) (Scherrer et al. 1995) level 1.8 full-disc magnetograms of the synoptic 96-minute series, and secondly, by analysing the goodness of the lognormal fits to the intensity distributions. This method will be explained in more details in Sec. 4.3.

For visual inspection, the EIT intensity images and MDI magnetograms were cropped in order to be consistent to the spatial coverage of the CDS images. In doing this, the time differences between the observation times of CDS raster scans and EIT and MDI data were considered in finding the areas corresponding to the CDS field of view (by taking into account the effect of the solar rotation).

Fig. 3.7 illustrates four images from the three instruments. The two upper CDS images show a small active region (AR) in the upper right corner. The same feature is also visible in the MDI and EIT images. Simultaneous comparison of the four images for each day makes is possible to select the raster scans containing bright structures with higher certainty. 

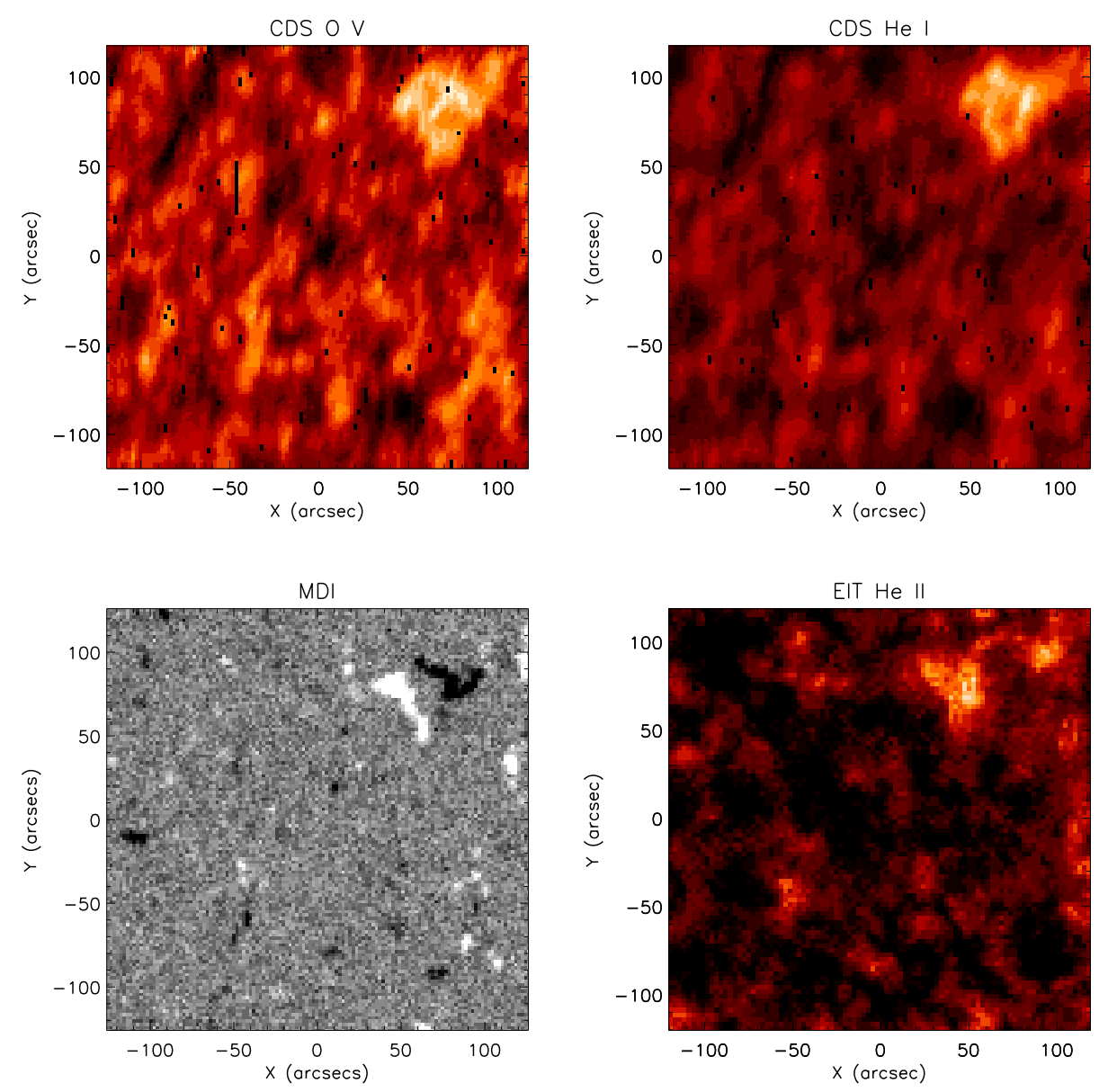

Figure 3.7: Appearance of a bright structure in the field of view of the CDS. The MDI magnetograms and EIT images were cropped in order to cover the same area of the solar surface as visible in the CDS images. 



\section{Analyses and results*}

The long-term variations of the radiance distribution in the chromosphere (He I $58.4 \mathrm{~nm}$ ) and in the transition Region $(\mathrm{O} \vee 63.0 \mathrm{~nm})$ of the quiet Sun were analysed taking two different approaches. First, by studying the daily average radiance over time and second, by investigating the intensity distributions. While, as it will be shown in the next section, the former approach posses very high uncertainty due to the degradation of the instrument, the latter provides more reliable results.

\subsection{Daily average intensities}

The CDS/NIS instrument, as common for EUV instrumentation, suffers from long-term degradation and decrease in responsivity. To verify the drop in the instrument responsivity, the data were read in and averaged over spatial and spectral dimensions, after removing the CCD read-out bias and before applying further calibrations. Panel $a$ ) in Fig. 4.1 displays
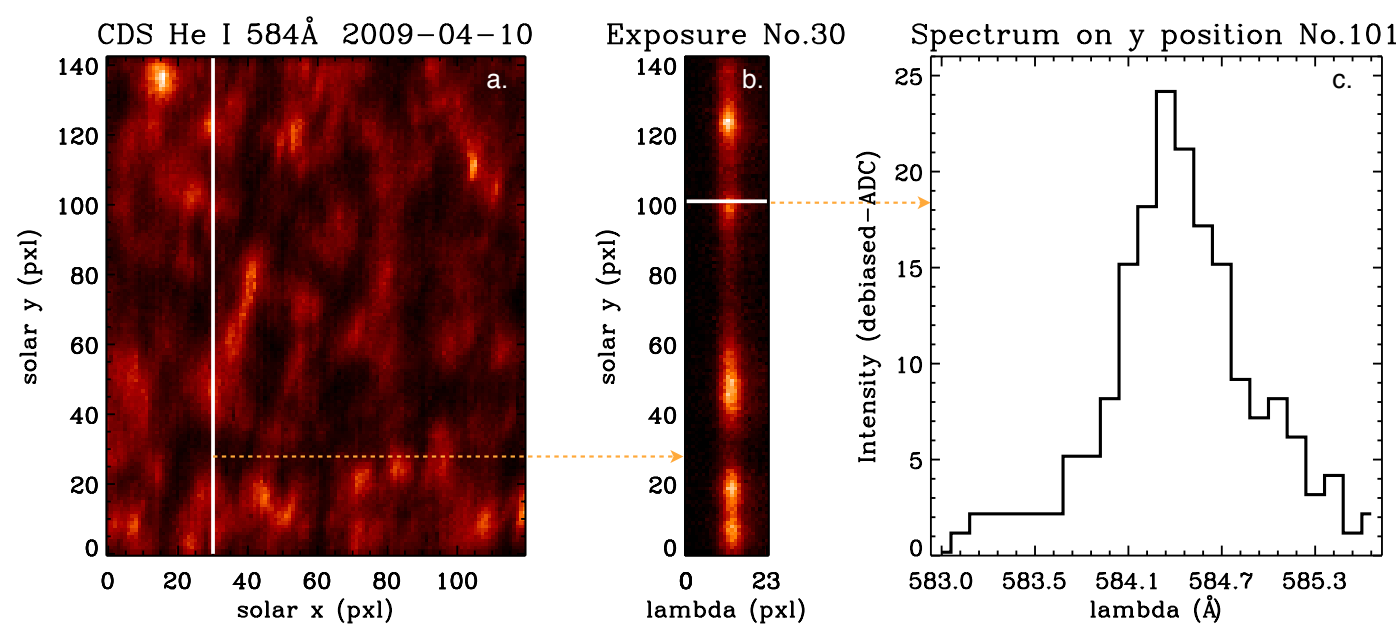

Figure 4.1: Panel $a$ ) intensity image in the He I spectral line. Each vertical column in the image corresponds to the average of the spectra over the wavelength dimension for a given $x$ position (i.e., exposure). Panel $b$ ) given exposure, corresponding to the white line in panel $a$ ), consisting of 143 spectra along the $y$ direction. Panel $c$ ) spectrum recorded along the horizontal white line in Panel $b$ ).

\footnotetext{
* A majority of the contents of this chapter and chapter 5 , has been already published as a journal paper by the author (Shakeri et al. 2015).
} 


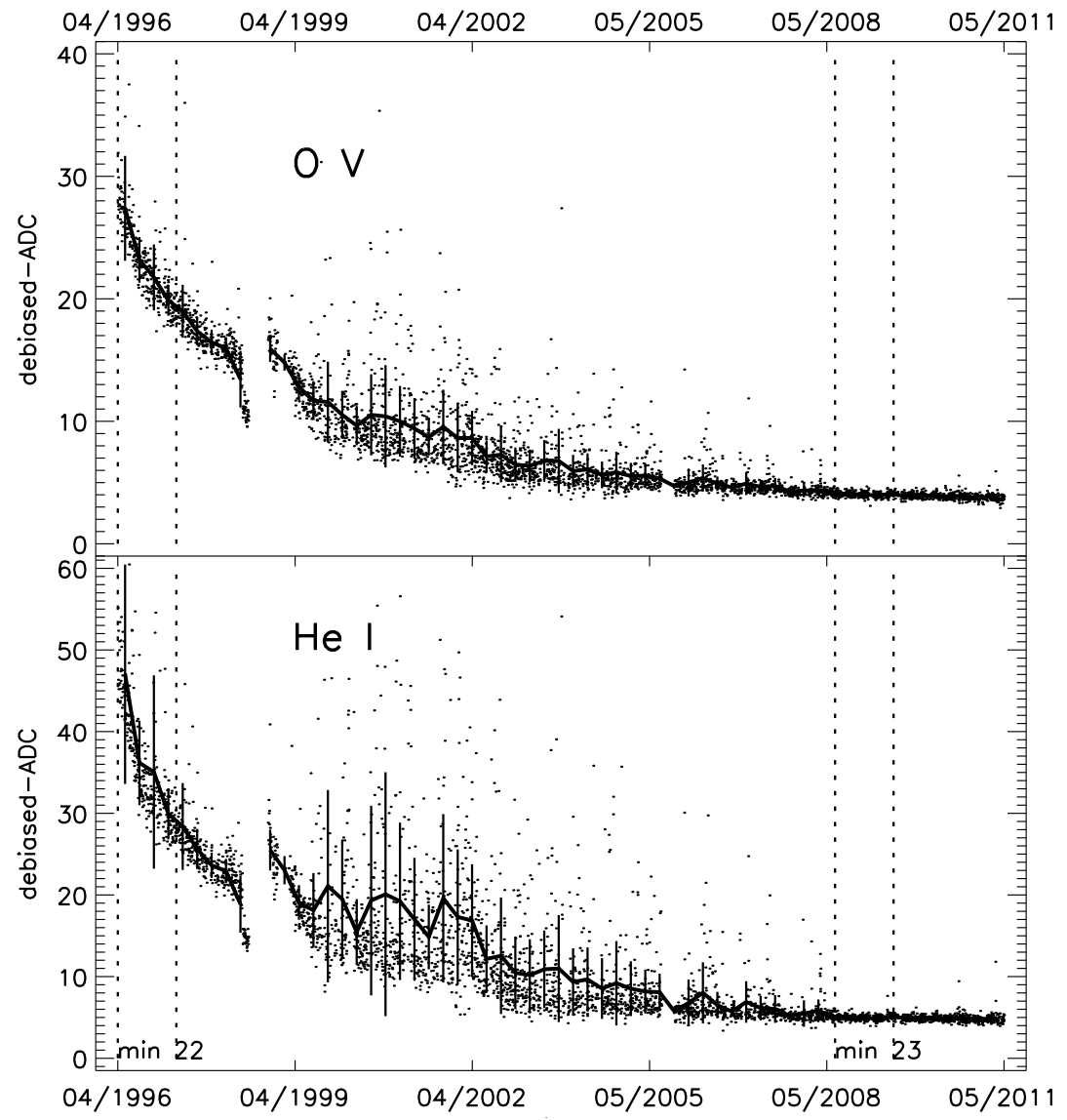

Figure 4.2: Illustration of long-term drop in CDS/NIS responsivity in the O V (top) and He I (bottom) spectral lines. The CCD readout bias has been removed from the data right after reading them in. No other calibration has been applied to the data. The solid lines and their associated error bars represent, respectively, the mean and the standard deviation, respectively, of the average intensity values over 90-day time intervals. The vertical dashed-lines indicate the two 1-year time spans used to compare the two solar activity minima.

an intensity image in the He I spectral line in debiased ADC units, i.e., after debiasing and prior to applying calibrations. Each $x$ position on the image corresponds to the average of an exposure over the $\lambda$ dimension. Panel $b$ ) shows a given exposure corresponding to $x$ position number 30 of the intensity image (vertical white line in Panel $a$ )). Panel $c$ ) depicts a spectrum recorded along the horizontal white line in panel $b$ ) at $y$ position number 101 , meaning that the intensity value given to the pixel coordinate $[30,101]$ in panel $a$ ) is the average of the spectrum in panel $c$ ). The daily average intensities with the units of "debiased ADC" were calculated by averaging over $x, y$ and $\lambda$ dimensions. These values are illustrated in Fig. 4.2, revealing a drastic drop in the responsivity of the instrument over the years. To compensate for this systematic drop, a set of calibration and correction routines has been produced and updated by the CDS team.

After applying standard calibrations to the data, the daily average intensities in physical units were calculated by fitting the spectra (as explained in Sec. 3.3.6) and averaging the derived intensities in the spectral line over the entire intensity images. The solid grey 


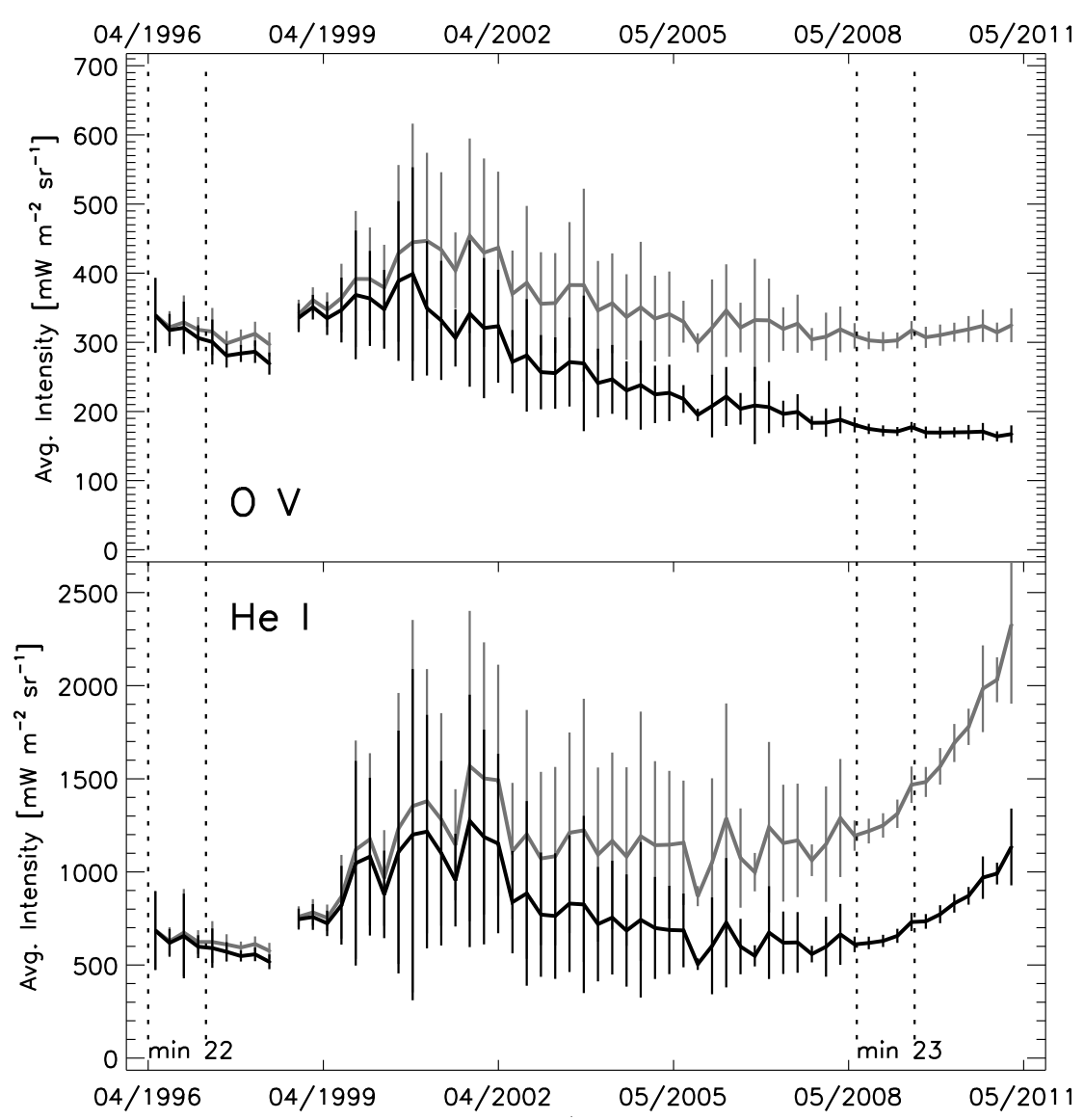

Figure 4.3: Long-term variation in daily average intensities of the $\mathrm{O} v$ (top) and $\mathrm{He} \mathrm{I}$ (bottom) spectral lines. The solid lines and their associated error bars represent the mean and the standard deviation of the average intensity values over 90-day time intervals before (grey) and after (black) applying the Del Zanna et al. correction factors (Del Zanna et al. 2010). The vertical dashed-lines indicate the two 1-year time spans chosen to cover the solar activity minima.

lines in Fig. 4.3 illustrate the long-term variations in the average intensities of the He I (bottom) and $\mathrm{O} v$ (top) spectral lines after applying standard calibrations. It represents the mean intensity values over 90-day time intervals, while the vertical bars represent its corresponding standard deviation. Del Zanna et al. (2010) suggested that the instrument standard calibration routines overcorrect the long-term drop in the responsivity of the instrument, resulting in a steady increase in the calculated radiance. By analysing the NIS daily synoptic observations from 1996 to 2009, they introduced a new set of correction factors to fix this issue. The black solid line and its corresponding error bars in Fig. 4.3 display the 90-day averaged intensities after applying the Del Zanna et al. (2010) correction factors.

The average He I intensity values calculated by using only the standard corrections (grey lines in Fig. 4.3) show an increase of intensity starting approximately from 2005. Such a trend is not seen in the $\mathrm{O} v$ line. Applying Del Zanna et al. correction factors (black lines) moderates the issue to a great extent for $\mathrm{He} \mathrm{I}$ but then the $\mathrm{O} v$ intensity values seem to be overcorrected for the period after 2008 . 


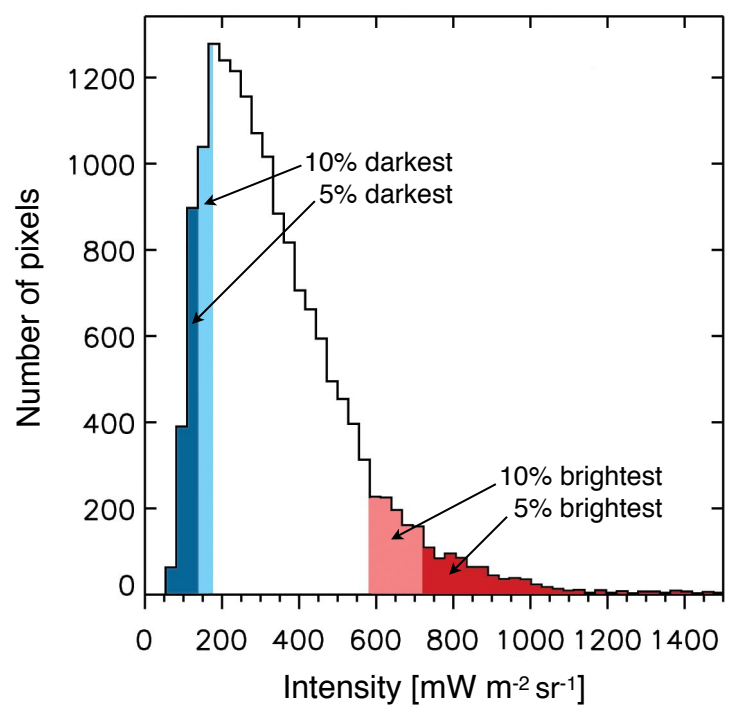

Figure 4.4: Histogram showing the number of pixels displaying intensity within a certain range on 1996 June 6 . The 5\% pixels with the lowest intensity are shaded dark blue. These, plus the shaded light blue pixels represent the $10 \%$ darkest pixels. Similarly the more strongly shaded dark red pixels represent the $5 \%$ brightest pixels. The $10 \%$ brightest pixels are the sum of the pixels shaded in dark and light red.

The correction factors introduced by Del Zanna et al. have been determined based on the assumption that the variations in the radiances of the chromospheric and transition region spectral lines are mainly due to the existence of the ARs and that the mean radiances of the spectral lines in the quiet Sun at disc-center essentially does not respond to the solar activity cycle. Although it is expected the solar radiance to be of the same order of magnitude at different solar minima, this assumption essentially precludes any investigation such as the present one, which aims at detecting even small variations in the quiet Sun's radiance, as long as they are significant. The small differences in radiance properties of the quiet Sun between two solar minima can be revealed only if the solar activity parameter under investigation is adequately independent of instrumental degradation. The average intensity values do not seem to satisfy this pre-requisite. The alternative is to identify and study parameters of the intensity distribution which are not affected by instrumental degradation. This is the approach that was decided to follow.

\subsection{Contrast ratios}

In order to minimize the effects of the calibration factors in the analysis, two ratios were defined, the 5 and 10 percent contrast ratios (called 5\% and 10\% CR from now on), defined as the ratios between the average intensities of the brightest $5 \%$ and $10 \%$ pixels within an intensity map and the average intensities of the darkest $5 \%$ and $10 \%$ pixels, respectively. Fig 4.4 displays a histogram corresponding to a given intensity map. The brightest and darkest pixels are highlighted in red and blue, respectively. The advantage of defining these CRs is that the correction factors, which have already been applied to the data, will be cancelled out by dividing. From now on the analysis are confined to the solar minima. 

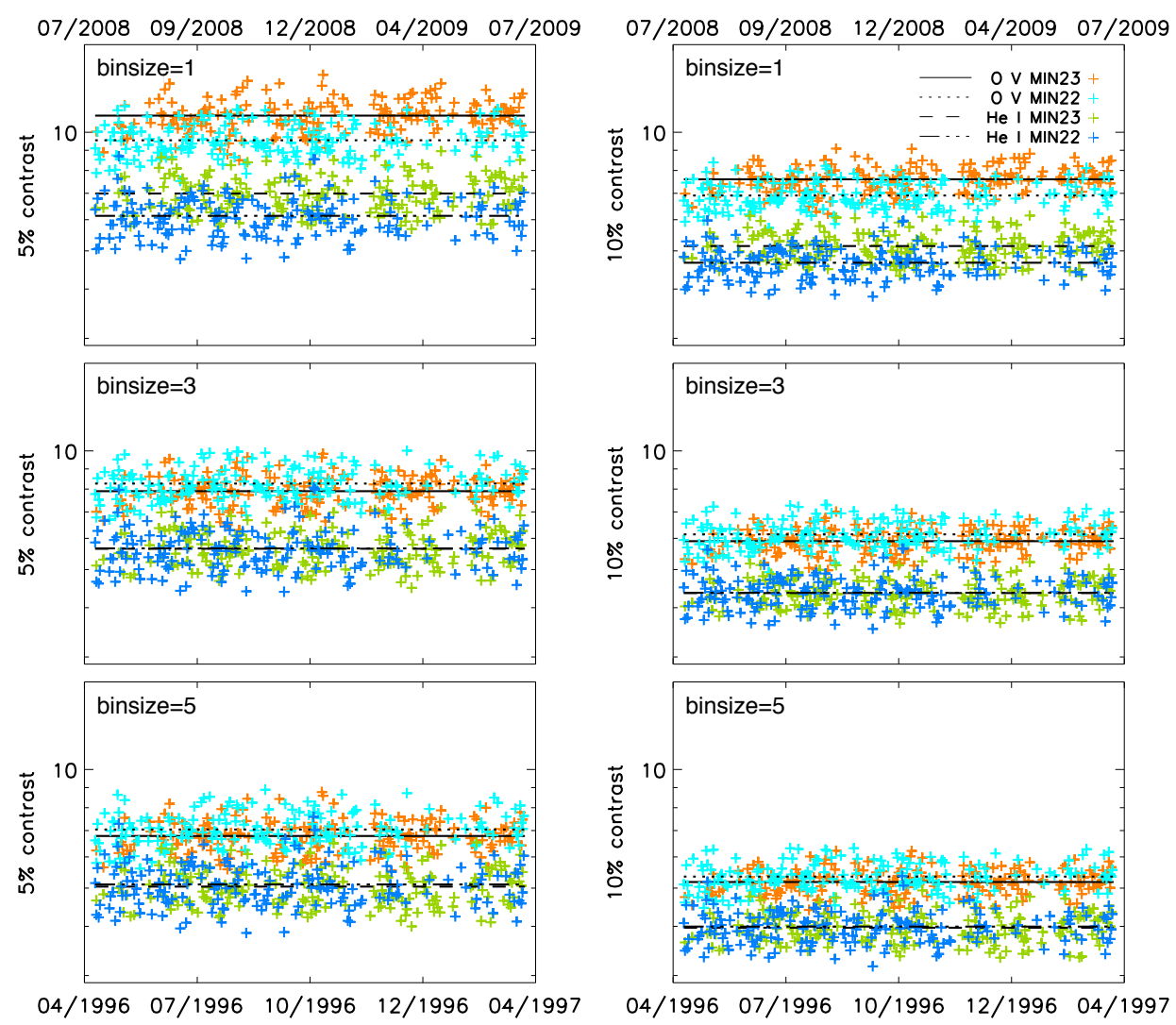

Figure 4.5: The 5\% (left column) and 10\% (right column) Contrast Ratios (CR) vs. time. See the main text for the definition and Fig 4.4 for an illustration. The 1-year periods during minimum 22 and minimum 23 are given at the bottom and top axis, respectively. Each panel represents results for a different binsize (labeled in the panel). The horizontal lines show the average value of each contrast ratio. Binsize 1 means that the intensity images are not binned. The solid and the dotted lines, correspond to the average of the $\mathrm{CRs}$ in the $\mathrm{O} v$ line (shown by orange and cyan plus signs) during solar minima 23 and 22, respectively. Similarly, the dashed and dashed-dotted lines show the mean values of the CRs in the He I line (indicated by the green and blue plus signs) over solar minima 23 and 22 , respectively. The $y$ axes are in logarithmic scale.

Fig. 4.5 illustrates the CRs for three different binsizes (see Sec. 3.4). In each panel of the figure the four colours distinguish between CRs of the He I and $\mathrm{O} v$ spectral lines at each minimum. The $x$ axes (of the bottom and top of each column of the figure) represent the date during solar minima 22 and 23, respectively. Excluded from the final plotted dataset, are days on which the CDS images sampled not purely quiet Sun (see Fig. 3.7) and/or because substantial parts of the image were missing.

The 5\% CRs are always larger than the $10 \%$ CRs. This is not surprising, since averaging over a larger number of pixels (10\% vs. $5 \%)$ at the bright and dark extremes of the intensity distribution results in closer mean values and consequently a closer-to-unity ratio. In addition, the CRs decrease as the binsize increases. This is also expected due to the fact that the intensity images tend to reduce towards "grey images" as more pixels are binned together. It is also evident that for all binsizes the $\mathrm{O} v$ line shows higher CRs than the 


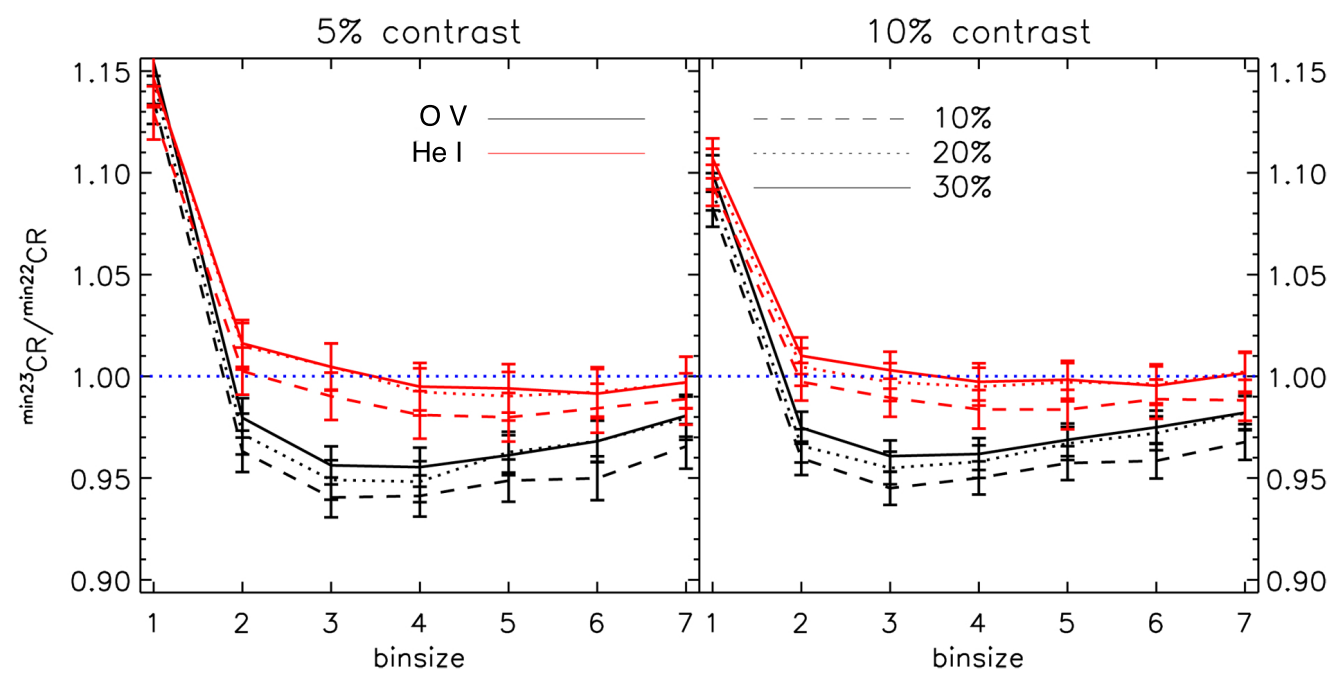

Figure 4.6: Ratio between contrast ratios at solar minima 22 and 23 for different binsizes. The blue horizontal dotted line indicates the unity line. The dashed, dotted and solid lines correspond to the three values of data exclusion, corresponding to $10 \%, 20 \%$ and $30 \%$ of images excluded from the dataset due to the existence of bright features inside the field of view, according to progressively stricter criteria. The error bars represent the $1 \sigma$ error in the ratios.

He I line. This difference starts from approximately 50\% higher CR values for binsize 1 , and drops as the binsize increases. The possible reasons for these differences will be discussed in detail in Sec. 5. What is more in focus here are the differences in CRs of the same spectral line over the two solar minima, e.g., differences between the solid and dotted horizontal lines (for $\mathrm{O} v$ ) or between the dashed and dash-dotted horizontal lines (for $\mathrm{He} \mathrm{I}$ ). This difference is quite large for both spectral lines when the intensity maps are not binned (top panels). However with increasing binsizes, it diminishes in the $\mathrm{He} \mathrm{I}$ line, while it persists in the $\mathrm{O} v$ line.

To have a direct comparison between the two minima, the ratio of the mean $\mathrm{CR}$ during solar minimum 23 to that during solar minimum $22\left({ }^{\min 23} \overline{C R} /{ }^{\min 22} \overline{C R}\right)$ for both spectral lines were computed. This ratio of CRs is illustrated in Fig. 4.6 as a function of binsize. The ratios are either close to or below the unity-line for all binsizes except binsize 1 . This is explained in details in Sec. 4.2.1.

In addition to the binsize values, the plots in the Fig. 4.6 also highlight the effect of data exclusion. Three different versions of data exclusion were applied to examine how sensitive the ratios are to the data exclusion. In version 1, almost $10 \%$ of the images (those containing the most obvious bright structures - visually chosen) were excluded from the data set. Versions 2 and 3 correspond to $20 \%$ and $30 \%$ of the data excluded from the dataset, respectively. They represent progressively stricter rejection criteria. It is evident from the plots that by excluding $20 \%$ of the data, the He I line has already reached a stable state since the ratios for versions 2 and 3 almost overlap and excluding more data points does not change the minimum-to-minimum ratios for any binsize. This is different for $\mathrm{O} v$ line, where excluding more data keeps pushing the ratios toward the unity line.

The plots also show that the minimum-to-minimum ratios are less than 1 for all binsizes (except binsize 1) in case of the $\mathrm{O} v$ line and are very close to unity in the He $\mathrm{I}$ line. This 
suggests that the intensity distribution of the $\mathrm{O} v$ line underwent significant changes between the last two solar minima while that of the upper chromospheric He I line did not.

\subsubsection{Peculiar case of binsize 1}

As it is shown in Figs. 4.5 and 4.6, the CRs prior to binning tend to be relatively larger in minimum 23 than in minimum 22. This is related to the fact that the darker pixels are dominated by noise for the data taken during solar minimum 23, due to the drop in the instrument responsivity (see Fig. 4.2). Hence, when making Gaussian fits to individual spectra in darker areas, the signal to noise ratio is so low that often what is being fitted is noise rather than the spectrum. Therefore, the line intensities in these areas tend to be underestimated. This effect is large for the data taken during minimum 23 and explains why the CRs are larger during solar minimum 23.

In order to see how the noise level affects the spectra over minimum 23, the intensity values of the pixels located at the wings of the spectra (in ADC unit, i.e., prior to debiasing) were compared to the NIS bias levels during the two minima. Three average values were determined to be compared to the bias readout from quadrants $\mathrm{C}$ and $\mathrm{D}$ of the NIS detector (the average is subtracted from the NIS2 data while debiasing). This comparison is illustrated in Fig. 4.7.

The three average values are: 1 . the mean of the 2 most external pixels on the wings of the spectra (i.e., pixels 0 and 17 prior to SOHO loss, and pixels 0 and 23 afterwards) marked by cyan diamonds in Fig. $4.7 ; 2$. the mean of the 4 most external pixels on the wings (i.e., pixels 0,1,16 and 17 of spectra prior to SOHO loss, and pixels 0,1,22 and 23 afterwards) marked by blue diamonds; 3 . the mean of pixels 2 and 15 of spectra prior to SOHO loss (pixels 2 and 21 afterwards) indicated by orange diamonds (internal pixels, hereafter). The black lines and the green vertical bars are the daily average bias levels and their associated $1 \sigma$ standard deviations, respectively, read by CCD quadrants $\mathrm{C}$ and $\mathrm{D}$. Panels $a$ ) and $b$ ) in the figure depict the already defined mean values for the $\mathrm{O} v$ (left) and He I (right) spectral lines, both calculated during minimum 22. The red panels at the top of the figure are the zoom-in views of the indicated frames on panels $a$ ) and $b$ ). In panels $c$ ) and $d$ ), the average values are shown for minimum 23 .

Panels $c$ ) and $d$ ) clearly show that the mean values of the 2 and 4 wing-pixels (cyan and blue diamonds, respectively) are within the error bars of the bias levels during minimum 23 , meaning that a considerable portion of each spectrum, on average, is below the noise level of the instrument. It is only the mean values of the internal pixels (orange) that are touching the upper limit (for the Ov line) or well above the bias error bars (for the He I line). This is not the case for minimum 22, where even the mean values of the 2 wing-pixels (cyan diamonds) are in most cases above the bias error bars.

An analysis of the background level of the spectra (which is a free parameter when fitting the spectra with Gaussian and broadened-Gaussian functions, see Sec. 3.3.6) shows that the fitted backgrounds during solar minimum 23 are significantly lower than those during minimum 23. Fig. 4.8 illustrates the difference between the average background levels of the He I spectra during the two minima for binsize 1 (top panels) and binsize 7 (bottom panels). The spectra were divided into two groups: the bright (right panels) and the dark (left panels) spectra, corresponding to the average of the 5\% brightest and 5\% darkest spectra of each intensity image, respectively. The plots show that the average background 

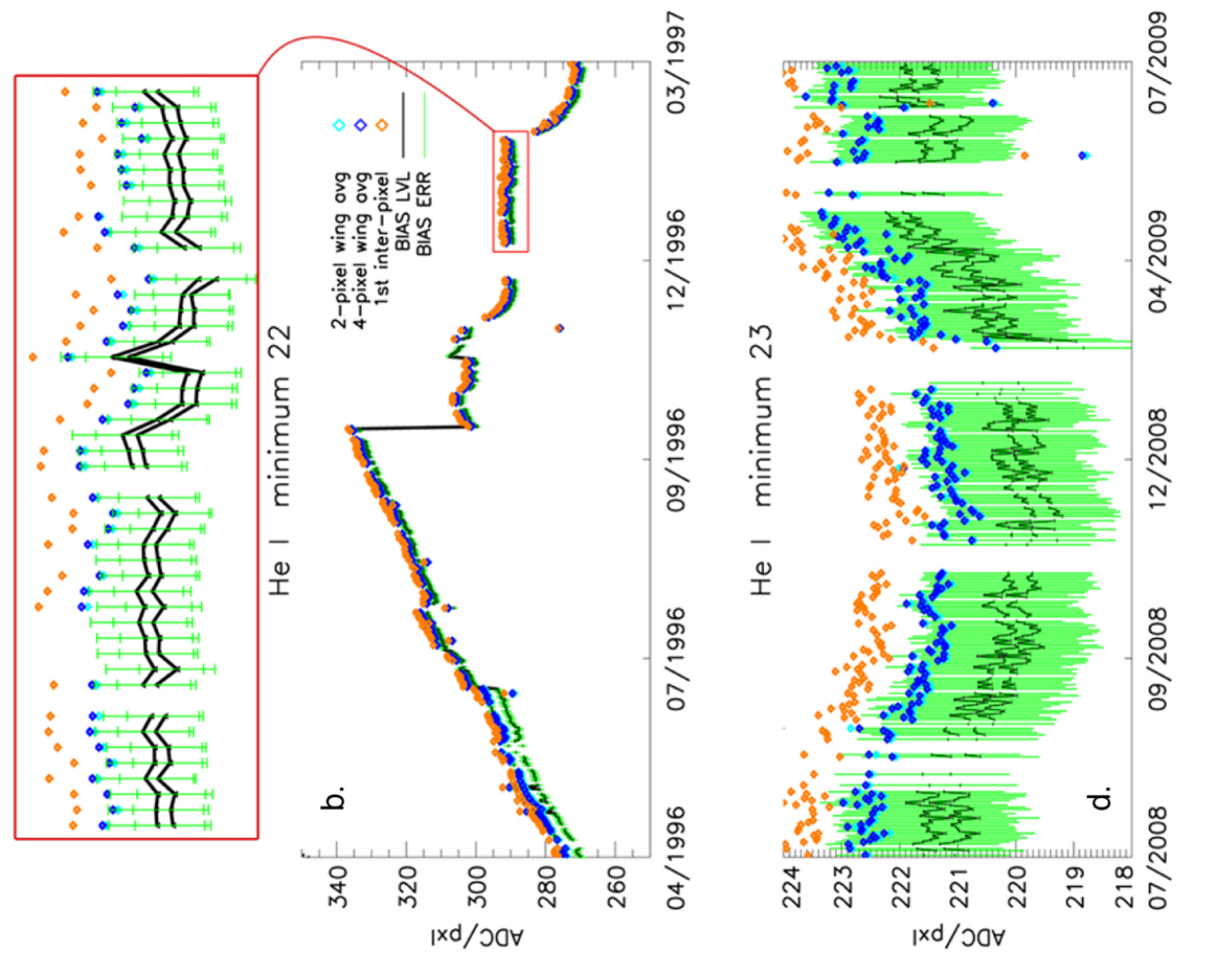

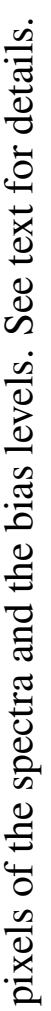
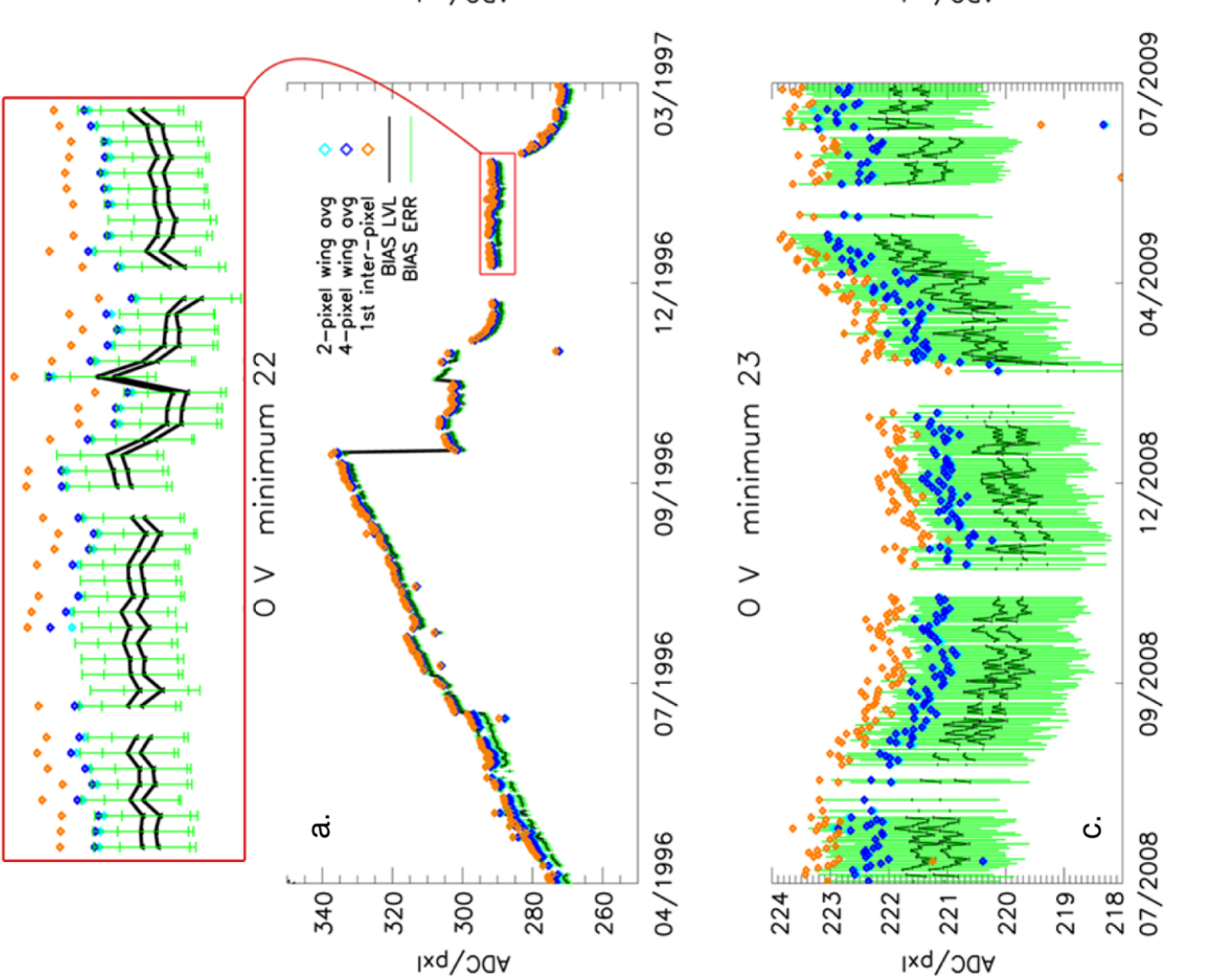

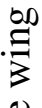
$\stackrel{8}{\Xi}$

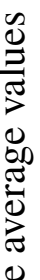

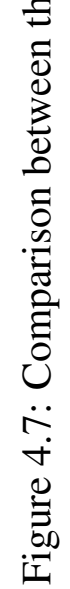



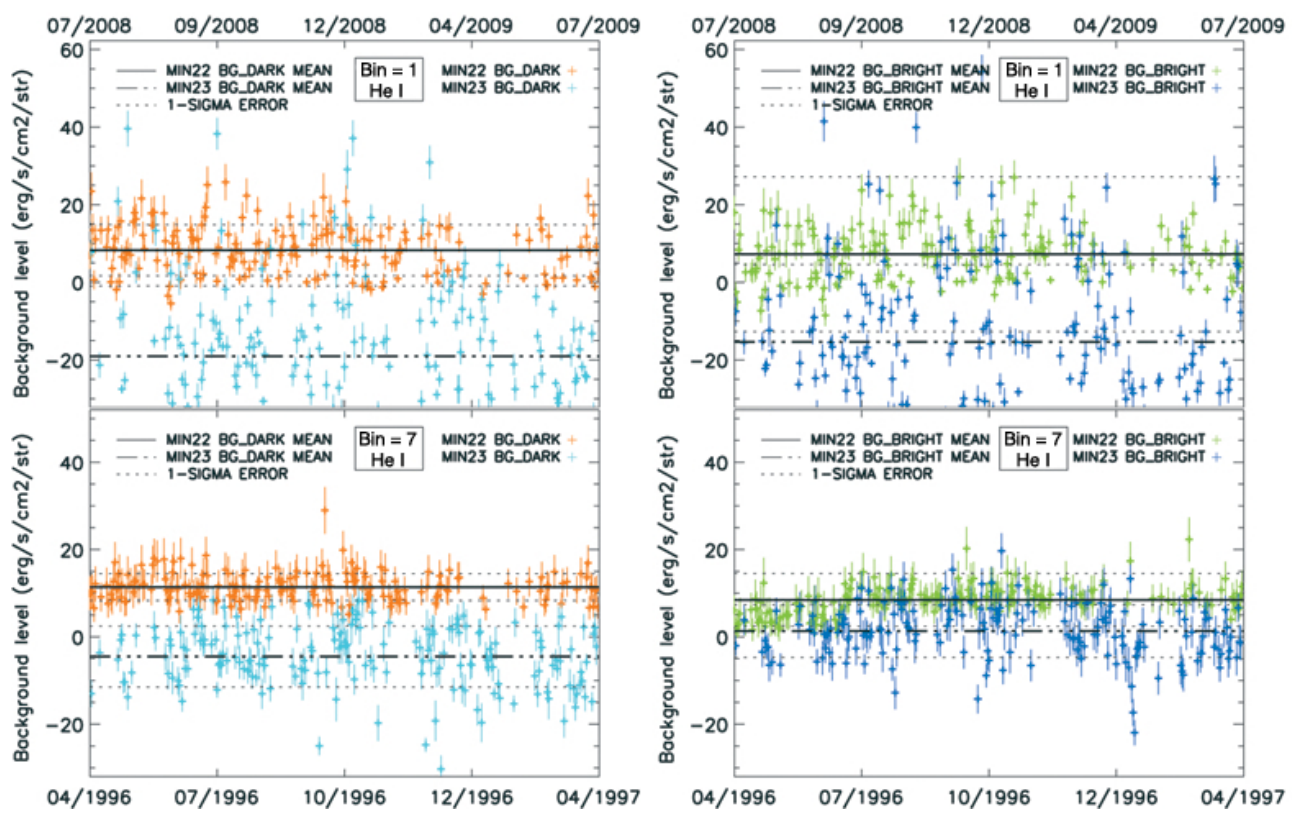

Figure 4.8: Fit backgrounds of the 5\% darkest (left) and 5\% brightest (right) spectra of the He I lines for the binsize values of 1 (top) and 7 (bottom). Note that, the background levels can be negative due to bias removal (see text for details). The solid, dash-dot and the dotted lines in all panels correspond to the average background over minimum 22 and 23 and their $1 \sigma$ standard deviation, respectively. The Orange and cyan plus signs, correspond to the background values of the bright spectra during the solar minima 22 and 23 , respectively. The background values of the dark spectra over solar minima 22 and 23 are shown by green and blue plus sign, respectively.

levels are positive for the spectra taken over minimum 22, while they are significantly less during minimum 23. The negative values of the background levels in minimum 23 are due to the debiasing of the data. Since the wing value are in the bias levels (see Fig. 4.7), the debiasing results in the negative values on the wing regions of the spectra. Consequently, when fitting the spectra, the background levels are usually estimated to be negative. Note that the background values are determined regardless of the total radiance of the spectra, since the two groups of dark and bright spectra have similar background levels.

Comparing the two binsize values in Fig. 4.8, it is evident that the binning of the data increases the background level during minimum 23. The resulting pixels (after binning) have higher signal-to-noise ratio and, therefore, debiasing the binned data results in less negative values and consequently, higher average background level. The results from the same analysis carried out for the $\mathrm{O} v$ spectra were similar, except that the average background levels of the spectra over minimum 23 and for binsize 1 was approximately twice larger than that of the He I spectra.

Finally, a look to the very weak spectra (from dark regions) taken during minimum 23, reveals that, in most cases, the signal is too low and the spectra do not resemble a Gaussian any more. Fig. 4.9 exhibits three examples of such spectra in the $\mathrm{O} v$ line, taken on 2008 Aug 29. These low signal spectra are dominant in the very dark regions of the intensity images in both spectral lines. The noise level in such spectra can be as high as, and even 

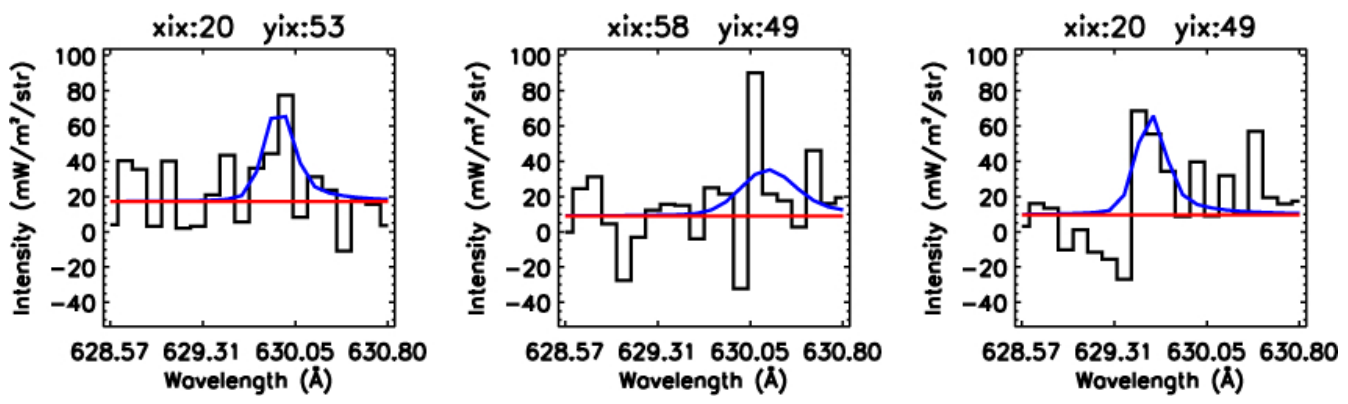

Figure 4.9: Examples of spectra in very dark regions of a given $\mathrm{O} v$ intensity image, taken on 2008 Aug 29. The blue and the red lines are the Gaussian fits and the background levels, respectively. The parameters xix and yix at the top of each panel, indicate the spatial coordinate of the spectrum on the intensity image, corresponding to the pixels in the $x$ and $y$ directions, respectively.

larger than, the amplitude of the line.

A simple simulation was run to examine if and to what extend, these high levels of noise can alter the contrast ratios. To do so, two average spectra from the 5\% darkest and 5\% brightest spectra of a given $\mathrm{O} v$ image, taken on $2009 \mathrm{Feb} 22$ were produced and then fitted with broadened-Gaussian functions (top panels of Fig. 4.10). Taking these as reference spectra, a random noise ranging within plus and minus of the amplitude of the fit to the "dark reference spectrum" were applied to both reference spectra in 1000 runs. It was assumed that both dark and bright spectra suffer from the noise level, which is in the order of magnitude of the dark spectrum peak.

This way, two sets of 1000 dark and bright spectra were produced. The spectra were then fitted following the procedure explained in Sec. 3.3.6 and their intensities were extracted $\left(I_{i}\right)$ and normalized to the intensity of the reference spectra $\left(I_{0}\right)$. Finally, the histograms of the normalized intensities were produced and fitted by Gaussian functions (bottom panels of Fig. 4.10). The histogram of the dark spectra has an average $I_{i} / I_{0}$ ratio of 0.84 , implying that the intensities of the weaker spectra with high level of noise, tend to be underestimated by about $16 \%$. The same level of noise does not remarkably change the average intensities of the bright spectra, since the average $I_{i} / I_{0}$ ratio is 0.99 . Assuming the ratio between the intensities of the bright and dark reference spectra to be the $5 \% \mathrm{CR}$, the resulting underestimated intensities (by $16 \%$ and $1 \%$ for the simulated dark and bright spectra, respectively), leads to the overestimation of the $5 \% \mathrm{CR}$ by $17 \%$. This difference is very close to the difference between the ratio of the 5\% CRs for binsize 1 and 2, seen in Fig. 4.6. Relying on these results, it can be deduced that binning suppresses the effect of high noise level in the data taken over minimum 23 to a high extent.

\subsubsection{Effect on CRs of spectral broadening after SOHO recovery}

In Sec. 3.3.6 it was explained that the NIS spectra after the loss of CDS became asymmetric and broadened. This modification also results in a reduction of the spectral resolution of the instrument. This effect, although unlikely to be important to our analysis, was nevertheless checked to see if it significantly affects the CRs. For this purpose, the data from cycle minimum 22 were artificially broadened, new CRs were calculated and were 

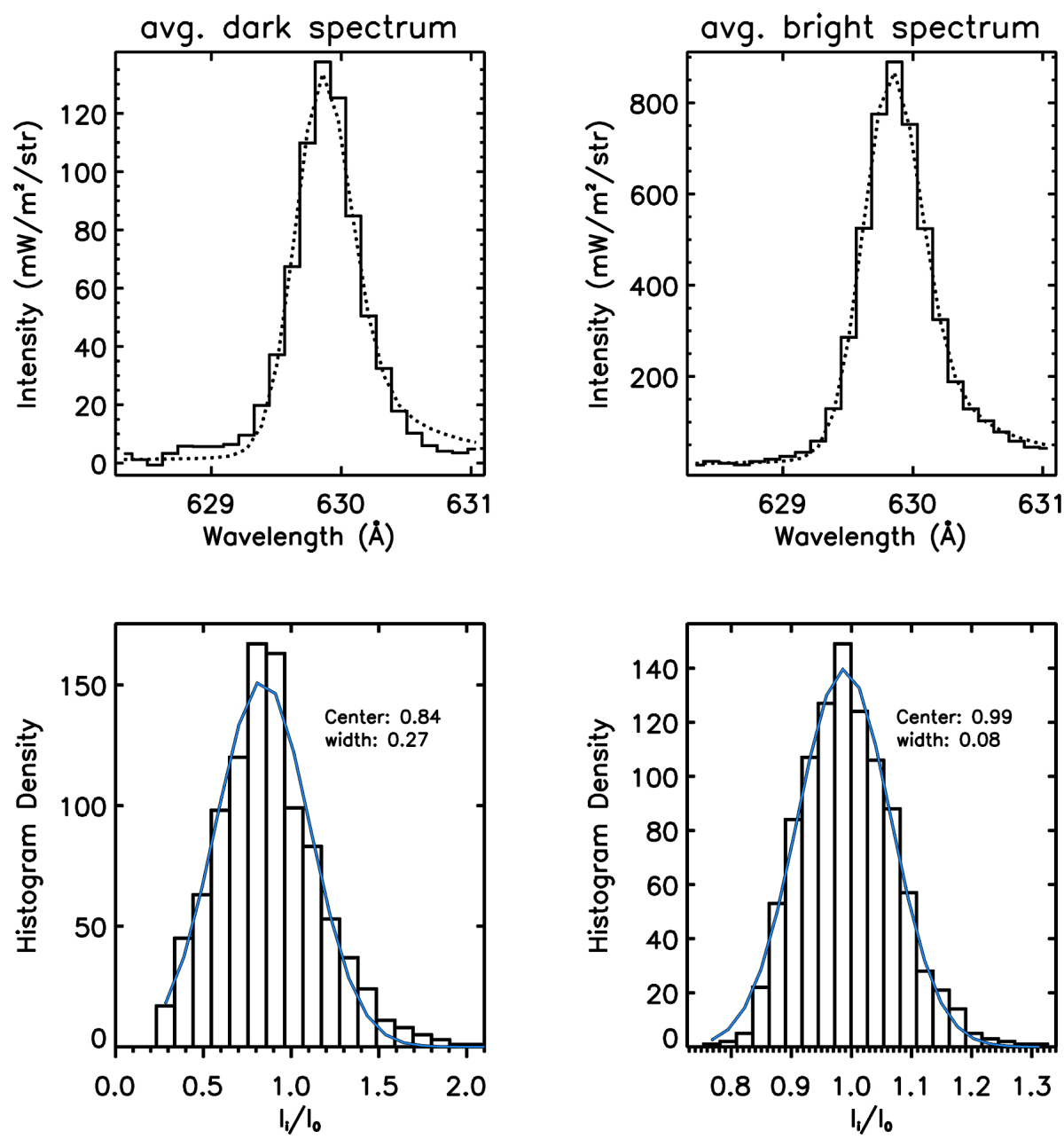

Figure 4.10: Top: Average 5\% dark (left) and 5\% bright (right) spectra, produced from the O v intensity image taken on $2009 \mathrm{Feb} 22$. The dotted lines are the broadened-Gaussian fits. Bottom: The histograms of the normalized intensities $\left(I_{i} / I_{0}\right)$ of the simulated dark (left) and bright (right) spectra. The solid blue lines are the Gaussian fits to the distributions; the peak position and width of the fitted Gaussians are also indicated

compared with the original CRs. The procedure is illustrated in panels $a$ ) to $d$ ) of Fig. 4.11 and explained in this section.

First, a random spectrum, taken during minimum 22, was chosen to be artificially broadened (panel $a$ ). The wavelength dimension was extended over 24 pixels (instead of 18 for pre-loss data), corresponding to the data taken after CDS recovery. Having the already extracted parameters of the Gaussian fit of the chosen spectrum $\left(\gamma_{0}, A_{0}\right.$ and $\left.\sigma\right)$, as well as the standard values of asymmetry $\alpha$ (see Sec. 3.3.6), Equations 3.3, 3.4 and 3.5 were used to produce the corresponding broadened and asymmetric spectrum (panel $b$ ).

In Sec. 4 of CDS Software Note $53^{1}$, it is discussed that the full-width-half-maximum (FWHM) of the broadened spectra (as a measure of spectral resolution) after the recovery of the instrument is $2.604 \sigma$. This is larger than the pre-loss FWHM of the spectra by a factor of 1.105 . Therefore, the artificially broadened spectrum was convolved by a

${ }^{1}$ http://solar.bnsc.rl.ac.uk/swnotes/cds_swnote_53.pdf 

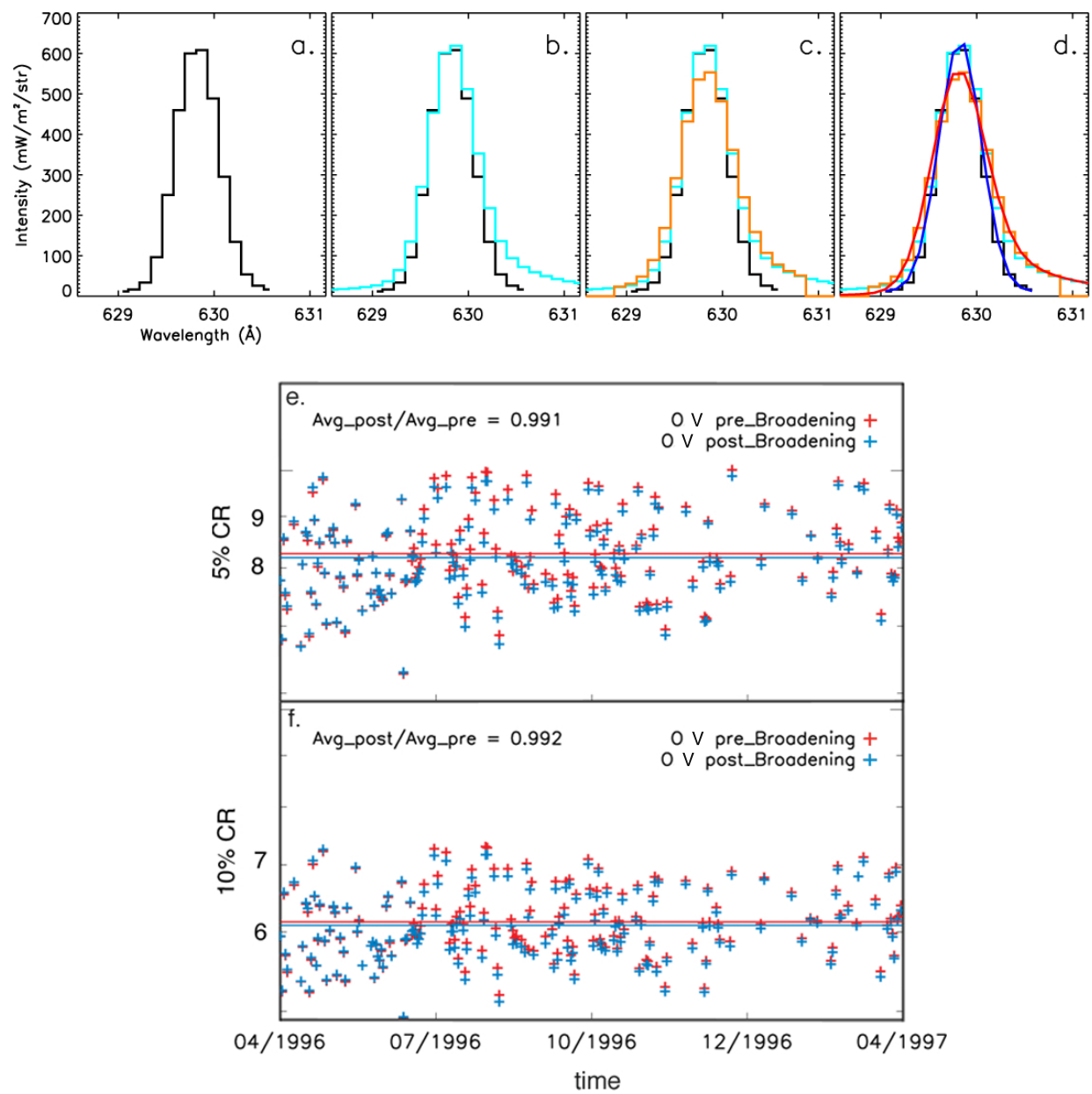

Figure 4.11: Effect on contrast ratios of spectra being broadened after SOHO loss. a) Random spectrum; b) Artificially broadened spectrum (cyan); c) Broadened spectrum convolved with a Gaussian (orange); d) Gaussian (dark blue) and broadened-Gaussian (red) fits to the original (black) and artificially produced (orange) spectra, respectively; Comparison between the 5\% (e) and 10\% (f) CRs of the original and artificial spectra. Pre-broadened spectra, red crosses; post-broadened, blue crosses

Gaussian with the width value of 1.105, to reduce the spectral resolution of the spectrum accordingly (panel $c$ ). Finally, the new spectrum was fitted with a broadened-Gaussian (panel $d$ ) and the intensity of the broadened spectrum was calculated. This procedure was done for all $\mathrm{O} v$ data taken in minimum 22 , the $5 \%$ and $10 \% \mathrm{CRs}$ were determined and compared to the original CRs. The results are shown in panel $e$ ) of Fig. 4.11. The difference between the CRs of the original and the artificial data is less than $1 \%$ and can not explain the difference between the CRs of the two minima.

\subsubsection{Co-spatiality between $\mathrm{O} v$ and $\mathrm{He} \mathrm{I}$}

Since we discuss the behaviour of the CRs obtained for two different lines, it is of relevance to check the level of co-spatiality of the brightest and darkest areas visible in these two lines. Fig. 4.12 exhibits four intensity images in $\mathrm{O} v$ after being binned over $2 \times 2$ pixels 

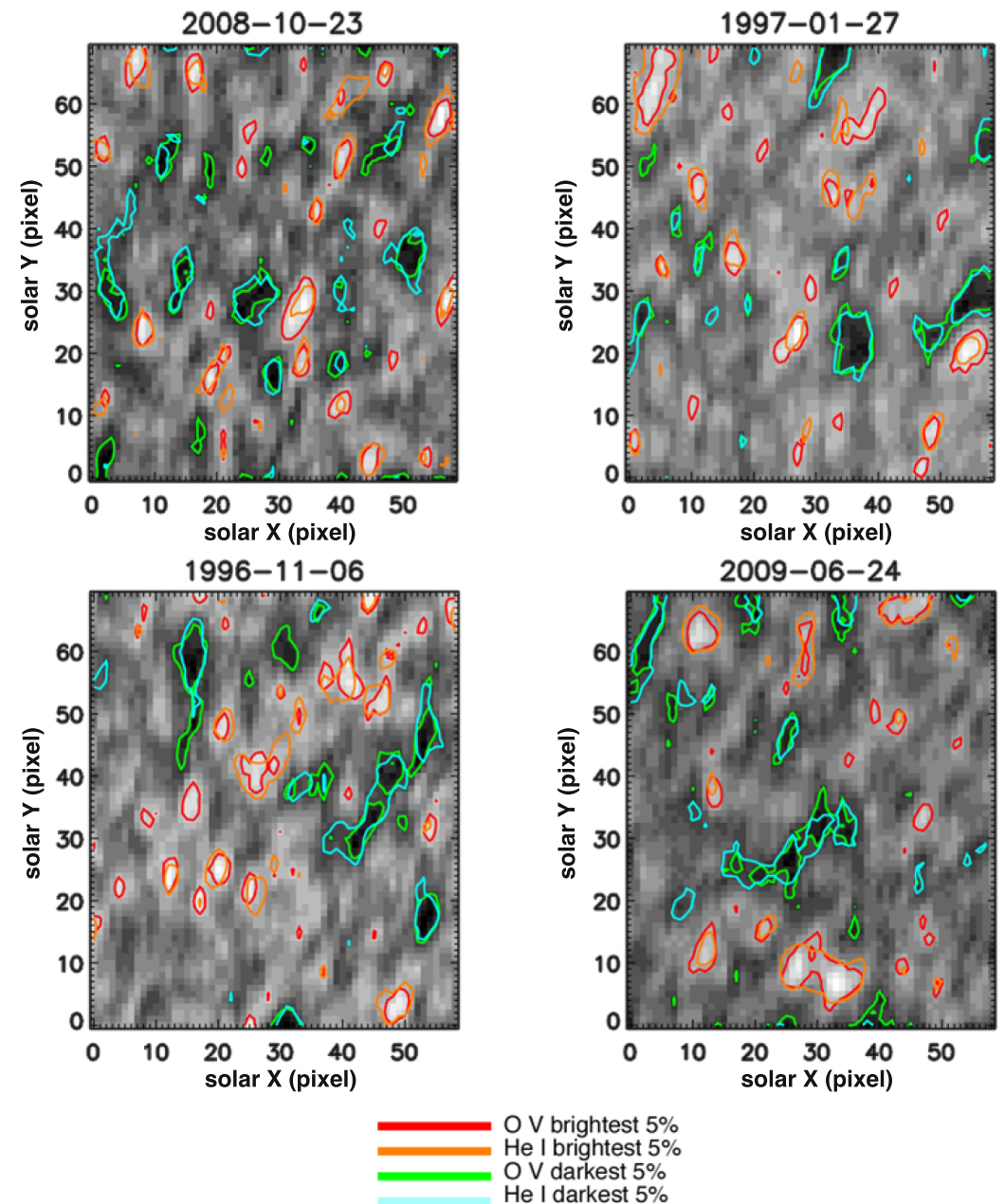

Figure 4.12: Overlap between the 5\% brightest (and 5\% darkest) regions of the $\mathrm{O} v$ and He I intensity images. The colour coding of the different types of contours is given below the figure.

(binsize 2). The contours of the 5\% brightest and 5\% darkest regions of the background $\mathrm{O} v$ image are shown on each image. In addition, the respective contours of the He I image from the same observation are also overplotted (He I image not plotted). It is clear that, as expected, the darkest and brightest pixels cluster on the same areas in both lines. However, it is also evident that there are differences in the shape and extension of these areas.

For each intensity image, the co-spatiality between the two spectral lines in the brightest and darkest regions of the intensity images, was defined as the percentage of the number of pixels within the 5\% brightest and 5\% darkest contours with the same spatial coordinates on the intensity images of both spectral lines, to the total number of pixels within the $5 \%$ contours, respectively. Note that, since the intensity images always have the same dimensions, the total number of pixels inside the 5\% contours is a constant number, regardless of the choice of the spectral line. Similarly, the co-spatiality between the $10 \%$ brightest and 10\% darkest regions was calculated. Fig. 4.13 depicts the co-spatiality between the $\mathrm{O} v$ and $\mathrm{He}_{\mathrm{I}}$ spectral lines over the last two solar minima and for two binsize values of 1 and 3 . The percentage of co-spatiality was calculated for $5 \%$ and $10 \%$ brightest 
and darkest regions separately.

At a first glance, the co-spatiality between the brightest regions of the intensity images of the two spectral lines, corresponding to the network regions, is almost identical in both minima (see panels $a$ ), $b$ ), $d$ ) and $f$ ) in Fig. 4.13). Obviously, the overlap increases as the size of the contours become larger (from 5\% to 10\%) and as the images are being binned. In the darker regions, the co-spatiality between the unbinned data drops significantly in minimum 23 (panels $c$ ) and $d$ )). As discussed in the previous sections, the signal level in the dark regions during minimum 23 is so low that the weak spectra, likely, produce random patterns which are not overlapping each other. Panels $g$ ) and $h$ ) in Fig. 4.13 show that, as soon as the data are binned, the spatial overlap between the dark regions of the spectral lines become very similar in both minima. In addition, by comparing the panel $e$ ) with the panel $g$ ) (as well as $f$ ) with $h$ )), it turns out that the overlap between the dark regions and bright regions are identical after binning.

\subsection{Intensity distribution of the quiet Sun radiation and lognormal fitting}

Various studies have shown that the intensity distribution of the quiet Sun in the chromosphere and transition region has a skewed shape, with a pronounced peak at relatively low intensities and an extended tail at higher intensities. This skewed distribution has been modelled mainly by either a sum of two Gaussian functions (Reeves 1976, Gallagher et al. 1998) or a single Lognormal function (Griffiths et al. (1999), Pauluhn et al. 2000, Fontenla et al. 2007), each representing a different hypothesis about the source of the radiation in quiet Sun regions. These two models imply different heating scenarios. A two-component function assumes two intrinsically different heating mechanisms for radiation originating in the solar network and internetwork, while a single-component model assumes similar heating mechanisms for both network and internetwork (Pauluhn et al. 2000). The common agreement today is that the quiet Sun radiation follows in general a lognormal distribution function. Thus, Pauluhn et al. (2000) showed that despite its fewer free parameters, a lognormal reproduced the observed distributions significantly better than the sum of two Gaussians. As mentioned earlier, in order to study the variation of the radiance distribution over time, the individual distributions were fitted with a lognormal function (see Sec. 1.5.1), extract the best fit parameters and investigate their long-term variability.

Fig. 4.14 illustrates two intensity images in the $\mathrm{O} v$ spectral line together with their corresponding histograms and two MDI magnetograms of a slightly larger solar area, encompassing the area scanned with CDS. The upper row (panels a), b) and c)) corresponds to a date (1996 June 29) on which part of an active region passed through the field of view, while the bottom row (panels d), e) and f)) depicts quiet Sun conditions (observations taken on 1996 July 14). Panels c) and f) show two histograms: the actual intensity histogram shown by the solid black line and the normalized histogram of the natural logarithm of the intensity $(\log (I))$ represented by the solid red line. The black dashed lines are Gaussian fits to the normalized histogram of the logarithm of the intensity distribution. A comparison between panels c) and f) reveals how significantly the distributions can deviate from a lognormal distribution when a particularly bright structure, such as a part of an AR, appears in the field of view. $\chi^{2}$ values, as will be explained in Sec. 4.4, were used to exclude the 

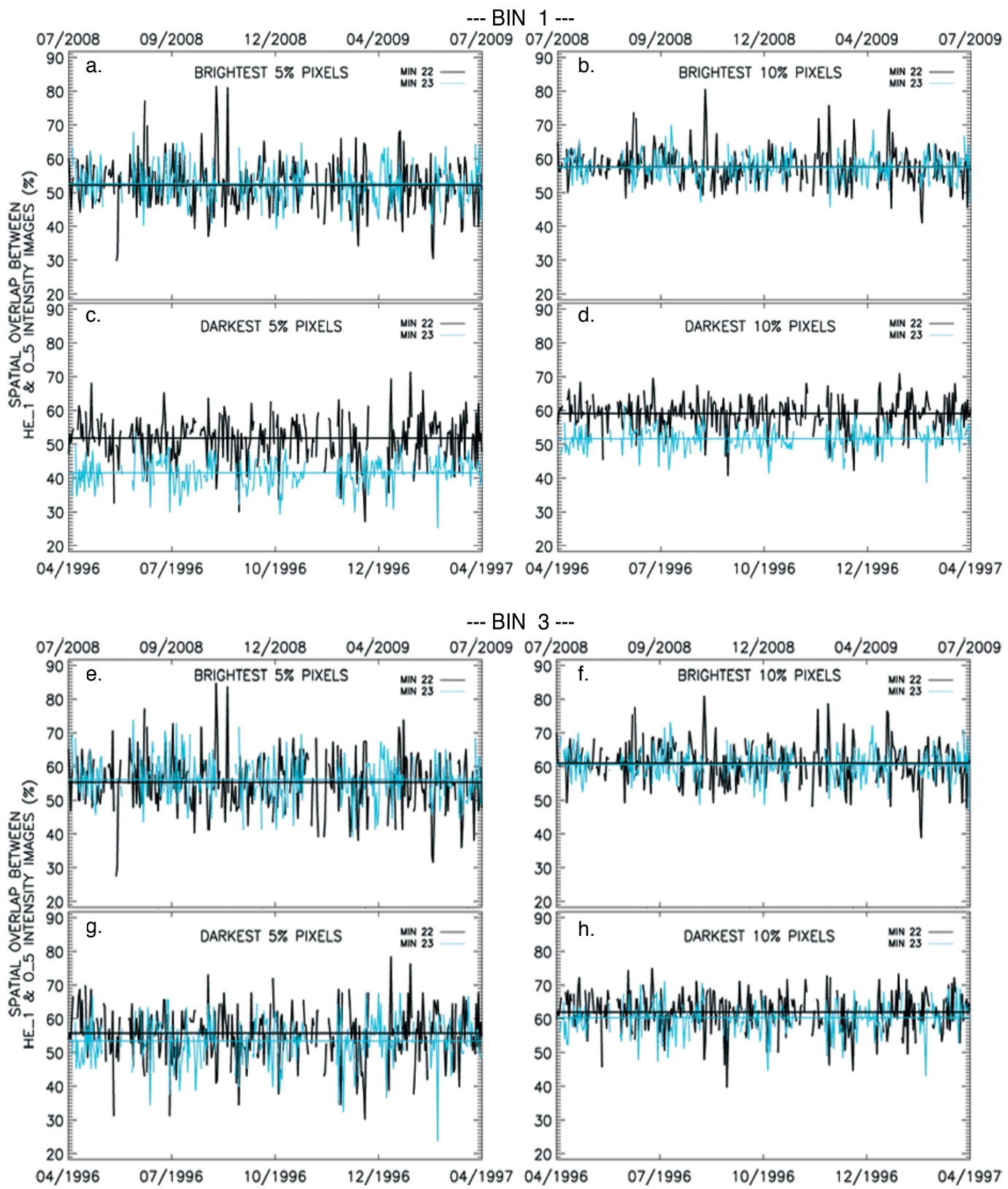

Figure 4.13: Variation of the co-spatiality between the $\mathrm{O} v$ and $\mathrm{He}$ I intensity images during the solar minima 22 and 23. The amounts of spatial overlap were calculated and plotted for 5\% (left panel) and 10\% (right panel) brightest and darkest regions, separately, and for two different binsize values of 1 (top panels) and 3 (bottom panels). The horizontal black and blue lines correspond to the mean values during solar minima 22 and 23 , respectively. 

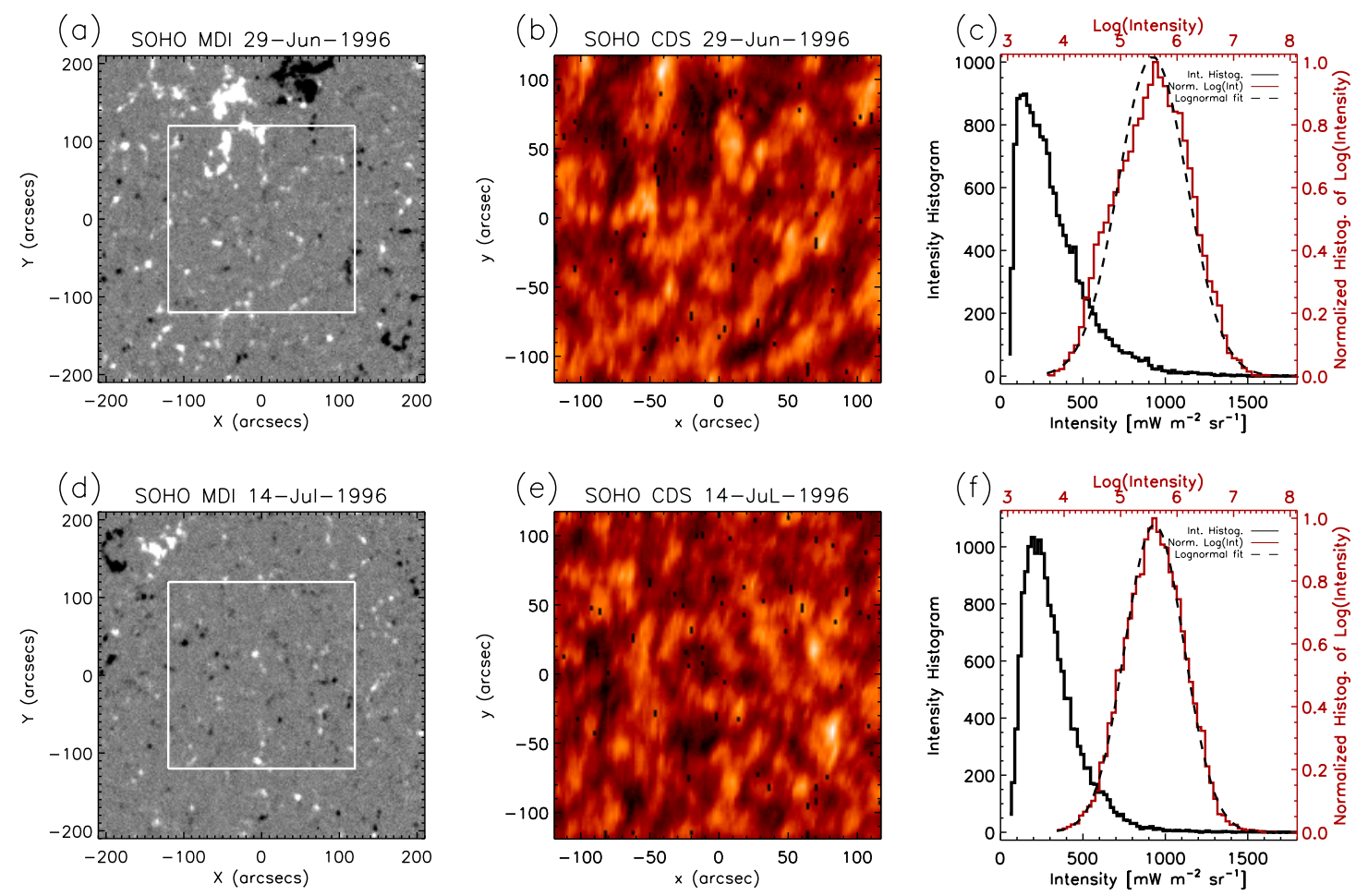

Figure 4.14: From left to right: MDI magnetograms, $\mathrm{O} v$ integrated intensity scans and intensity distribution functions obtained from the intensity images. The solid black lines in panels $\mathrm{c}$ and $\mathrm{f}$ show the intensity histograms on a linear intensity scale (corresponding to the axes on the bottom and left side of panels $\mathrm{c}$ and $\mathrm{f}$ ). The solid red lines are the normalized histograms of the logarithm of the intensity (corresponding to the axes on top and right side of panels $\mathrm{c}$ and $\mathrm{f}$ ). The dashed lines are lognormal fits to the normalized histograms. The surface area visible in CDS images is marked by the white rectangles in the MDI magnetograms.

non-quiet Sun data from the analyses, as an alternative approach to visual inspection.

The scale $\mu^{*}$ and the shape $\sigma^{*}$ parameters of the individual intensity distributions were calculated according to the $\mu$ and $\sigma$ values of the normal fits, as formulated before (see Sec. 1.5.1). Note that the scale parameter is sensitive to the instrumental calibration. Referring to the mathematical definitions of the shape and scale parameters (Eq. 1.4), multiplying an intensity image by a given number (as a correction factor) shifts the position of the peak of the histogram and, consequently, changes the scale parameter, while the shape parameter remains unchanged. This can be seen in Fig. 4.15, which exhibits the variations in the scale and the shape parameters of the intensity distributions of the He I spectral line. The scale parameter shows a similar trend to that of the average intensities of He I shown in Fig. 4.3, while the shape parameter does not depict any systematic trend.

Various statistical parameters were defined using the $\mu$ and the $\sigma$ values (see Sec. 1.5.1). The parameters that are dependent on $\mu$ (i.e., scale parameter $\mu^{*}$, mean $\bar{I}$ and mode $M O D$, defined by Eqs. 1.4, 1.7 and 1.8, respectively) were dismissed for further analyses, for the same reason as the average intensities were not analysed. These parameters may eventually be used to improve the instrument calibration, if one assume that, for instance, the average 


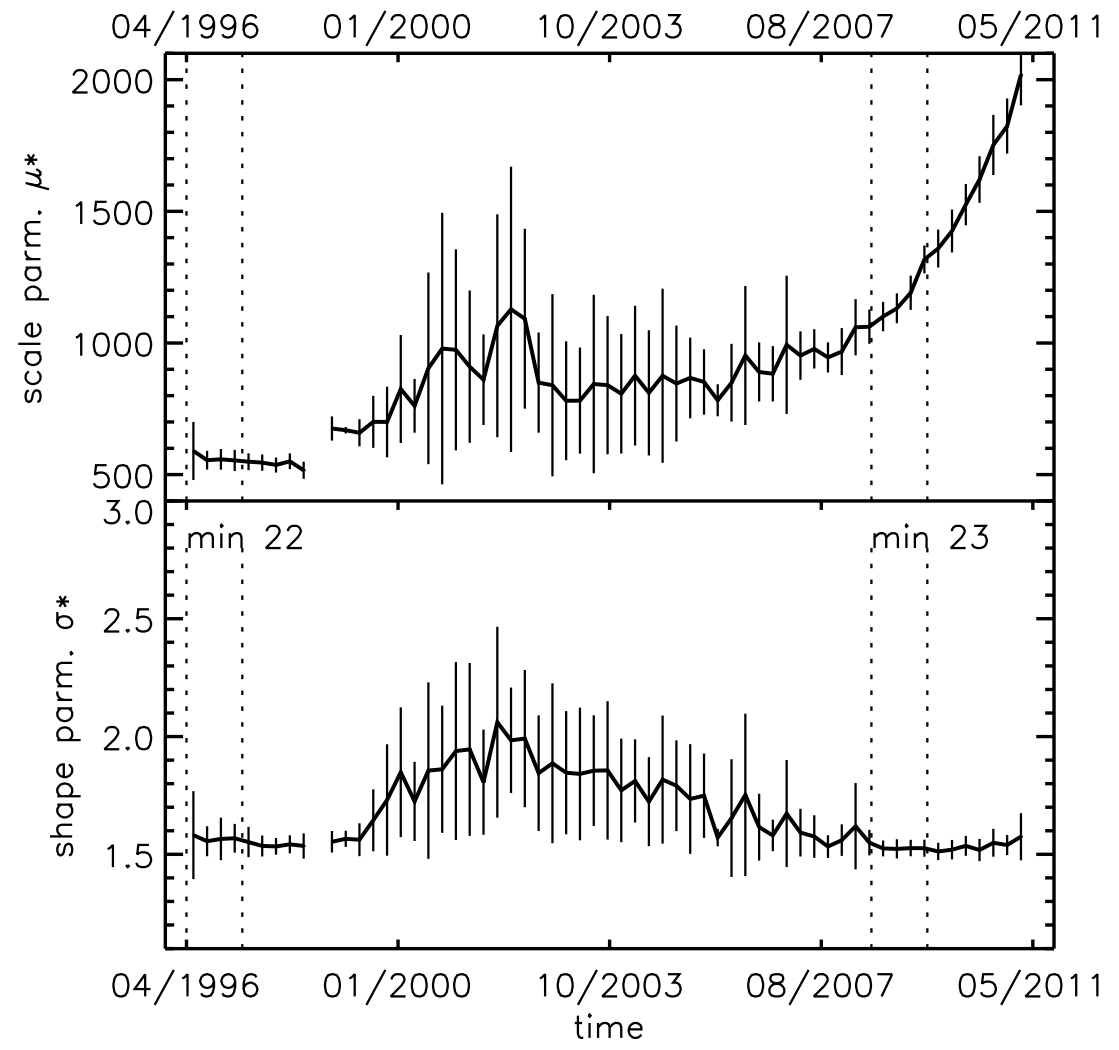

Figure 4.15: Long-term variations in the scale (upper panel) and shape (lower panel) parameters of the lognormal fit to the intensity distribution of the He i spectral line. The solid lines and vertical error bars correspond to 90-day average values and standard deviations, respectively. The vertical dashed-lines indicate the two 1-year time spans covering the solar minima 22 and 23.

quiet Sun radiance is cycle-invariant, as was done by Del Zanna et al. (2010). Since the purpose of this thesis is actually to investigate whether there are cycle-related variations in the radiance distribution of the quiet Sun, it was decided to study the parameters that only depend on $\sigma$. Four such statistical parameters were chosen for this study: the shape parameter $\sigma^{*}$, the skewness parameter $\gamma$, the coefficient of variation $(C V)$ (defined by Eqs. 1.4, 1.5 and 1.6, respectively) and the $\chi^{2}$ value of the fits. In general, the distribution is more asymmetric and dispersed for larger $\sigma$, while a smaller $\sigma$ implies that the distribution is more symmetric.

Fig. 4.16 displays the ratio of the average lognormal fitting parameters of minimum 23 to those of minimum 22, for different binsizes. Plotted are such ratios of $\sigma^{*}$ (panel $a$ ), $\gamma$ (panel $b$ ) and $C V$ (panel $c$ ) averaged over the periods of time defined as minima. Excluding the $\chi^{2}$ plot which gives a measure of the goodness of the fits, all other plots depict lower than unity ratios for all binsizes greater than 1 , specially for $\mathrm{Ov}$. This suggests that the intensities follows a less asymmetric distribution during solar minimum 23, i.e., the intensity distributions are closer to a normal distribution. This effect is less pronounced in $\mathrm{He} \mathrm{I}$, with closer-to-unity ratios compared to the $\mathrm{O} v$ spectral line results. This implies 

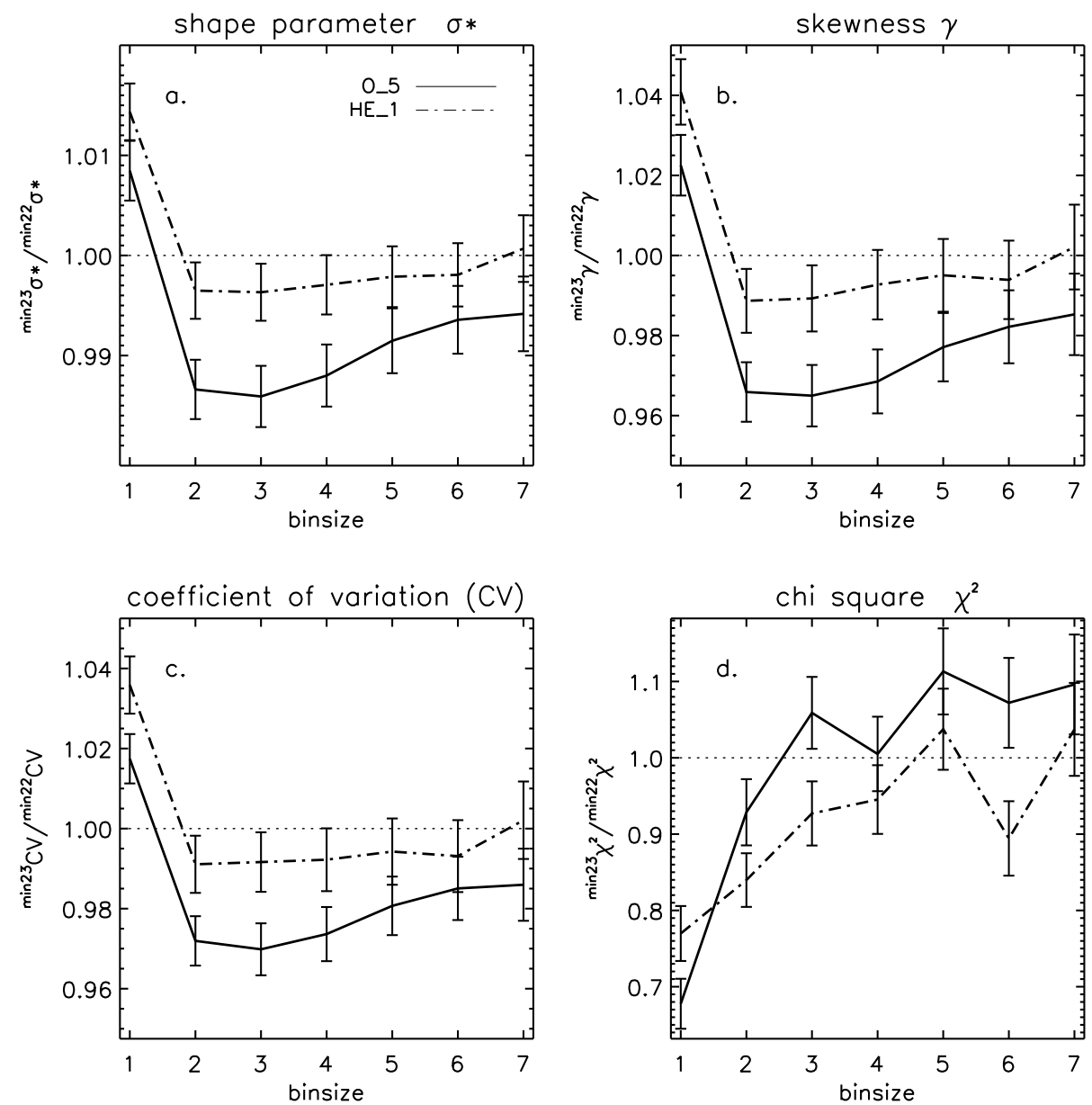

Figure 4.16: The cycle 23 activity minimum to cycle 22 activity minimum ratios of various statistical parameters of the lognormal fits: the shape parameter $\sigma^{*}$ (panel $a$ ), the skewness $\gamma$ (panel $b$ ), the coefficient of variation $\mathrm{CV}$ (panel $c$ ) and the $\chi^{2}$ goodness of the fits (panel $d$ ). The solid and dash-dotted lines represent the ratios in $\mathrm{O} v$ and $\mathrm{He}$ I spectral lines, respectively. The error bars represent the $1 \sigma$ error in the ratios.

that the mean values at the two ends of the intensity distributions are closer in magnitude, so this result is in agreement with the $5 \%$ and $10 \%$ CRs being smaller at minimum 23 , as shown in Fig. 4.6. Here again the exception is the binsize 1, which shows a similar behaviour to that of the CRs. The $\chi^{2}$ ratio helps to determine which amount of binning is most appropriate for comparing the two minima. The best consistency between the data for two minima, in terms of signal to noise ratio, can be achieved for $3 \times 3$ and $4 \times 4$ spatial binning for both spectral lines.

\subsection{Data exclusion based on $\chi^{2}$ of the lognormal fits}

As already shown in Fig. 4.14, the intensity distributions of the images with any trace of activity exceeding that of the typical quiet Sun deviate from a lognormal. The $\chi^{2}$ values of the lognormal fits, as mentioned before, can therefore also be utilized as a potential reference for excluding scans that do not cover purely quiet Sun. Fig. 4.17 shows the 

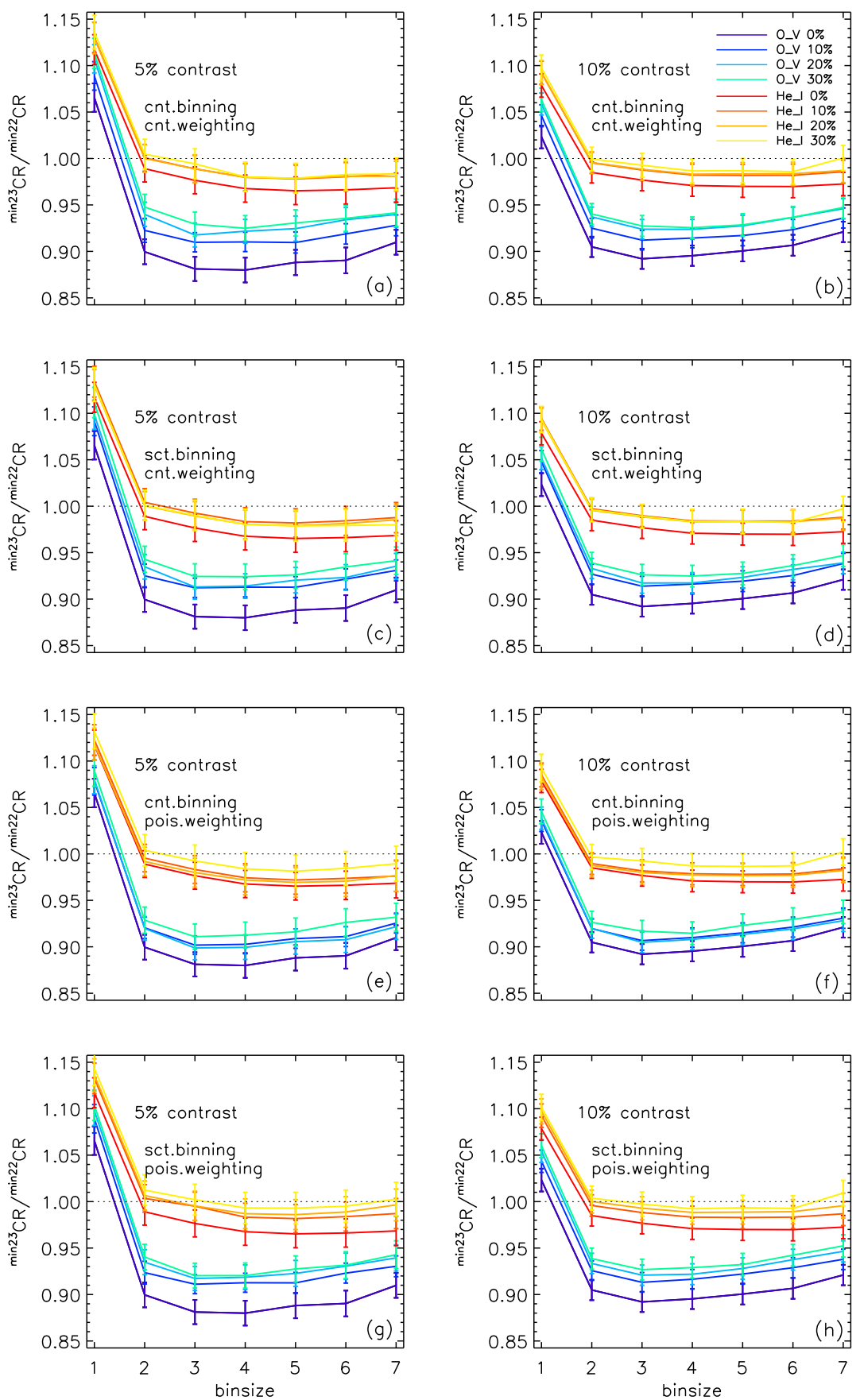

Figure 4.17: Minimum-to-minimum ratio of the 5\% and 10\% CRs. Each panel, consists of the ratios of the $\mathrm{Ov}$ and $\mathrm{He}$ I spectral lines and for different amounts of data exclusion (from $0 \%$ to $30 \%$ ), based on the $\chi^{2}$ values of a lognormal fit to radiance distributions. The effect of different methods of determining the histogram binsize and weighting the histograms for lognormal fitting has been emphasized (see text for details). The abbreviations cnt, sct and pois stand for constant, Scott's rule and Poisson, respectively. The meaning of the different coloured lines are indicated in panel $b$. 
minimum-to-minimum ratios of the $5 \%$ and $10 \% \mathrm{CRs}$, similar to the one which is already shown in Fig. 4.6, but this time instead of excluding the data by visual inspection, the selection was done according to the $\chi^{2}$ of their lognormal fits. Here again three different versions were introduced corresponding to the number of data points which were excluded. $0,10,20$ and $30 \%$ in the images label implies that this fraction of the intensity images with highest $\chi^{2}$ values, was removed before the CRs were computed. In order to check if the ratios are sensitive to the histogram bin width and/or to the weighting of the bins for lognormal fitting, two different methods for defining the histogram bin width and two methods to weight the histogram bins were tested. The bin widths were either given a fixed value equal to $20 \mathrm{~mW} / \mathrm{m}^{2} / \mathrm{sr}$ (labelled cnt.binning in Fig. 4.17) for the entire data set and for both spectral lines or, alternatively, determined according to Scott's normal reference rule (Scott 1979) for each individual histogram (labelled sct.binning). Scott's normal reference rule estimates the optimal bin width for a histogram by assuming the underlying distribution to be a Gaussian and by minimising the integrated mean square error (IMSE) of the histogram (Scott 1979). For lognormal fitting, the bins were either given a constant weight (labelled cnt.weighting) or a statistical (Poisson) weight (labelled pois.weighting). The four different combinations of these methods for the two CRs are illustrated in Fig. 4.17. In most cases, there is a remarkable gap between the $0 \%$ line, referring to the ratio of CRs obtained without removing any data (i.e., keeping also the brightest scans, most strongly affected by active regions) and the other lines. The fact that the 10\%, 20\% and 30\% lines almost overlap each other, implies that the ratio between the CRs approaches a saturation level when going to larger exclusion percentages. This was less evident for the $\mathrm{O} v$ line in Fig. 4.6, where the exclusion was done by visual inspection. Here again, regardless of which method for determining the bin widths and bin weights is used, the He I line does not show any significant difference between the two minima. The $\mathrm{O} v \mathrm{CRs}$, on the other hand, exhibit a drop of approximately $8 \%$ during minimum 23 compare to that of minimum 22 , which is almost twice the change that was seen in the CRs when excluding the dataset containing brights area by visual inspection only.

\subsection{Network thickness}

The network boundaries, as discussed in Sec. 1.3.2, are the regions along the edges of the supergranular cells where the magnetic field is advected by the convective motions. From there it expands and extends to fill the upper solar atmosphere. Therefore, having a knowledge about the variation in the size of the network between the two minima, may provide indirect information of the magnetic field concentration along the network lanes. The thickness of the network boundaries in the solar atmosphere has been studied by Kundu (1985), Patsourakos et al. (1999) and Gontikakis et al. (2003). All studies take advantage of the autocorrelation function (ACF) of the images in estimating the size of the network structures.

The AFC of an image is basically the cross-correlation of the image with itself. To calculate the cross-correlation between two different images, the following steps need to be taken. First, one of the images, with dimensions of $(N, M)$, is taken as the reference. The second image, will then be centred at pixel $(i, j)$ (starting from $(0,0)$ to $(N-1, M-1)$ ) of the reference image, and the cross-correlation will be calculated and assigned to the 
pixel $(i, j)$. Eq. 4.1 shows how the cross-correlation factors $\left(r_{i j}\right)$ between a reference image $R$ and a mask image $M$ with a smaller dimension $\left(N_{i}, M_{j}\right)$ and pixel indices of $(i i, j j)$, is calculated.

$$
r[i][j]=\sum_{j j=-M_{j} / 2}^{j j<M_{j} / 2} \sum_{i i=-N_{i} / 2}^{i i<N_{i} / 2}\left(M\left[i i+N_{i} / 2\right]\left[j j+N_{j} / 2\right]-\bar{M}\right)(R[i+i i][j+j j]-\bar{R}) .
$$

This way, a 2D pattern of the cross-correlation factors with the same dimension of the reference image is produced ${ }^{1}$. Now, if the reference and the mask images are identical, the produced pattern consists of autocorrelation function and is referred to as the ACF of the image. However, using Eq. 4.1 to calculate the ACF of an image is very time-consuming. The alternative approach is to produce the ACF using the Fast Fourier Transform (FFT) of the image.

Starting with 1D data sets and assuming that $c(t)$ is the correlation function between two real functions $g(t)$ and $h(t)$, then

$$
c(t)=\int_{-\infty}^{\infty} g(\tau) h(\tau+t) d \tau
$$

where $\tau$ is the lag between the two functions. The Fourier transform of $c(t)$ is:

$$
C(f)=G(f) H(f)
$$

where $f$ is the frequency. Note that, since the initial functions are real, the imaginary parts in Eq. 4.3 are zero. If the functions $g(t)$ and $h(t)$ are the same, then the result will be the autocorrelation factor.

In case of a 2D data set (image), the data is first transformed into the frequency domain from the spatial domain using the FFT. It is then multiplied by its complex conjugate and the ACF will be produced by inverse transform of the product (see section 13.2 of Press et al. 1988). The ACF is then averaged over the azimuth angle, with the origin at the center of the ACF. The Half-Width-Half-Maximum (HWHM) of the central peak is the thickness of the network elements.

Before proceeding with the analysis of the data, this technique was applied to an artificially made image (in analogy to what was done by Patsourakos et al. 1999). Fig. 4.18 illustrates a sample image (panel $a$ ) and its ACF (panel $b$ ), as well as the angularly averaged ACFs in the linear and logarithmic scales (panels $c$ and $d$, respectively). The thickness of the bright strips and the sides of the squares in the sample image were set to be equal to 5 and 50 pixels, respectively. It was expected that the HWHM of the central peak of the angularly averaged ACF to be close to the feature size (network thickness). The angular averaged of the ACF also contains a secondary peak that corresponds to the mean distance between the bright features (network cell's dimension) (Gontikakis et al. 2003). Both parameters, as seen in the figure, are in good agreement with the characteristics of the sample image. Note that, averaging the ACF over the azimuthal angle results in a maximal

1 see lecture notes by Paul Bourke: http://paulbourke.net/miscellaneous/correlate/\#auto 

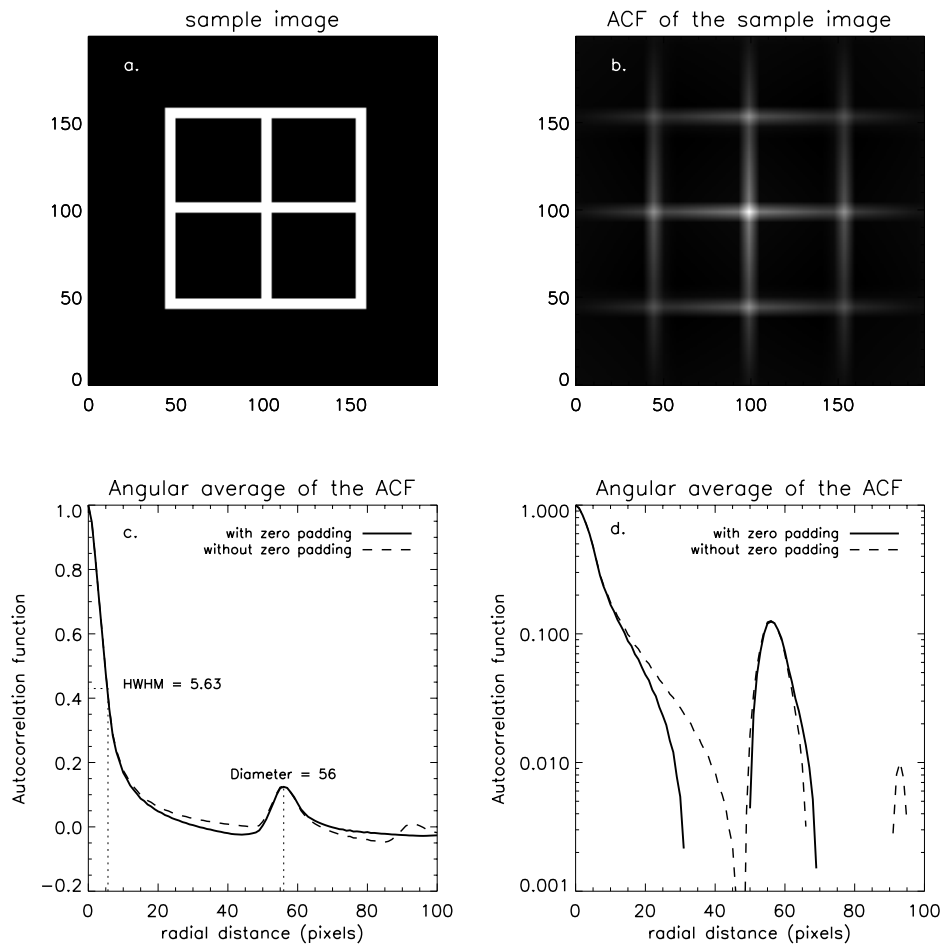

Figure 4.18: a) Simulation of the network structure. b) autocorrelation function (ACF) of the simulated image. Average of the ACF over azimuth angle in linear (panel $c$ ) and logarithmic (panel $d$ ) scales. The Thick and thin solid black lines correspond to the angular average of the ACF with and without zero padding, respectively.

feature size (HWHM), since this method is optimal for structures with circular patterns. For other structures such as the network elements, it results in an estimation of the scale length of the features (Patsourakos et al. 1999, Gontikakis et al. 2003). The network cell's dimension was not analysed in this study.

When using the FFT, one should also consider the edge effect, since the data have finite dimensions. Therefore, the image was padded with zeros in order to prevent the overlap of the convolution results of one edge with the opposite edge of the images. The effect of the zero-padding is shown in panels $c$ ) and $d$ ) of Fig. 4.18. For analysing the intensity images, all images were padded by zeros to overcome the edge effect.

Fig. 4.19 exhibits the results of applying the above mentioned method to two radiance images in the O v line, taken on $1996 \mathrm{Jul} 22$ (top panels) and 1996 Aug 16 (bottom panels). Prior of producing the ACF, the discontinuities at the edges of the images were smoothed using a cosine-bell function, such that a narrow strip of every image close to its borders (covering almost $10 \%$ of the image) were smoothed in order to deal with the limited size of the images (Brault and White 1971, Gontikakis et al. 2003). These smoothed area are visible in panels $a$ ) and $d$ ) of Fig. 4.19.

The radiance image in panel $a$ ), corresponds to a quiet Sun condition. The calculated network thickness (HWHM of the curve in panel $c$ )) is equal to $10.51^{\prime \prime}$. In panel $d$ ), an active region has appeared in the image. The bright feature is not a network structure and the HWHM of the ACF curve is much larger than a quiet Network element (with a calculated thickness of 50.37"). It should also be pointed out, that in both radiance images, 

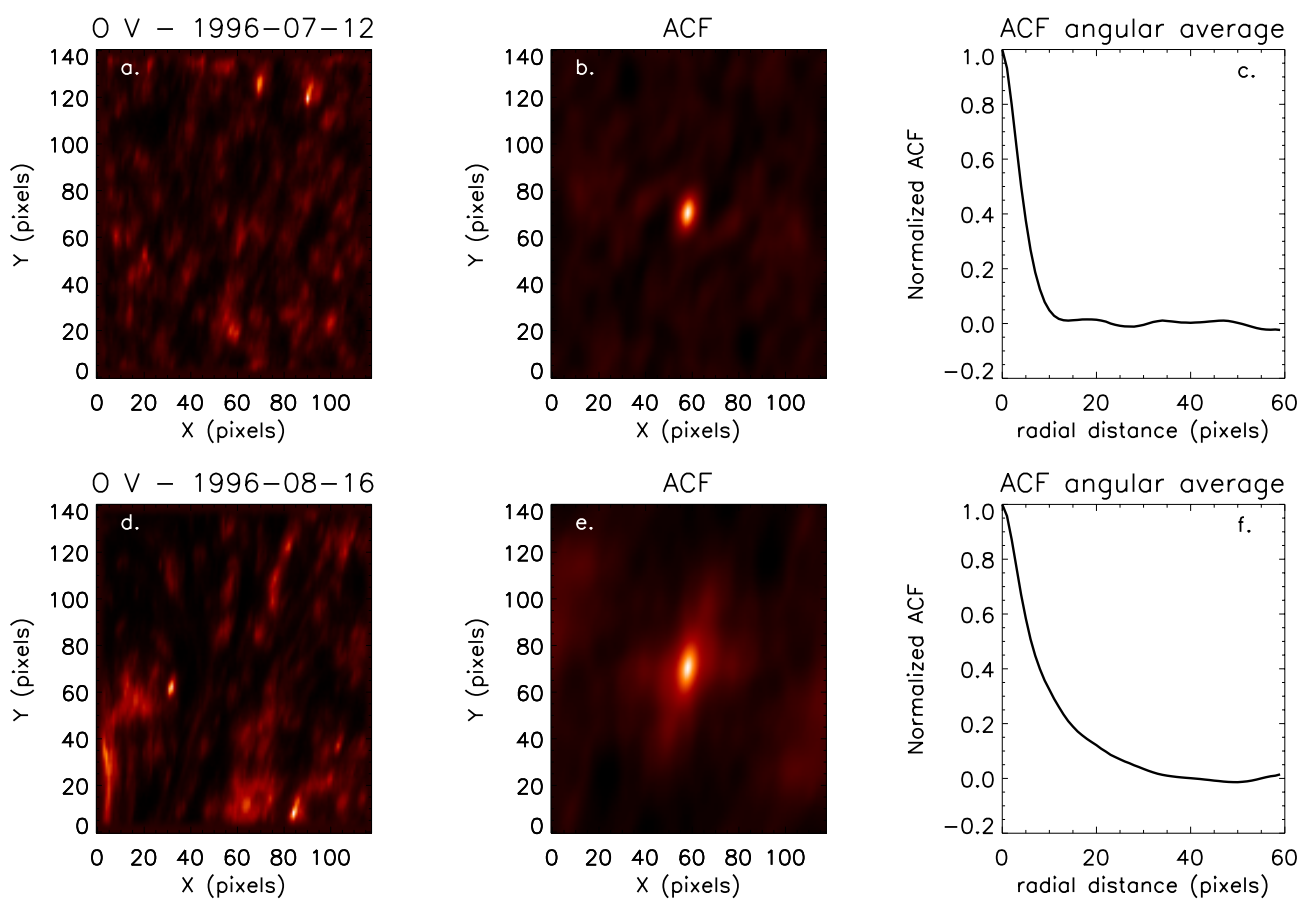

Figure 4.19: From left to right: O v intensity images taken on $1996 \mathrm{Jul} 12$ and 1996 Aug 16, corresponding to a quiet Sun condition $(a)$ and the appearance of an AR $(d)$, their associated autocorrelation functions ( $b$ and $e$ ) and the azimuthal average of the ACFs along the radial directions ( $c$ and $f$ ).

the network and AR structures are slightly oriented along the $y$ axes. Therefore, the central peaks in their corresponding ACFs (panels $b$ and $e$ ), are not circular, but elongated along the $y$ direction (and slightly tilted toward the diagonal in panel $e$ ). The effect is much larger in panel $e$ ), when an AR appears in the field-of-view. In case of quiet Sun conditions, although the ACF does not seem to be perfectly isotropic, it was still decided to calculate the angular average, since no preferential direction can be thought of when estimating the HWHM of the ACF central peak. Hence, the angular average of the ACF, as pointed out earlier, results in a maximum network size.

The size of the network structures was calculated for all intensity images during the two solar minima. For estimating the precise network size, the spatial resolution is a determinant factor. However, as discussed in Sec. 4.2, up to $3 \times 3$ binning there is no significant loss of spatial resolution. In fact, the average thickness is only slightly larger when measured from binned images (see Table 4.1).

The network size values are plotted in Fig. 4.20. Note that the network thickness in the figure is given in pixels. Multiplying the thickness values in pixels by the maximum pixel size of the instrument ( $2^{\prime \prime}$, in solar $x$ direction) for binsize 1 , and by $3 \times 2^{\prime \prime}$ for binsize 3 , returns the maximum network thickness in " unit. The average values of the network thickness over solar minima $(\bar{t})$, together with their associated standard deviations $\sigma$ and standard error of the means $\operatorname{Err}_{\bar{t}}$, are shown in Table 4.1. The ratio between the average network thickness from minimum 23 to those of minimum $22\left(r_{\bar{t}}=\bar{t}_{23} / \bar{t}_{22}\right)$ and their standard deviations and errors, are also given in Table 4.2.

The calculated size of the network structures are in very good agreement with those 
$\mathrm{He}$ I

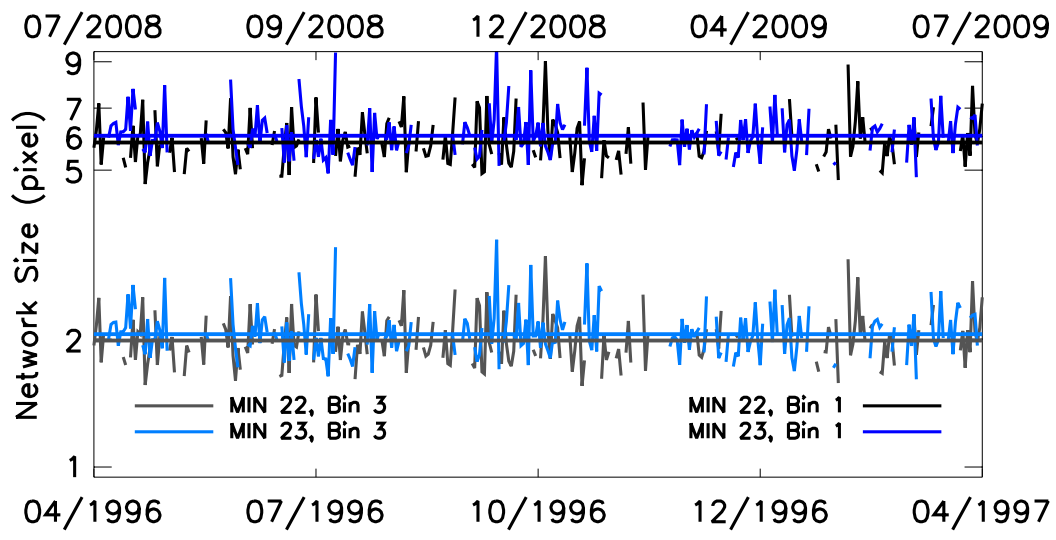

$\mathrm{OV}$

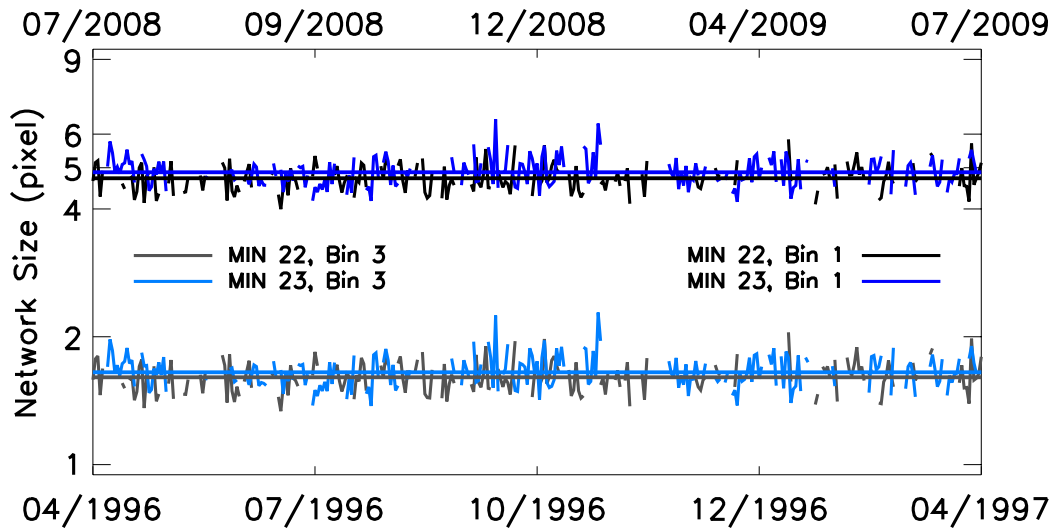

Figure 4.20: Comparison of the average network thickness in solar minima 22 (plotted in black and grey for binsize 1 and 3, respectively) and 23 (plotted in dark and light blue for binsize 1 and 3, respectively), and for the $\mathrm{He}_{\mathrm{I}}$ (top) and $\mathrm{O} \mathrm{v}$ (bottom) spectral lines. The horizontal solid lines indicate the mean network size over each minimum.

found by Patsourakos et al. (1999). They also applied the same method to CDS/NIS data and found network thickness of $12^{\prime \prime}$ and 9.5" for the He I and $\mathrm{O} v$ lines, respectively.

The network size values is approximately $2 \%-3 \%$ larger when estimated from the binned images (with binsize 3) than those calculated from the non-binned images. In addition, comparing the two solar minima and regardless of the choice of the binsize value, the average network thickness in both spectral lines is larger in minimum 23. Considering the amount of these increases and their uncertainties (given in Table 4.2), the increases in the average network size of both spectral lines are statistically identical.

The increase in the size of the network structure as seen here, may have instrumental origins. As already mentioned, one factor that can drastically affect the outcome of this analysis is the PSF of the instrument. However, the fact that even the binned data show a similar amount of increase during minimum 23 as seen in non-binned data, may rule out the effect of PSF (due to the fact that the average width of the lanes is anyway larger than the instrument spatial resolution). Note that the same analysis using the images, binned over $4 \times 4$ pixels, resulted in very similar increase. Although it may come to mind that an 
Table 4.1: Average network thickness (given in " units).

\begin{tabular}{c|cc|ccc}
\hline Sp. line & Bin. & Min. & Net.thick. & $\operatorname{SD}_{\bar{t}}(\sigma)$ & $\operatorname{Err}_{\bar{t}}$ \\
\hline \multirow{3}{*}{$\mathrm{He}_{\mathrm{I}}$} & 1 & 22 & 11.62 & 1.40 & 0.10 \\
& & 23 & 12.05 & 1.32 & 0.08 \\
\cline { 2 - 6 } & 3 & 22 & 11.91 & 1.53 & 0.11 \\
& & 23 & 12.33 & 1.45 & 0.09 \\
\hline \hline \multirow{3}{*}{$\mathrm{O}_{\mathrm{v}}$} & 1 & 22 & 9.44 & 0.68 & 0.05 \\
& & 23 & 9.76 & 0.74 & 0.05 \\
\cline { 2 - 6 } & 3 & 22 & 9.63 & 0.80 & 0.05 \\
& & 23 & 9.90 & 0.83 & 0.06 \\
\hline
\end{tabular}

increase in the size of the network structure should lead to larger CRs over minimum 23, it must be noted that the average radiance of the network does not necessarily increase by the size of the network structure.

Table 4.2: Ratio between average network thickness values of solar minimum 23 to those of minimum 22 .

\begin{tabular}{cc|ccc}
\hline Sp. line & Bin. & $r_{\bar{t}}$ & $\mathrm{SD}_{r_{\bar{t}}}$ & Err $_{r_{\bar{t}}}$ \\
\hline $\mathrm{He}_{\mathrm{I}}$ & 1 & 1.037 & 0.169 & 0.011 \\
& 3 & 1.035 & 0.180 & 0.012 \\
\hline $\mathrm{O}_{\mathrm{v}}$ & 1 & 1.033 & 0.108 & 0.007 \\
& 3 & 1.028 & 0.121 & 0.008 \\
\hline
\end{tabular}

\subsection{Periodic appearance of $A R$ in minimum 22}

As it was shown in the previous section, the output of the autocorrelation analysis used to estimate the width of the network lane, increases significantly with the appearance of an active region in the intensity image (see Fig. 4.19). In fact, the existence of unusually bright structures (i.e., ARs) in the field-of-view of the instrument, often results in the enhancement of all the parameters discussed so far, such as the CRs and the parameters of the lognormal fits. In Sec. 3.5, it was explained that the raster scans with such bright structures were visually selected and excluded from the analyses in order to have a consistent and uniform data set of the quiet Sun observations. In Fig. 4.21, the values of the network thickness and the shape parameters of all these excluded raster scans (indicated by red plus signs), together with those of the quiet Sun observations (marked by black plus signs), are plotted over minimum 22. The unfiltered data shows an approximately 28 -day periodicity in the value of the shape parameter (mostly) and in other parameters derived in our analysis. This periodicity lasts for much of minimum 22 and is visible in the data taken in the southern hemisphere. 


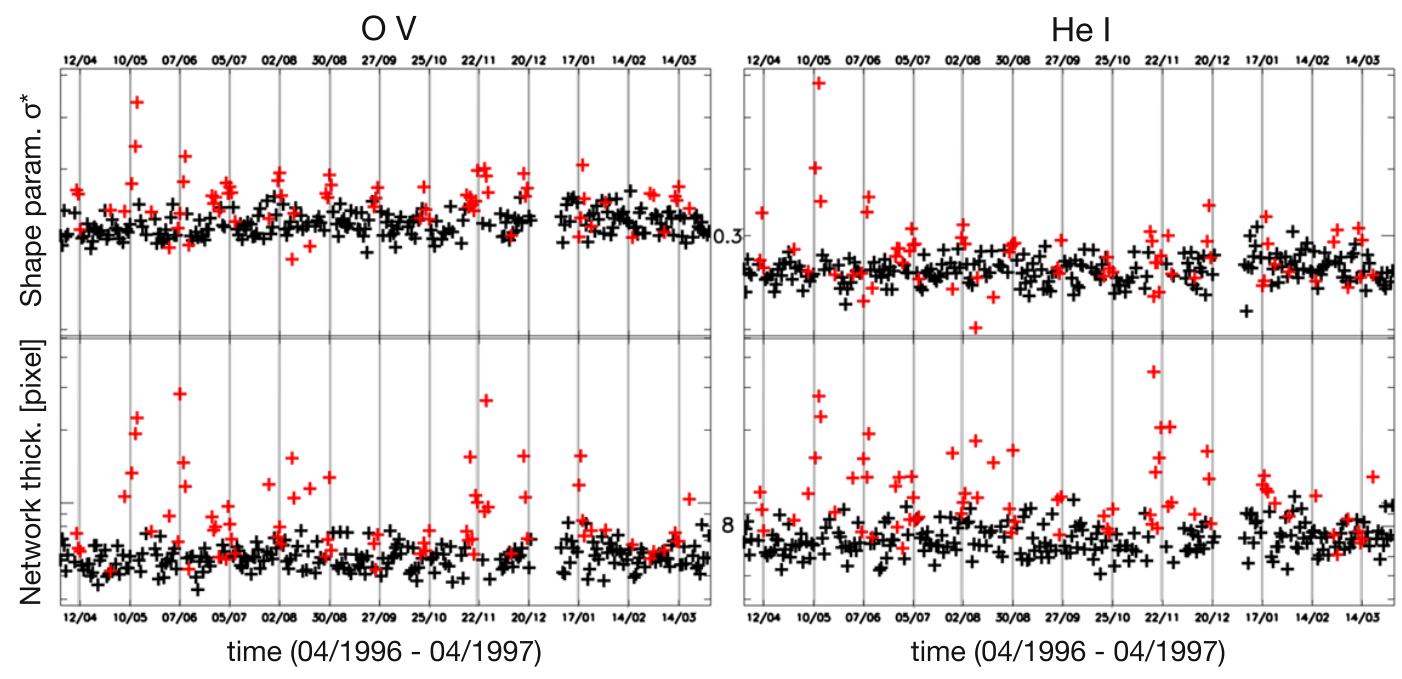

Figure 4.21: Periodic appearance of ARs and its effect on the enhancement of the shape parameters $\sigma^{*}$ (top panels) and the network thickness (bottom panels). The red and black plus signs correspond to the AR and quiet Sun conditions, respectively. The vertical lines roughly indicate the time of the enhancements during minimum 22.

The periodicity in the $5 \% \mathrm{CR}$ and the shape parameter $\sigma^{*}$ of the $\mathrm{O} v$ line, was studied over three other regions of the solar disc in addition to the disc center. These region are indicated on the right side of Fig. 4.22 and correspond to the raster scans no. 2, 4, 5 and 6 of the CDS Synop study (see Sec. 3.1), covering solar disc regions between 360" to 580", $-120^{\prime \prime}$ to $120^{\prime \prime},-360^{\prime \prime}$ to $-120^{\prime \prime}$ and $-580^{\prime \prime}$ to $-360^{\prime \prime}$, respectively. The missing points of the data sets were filled by interpolation and the power spectra were produced using the FFT (Fig. 4.22).

In minimum 22, both parameters show clear cyclic patterns with a period of approximately 28 days (with harmonics of 14, 9 and 6.8 days), in the southern hemisphere of the Sun (regions no. 5 and 6). The periodicity in the central region (no. 4), although less significant, is still recognisable, especially when looking at the power spectrum of the shape parameter. In the northern hemisphere (region no. 2), the periodicity disappears almost entirely. The situation is very different in minimum 23 , during which, none of the parameters show any sign of periodicity (region no. 5 was not analysed during minimum 23 ). This is related to the very low number of sunspots (and consequently ARs) during the weak minimum between solar cycles 23 and 24 .

In Fig 4.23, the power spectra of the FFT of the 5\% CRs over minimum 22 and in rasters no. 4, 5 and 6, are plotted at top of each other. The black line in the figure, corresponding to the central rasters, has a sharp peak at 27.8 days. In the southern hemisphere (rasters no. 5 and 6, plotted in blue and violet, respectively), the peak becomes broader. The power spectrum in rasters no. 6, almost show a double-peak at 25.8 and 32.9 days. This is due to the larger solar surface coverage within the CDS field-of-view $\left(240^{\prime \prime} \times 240^{\prime \prime}\right)$, such that the entering and the exit of long-lasting ARs into and out of the field-of-view of the instrument, produce two peaks that are more distant each other..

A study by Giordano and Mancuso (2008) revealed that the radiance of the $\mathrm{O}$ vi line at $103.2 \mathrm{~nm}$ and the H I Ly $\alpha$ spectral line at $121.6 \mathrm{~nm}$ spectral lines, measured from May 1996 
to May 1997, exhibit cyclic variation with an average period of $28.16 \pm 0.2$ days at midlatitudes in the southern hemisphere. Such periodicity was also reported by Knaack et al. (2005), who found "quasi-periodic oscillations" in the magnetic field of the photosphere in the southern hemisphere with a period of 28.1 days. These studies, as well the results from the FFT analyses mentioned above, clearly show a much magnetically active conditions during solar minimum 22 close to the disc-center. 


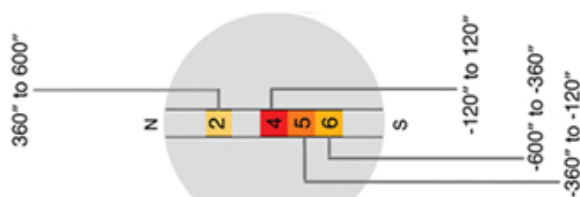

षิ

苯

预

츨

스를

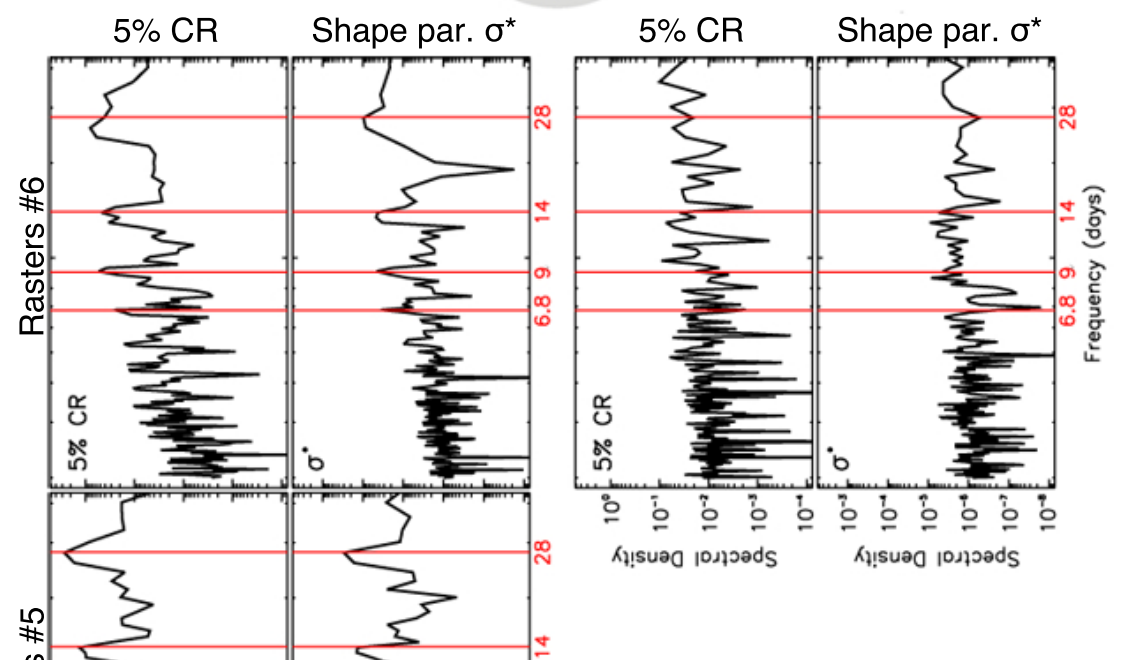

త్

을

$\Xi \Xi$

:气

층

क స్ㅜㅇ

莒过

击

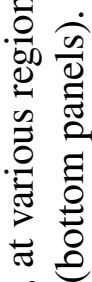

* त

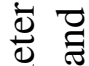
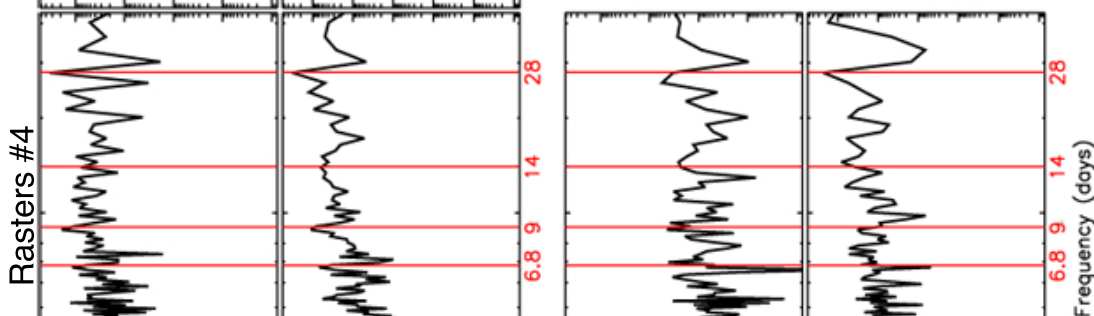

증

ีำ

艺

के

즈

뜰

识

>

0 in

䟚

$\Xi$

永

:

के

-

ซิ $\cong$

ते

궁응 음

0 o

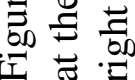




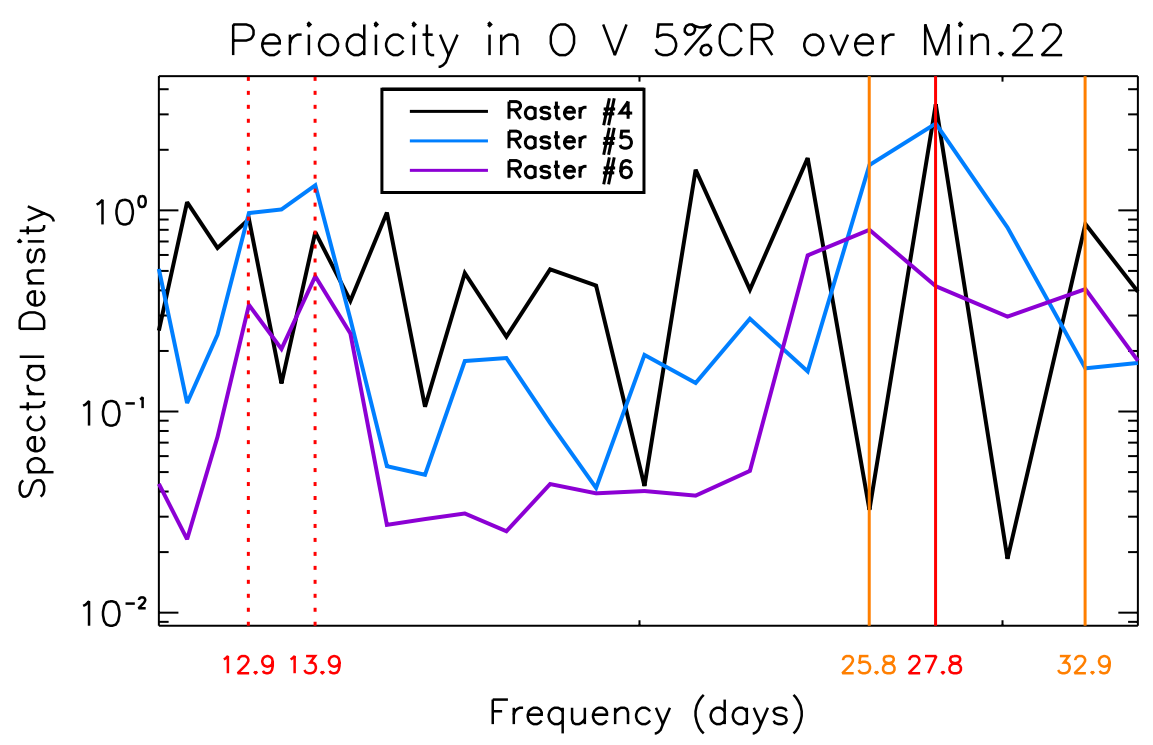

Figure 4.23: Power spectra of the FFT of the Ov 5\% CR at regions no. 4, 5 and 6 (indicated by black, blue and violet solid lines, respectively), during the solar minimum 22 . The red solid vertical line corresponds to the peak of the spectral densities of the regions no. 4 and 5 , while the peaks of the power spectrum in region no. 6 is indicated by the orange solid vertical lines. The red dotted vertical lines correspond to the secondary peak (second harmonic of the synodic periodicity close to 13-day), for power spectra of regions no. 5 and 6. 



\section{Discussion and conclusion}

In this work, the variation in the daily average intensities and statistical parameters of the radiance distributions of two spectral lines from the chromosphere and transition region (He I and OV, respectively), were investigated throughout the solar cycle 23, with emphasis on the solar minima before and after this cycle.

The degradation of the CDS instrument's photometric sensitivity (see Sec. 4.1) makes the absolute intensity of the spectral lines unreliable for long-term investigations of subtle solar changes, such as the difference in brightness between the minima following cycles 22 and 23. The parametrization of the radiance distribution in the quiet Sun and the study of those parameters that are independent of the absolute intensity, is a possible alternative that was considered during this thesis work. For this purpose, two contrast ratios (CRs) were defined: the ratio between the mean intensity of the brightest and darkest $5 \%$ and $10 \%$ of the intensity images (see Fig. 4.4). This way, the effect of correction and calibration factors were cancelled out (to the extend possible). The intensity distributions were also fitted by lognormal distribution functions and the fit parameters were extracted and a comparative study of all these parameters over the last two solar minima was carried out to investigate the variations in the radiance distribution. The images with bright structures in the field of view were excluded from the analyses to concentrate only on the quiet Sun area. The exclusion procedure was based either on visual inspection (Fig. 3.7) or on the goodness of the lognormal fits to the intensity histograms (Fig. 4.14). In addition, the NIS raster scans were down-sampled (binned) prior to any further analyses to increase the signal to noise ratio over darker areas and, thus, compensate for the effect of reduced sensitivity of the instrument over the 11 years separating the two minima (see Sec. 4.2.1). This does not effectively reduce the instrument spatial resolution up to binsize 3 , as the instrument PSF is almost three times larger than the CDS actual pixel size (see Sec. 3.4). Hence, binsizes 3 and 4 ( $3 \times 3$ and $4 \times 4$, respectively) are found to be the optimal values for down-sampling in order to have maximum SNR and minimum loss of spatial resolution. This is in agreement with previous work by Brković et al. (2000).

The CRs in the $\mathrm{O} v$ line exhibit a measurable drop over solar minimum 23. The magnitude of the reduction, however, is sensitive to the data exclusion methods. From Fig. 4.6, when considering binsizes 3 and 4, exclusion of $30 \%$ of the images by visual inspection reveals a drop of around $4 \%$ in the minimum-to-minimum trend. The drop is almost doubled when the exclusion is based on the $\chi^{2}$ of the lognormal fits, displayed in Fig. 4.17. The latter figure shows that no substantial changes occur when rejection levels larger than $10 \%$ are used.

The CRs, are in principle a measure of contrast between the network and internetwork radiances. Moreover, the $\mathrm{O} v$ radiance distributions undergo a shift from a skewed form to a more symmetric shape during the very quiet minimum of cycle 23 . Such a variation of the 
distribution manifests itself in terms of a decrease in the shape and skewness parameters illustrated in Fig. 4.16, where the skewness of the intensity distribution of the $\mathrm{O} v$ line shows a drop of the $3.5 \%$ over solar activity minimum 23 . The smaller dispersion in the $\mathrm{O} v$ intensity distribution, as quantified by the coefficient of variation (panel $c$ ) of Fig. 4.16), during activity minimum 23 suggests that either the thermal structure of the internetwork has changed between the minima following cycles 22 and 23 , or the area covered by the network or the brightness of the network. The latter two possible explanations relate to the amount of magnetic flux. A lower magnetic flux is also found to be the cause of the lower TSI during the minimum of cycle 23 (Ball et al. 2012; Yeo et al. 2014).

The studies carried out by Fröhlich (2011) and Tripathy et al. (2010) show also a drop in the $10.7 \mathrm{~cm}$ flux up to $4 \%$ over minimum 23 with respect to the previous minimum. Note that the $10.7 \mathrm{~cm}$ radio flux is a global solar activity factor and it is not trivial to compare it directly with our results, which are entirely based on local observations of the solar disc-center.

The $\mathrm{He}$ I spectral line, in contrast to the $\mathrm{O} v$ line, shows no measurable minimum-tominimum variations. This is evident when looking at the CRs in Figs. 4.6 and 4.17. Both $5 \%$ and $10 \%$ CRs at binsizes 3 and 4 are very similar in the last two solar minima and their minimum-to-minimum ratios are within $1 \%$ of the unity line. It is also interesting to note that the $\mathrm{He}$ I line is less sensitive to the exclusion of data due to the presence of magnetic activity. Excluding only $10 \%$ of the images with the most obvious bright structures, is sufficient for the total brightness not to be affected by the remaining traces of activity. This is because the contrast between network and internetwork is larger in the transition region $\mathrm{O} v$ line than in the chromespheric He I line.

These findings are compatible with those of Livingston et al. (2010), who found that the chromospheric $\mathrm{Ca}$ II $\mathrm{K}$ line, measured over solar disc-center, is constant (within 1\%) between the last three solar activity minima. This is important, since the $\mathrm{Ca}$ II $\mathrm{K}$ index is usually regarded to display a relatively tight relationship with the photospheric magnetic field (Skumanich et al. 1975; Schrijver et al. 1989; Lukianova 2009). The He I and Ca II K results imply a certain level of decoupling between the chromospheric radiation and the photospheric field, at least at very low level of activity.

In a recent study by Andretta and Del Zanna (2014), the authors also analysed the radiance distribution over solar cycle 23 by investigating the full-disc and central meridian observations of the CDS studies USUN and SYNOP, respectively. They concluded that the quiet Sun radiance has undergone no significant changes during solar cycle 23, regardless of which spectral line is considered. Although the same conclusion was obtained for the $\mathrm{He}$ I line, a minimum-to-minimum variation in the radiance distribution of the $\mathrm{O} v$ line was found instead during this thesis work. On the other hand, the values they found as the average widths of the lognormal fits over the full cycle are in good agreements with our findings (when rescaled from natural logarithm to normal logarithm). The average width values for histograms in the $\mathrm{He} \mathrm{I}$ and $\mathrm{O} \mathrm{v}$ lines were found to be equal to $0.18 \pm 0.01 \mathrm{dex}$ and $0.22 \pm 0.02$ dex, respectively. The values reported in the study by Andretta and Del Zanna (2014) are $0.16 \pm 0.015$ dex for the He I line and $0.19 \pm 0.02$ dex for the $O v$ line. The small difference is likely due to the specific fitting approach applied by (Andretta and Del Zanna 2014), in which only the peak of the histograms, corresponding to the internetwork area, are fitted and the contribution of the brighter network has been ignored. This is also the likely explanation of the lack of detection of variations in the $\mathrm{O} V$ radiance claimed by 
these authors..

In the model introduced by Dowdy et al. (1986), a two component mechanism is assumed to be responsible for producing the emission in the transition region (TR): a down-flow of the heat within the magnetic funnels which expand upward throughout the network structures connecting to the corona, and the internal heating of the gas inside low-lying small loops, which are magnetically insulated from the corona. Each of these two components connects the radiance formation in the TR to the chromosphere and corona.

The difference in behaviour of the chromospheric and TR lines found here may have different origins. One possibility is that the $\mathrm{He}_{\mathrm{I}}$ line reacts less strongly to the presence of a magnetic field than the $\mathrm{O} v$ line. Note that, as it was shown in sec. 4.6, more active regions were present close and at the solar disc-center during solar minimum 22 . The more magnetically active conditions at solar minimum 22 may have enhanced the network brightness of $\mathrm{O} v$ line, leading to more skewed shape of the radiance distributions and larger CRs.

Another possibility is that the heating of the quiet chromosphere is much more strongly determined by acoustic waves than the TR. Hence in He I what is basically seen is basal flux in the very quiet Sun, while in TR lines a more magnetically dominated heating mechanism is active. The former does not change from minimum to minimum, while the latter does. 



\section{Outlook}

Following the analyses of CDS data over the last two solar minima, it was decided to apply the same analyses to the data obtained with the Extreme-ultraviolet Imaging Telescope (EIT; Delaboudinière et al. 1995), to see if and how the results differ from those already obtained from CDS data. Using EIT data also makes it possible to study the radiance variation in the corona.

The EIT data were chosen corresponding to already processed CDS data, covering two 1-year time-spans over the solar minima 22 and 23. The full-disc images from EIT were cropped, such to be consistent with the spatial coverage of the CDS field-of-view (by taking into account the effect of the solar rotation). The EIT images in He II at $30.4 \mathrm{~nm}$ and Fe IX-X at $17.1 \mathrm{~nm}$ (corresponding to chromospheric network and corona/TR boundary, respectively) were chosen to be analysed, as being complementary to the CDS observations.

The EIT images were binned over $2 \times 2$ pixels, to make the spatial resolution of the EIT images comparable to that of the CDS intensity images after being binned over $3 \times 3$ pixels. Note that, the pixel size of EIT and CDS are $2.59^{\prime \prime} \times 2.59^{\prime \prime}$ and $2^{\prime \prime} \times 1.67^{\prime \prime}$, respectively. In addition, in analogy to the data exclusion carried out for the CDS analyses, approximately $30 \%$ of the EIT images, in which an AR or any unusually bright features appear in the field-of-view, were excluded from the analyses. Finally, the same set of statistical parameters that were defined and calculated for analysing the CDS intensity intensity (CRs and lognormal fit parameters), were produced using EIT images.

The 5\% and 10\% CRs of EIT images for two binsize values of 1 (no binning) and 2 are illustrated in Figs. 6.1 and 6.2 for the He II and Fe IX-X spectral lines, respectively. In all plots, the average CRs show a drop during minimum 23. This is in agreement with the results from CDS observations. However, when comparing the minimum-to-minimum ratio of the CRs from both instruments, the amount of drop is much larger in the case of EIT observations. This comparison is depicted in Fig. 6.3. The blue and the black lines in the figure correspond to the ratios of CRs for CDS spectral lines He I and O v, respectively, as already shown in Fig. 4.6. The red and orange bars indicate the mean and the standard errors of the EIT He II at $30.4 \mathrm{~nm}$ and Fe IX-X at $17.1 \mathrm{~nm}$ CRs spectral lines, respectively, for binsize values of 1 and 2 (roughly comparable to the CDS binsize values of 2 and 3). Abbreviations v1 to v3 denote different versions of data exclusion corresponding to 10 to 30 percent CDS data exclusion.

The lognormal fit parameters of the EIT intensity histograms, also exhibit remarkable drops during minimum 23. The ratio between the fit parameters of EIT are plotted alongside those of CDS in Fig. 6.4. The drop in the fit parameters of the EIT intensity distributions at $17.1 \mathrm{~nm}$ seem to be similar to those of the $\mathrm{O} v$ line observed by CDS, when comparing the binsize 1 of EIT with the binsize 2 of CDS. However, as soon as the EIT images are being binned, the difference becomes very large. The fit parameters of EIT He II line are less 


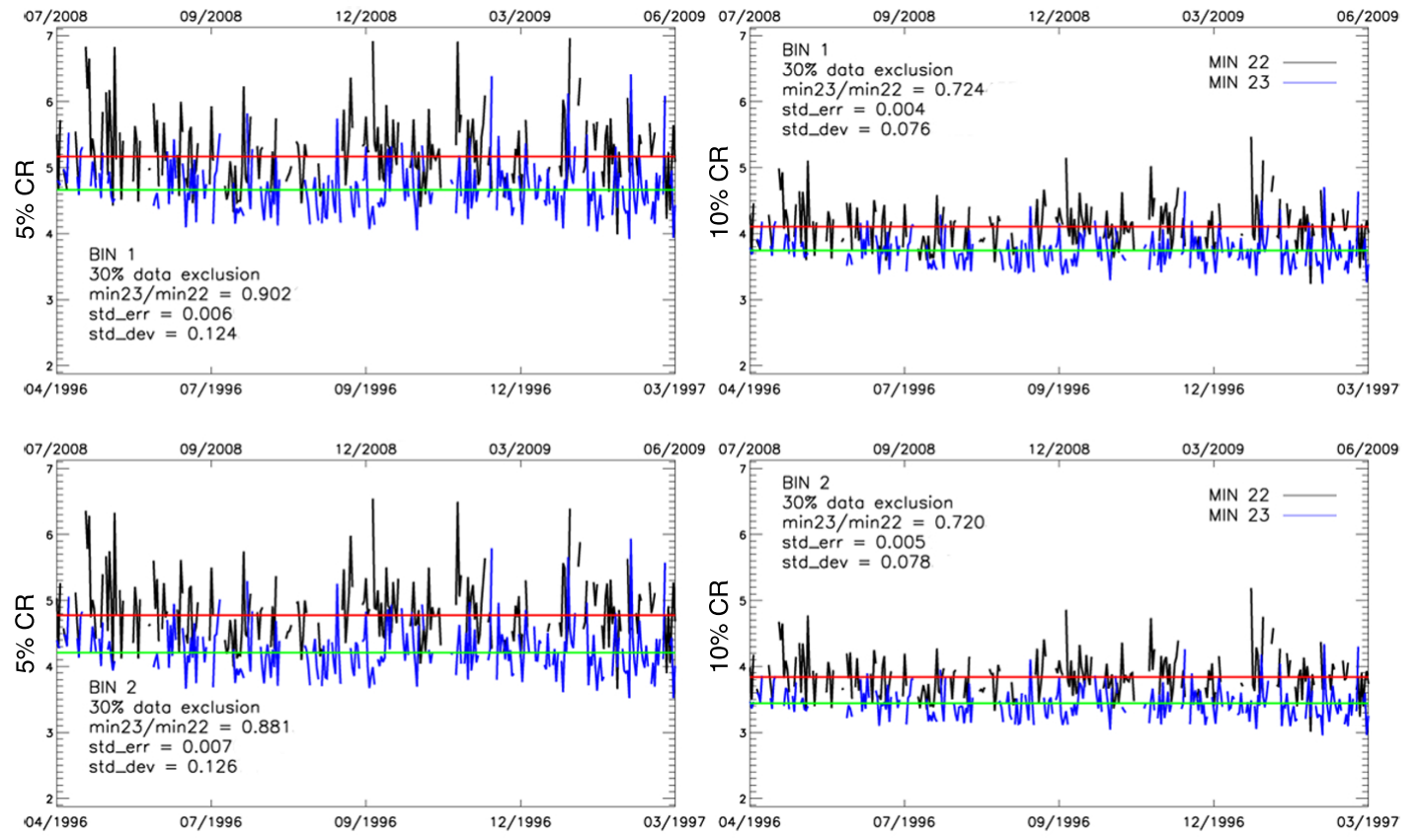

Figure 6.1: EIT 5\% (left panel) and 10\% (right panel) CRs for binsizes 1 (top) and 2 (bottom) calculated for the He II spectral line at $30.4 \mathrm{~nm}$. The red and the green horizontal lines correspond to the average value over minimum 22 (black lines) and 23 (blue lines), respectively.

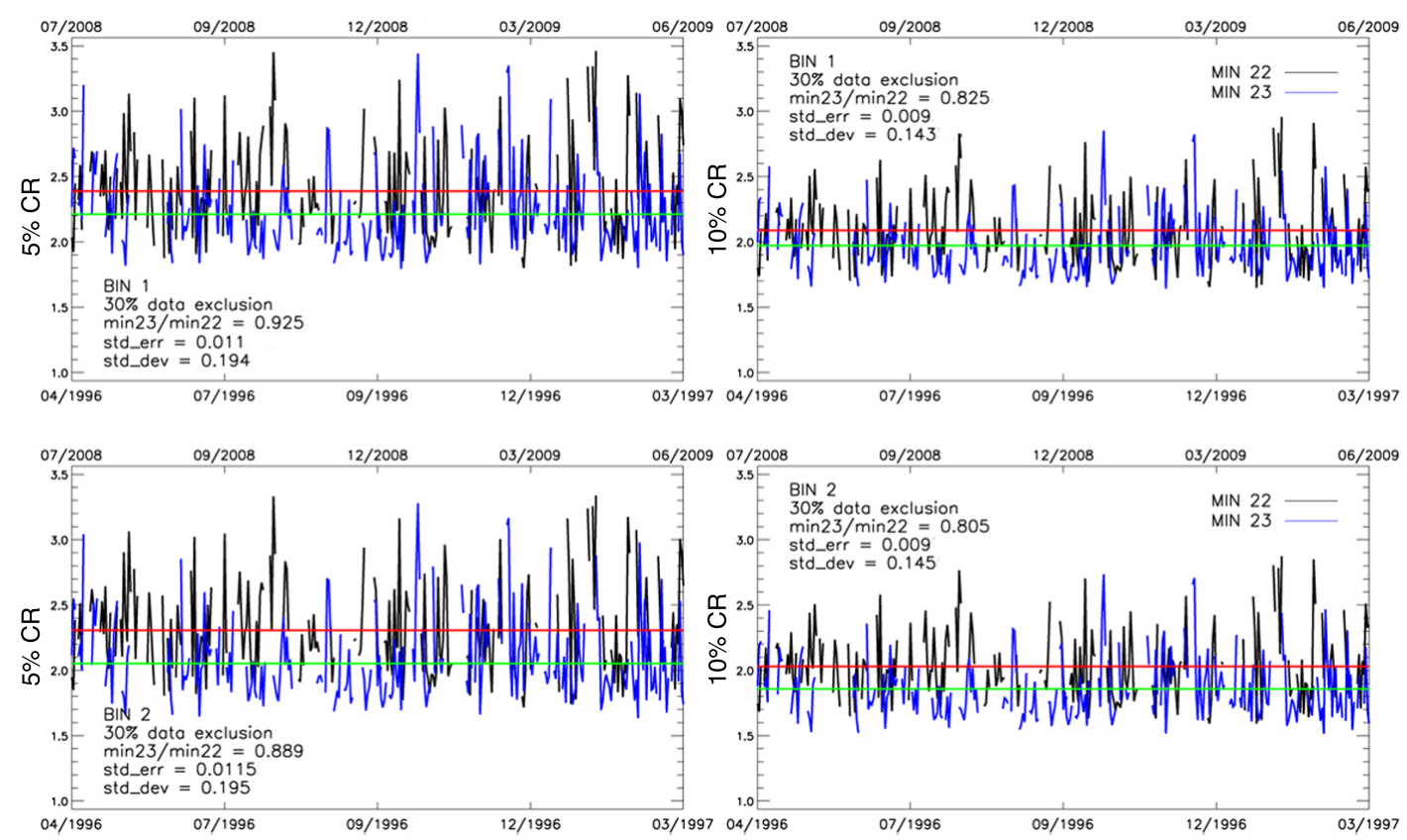

Figure 6.2: EIT 5\% (left panel) and 10\% (right panel) CRs for binsizes 1 (top) and 2 (bottom) calculated for the FeIX-X spectral line at $17.1 \mathrm{~nm}$. The red and the green horizontal lines correspond to the average value over minimum 22 (black lines) and 23 (blue lines), respectively. 

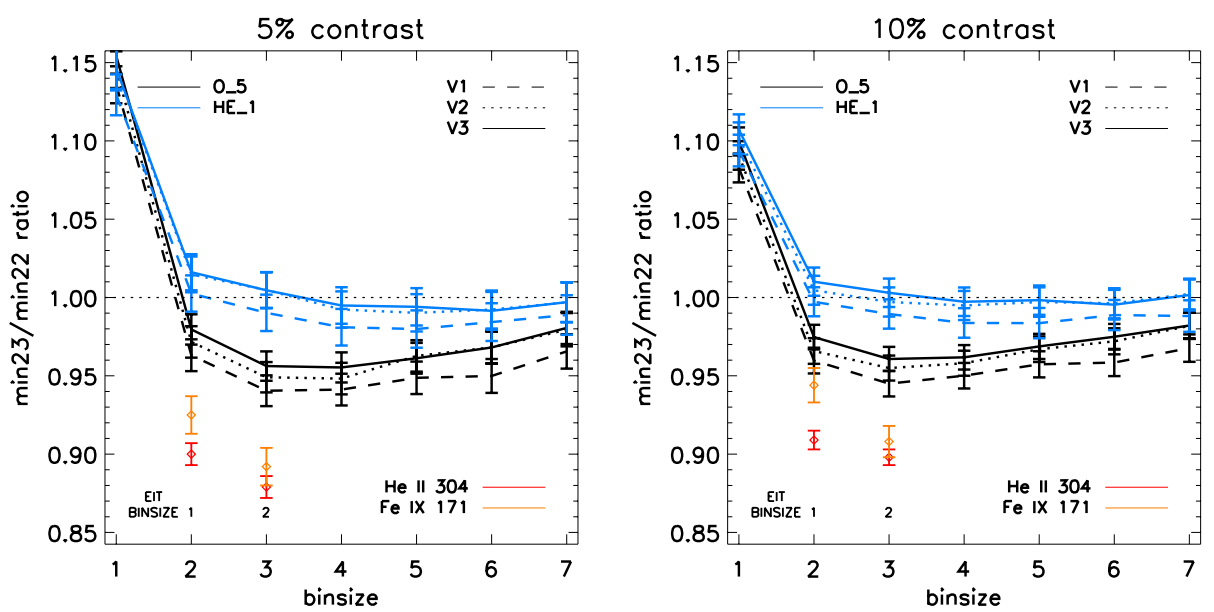

Figure 6.3: Comparison between the minimum-to-minimum ratios of the CDS and EIT CRs. The dashed, dotted and solid lines are corresponding to the three versions of data exclusion, corresponding to $10 \%, 20 \%$ and $30 \%$ data exclusion due to existence of bright features inside the field-of-view. The error bars represent the $1 \sigma$ error in the ratios. The orange and the red diamonds and their associated errors bars, correspond to the minimumto-minimum ratios of the EIT CRs in He II and Fe IX-X spectral lines, respectively. The black horizontal dotted line indicates the unity line. As explained in the text, spatial resolution of the unbinned EIT data (labelled by EIT binsize 1) is comparable with the CDS data after being binned over $2 \times 2$ pixels (binsize 2). Similarly, EIT binsize 2 is comparable to CDS binsize 3 .
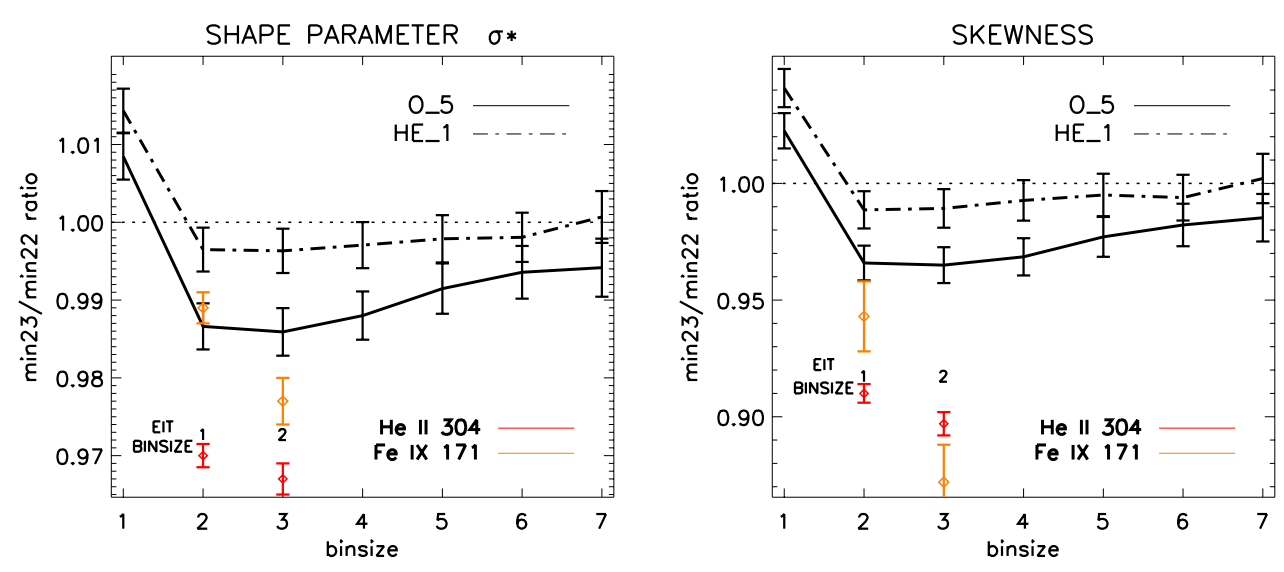

Figure 6.4: Comparison between the minimum-to-minimum ratios of the CDS and EIT lognormal fit parameters. The dash-dotted and the solid lines are corresponding to the ratio of the average values of the shape parameter (left) and the skewness (right) during solar minimum 23 to those during solar minimum 22 for the CDS He I and $\mathrm{O}$ v spectral lines, respectively. The error bars represent the $1 \sigma$ error in the ratios. The orange and the red diamonds and their associated errors bars, correspond to the minimum-to-minimum ratios of the same parameters for the EIT He II and Fe IX-X spectral lines, respectively. The black horizontal dotted line indicates the unity line. The EIT binsizes of 1 and 2 are compared to the CDS binsizes of 2 and 3, respectively. 
sensitive to the binning, but it is not possible to compare them with any of the CDS lines.

The calibration of EIT data is a very complex procedure. The most important issue with EIT data is the presence of the stray light (Auchère et al. 2001), which is a source of huge uncertainty in all EIT observations. The excess radiation at the dark regions of the EIT images due to stray-light, can significantly change the contrast ratios and the shape of the radiance distributions. Therefore, to have a better estimation of the drops in CRs and the fit parameters and a reliable interpretation of these changes, the exact amount of stray-light inside the instrument must be known. This analysis, currently in progress, is crucial at determining the correct radiance distributions. The latter are crucial to extend the study to coronal temperatures.

2015arXiv150705786S 


\section{Bibliography}

Andretta, V., Del Zanna, G., 2014, The EUV spectrum of the Sun: SOHO CDS NIS radiances during solar cycle 23, ArXiv e-prints, 1401.7570

Andretta, V., Del Zanna, G., Jordan, S. D., 2003, The EUV helium spectrum in the quiet Sun: A by-product of coronal emission?, A\&A, 400, 737-752

Aschwanden, M. J., 2005, Physics of the Solar Corona. An Introduction with Problems and Solutions (2nd edition)

Aschwanden, M. J., 2008, An Observational Test That Disproves Coronal Nanoflare Heating Models, ApJ, 672, L135-L138

Aschwanden, M. J., Charbonneau, P., 2002, Effects of Temperature Bias on Nanoflare Statistics, ApJ, 566, L59-L62

Aschwanden, M. J., Parnell, C. E., 2002, Nanoflare Statistics from First Principles: Fractal Geometry and Temperature Synthesis, ApJ, 572, 1048-1071

Athay, R. G. (Ed.), 1976, The solar chromosphere and corona: Quiet sun, vol. 53 of Astrophysics and Space Science Library

Athay, R. G., 1982, Responses of transition region models to magnetic field geometry and downflow velocities, ApJ, 263, 982-986

Athay, R. G., 1988, The origin of solar He II radiation, ApJ, 329, 482-492

Athay, R. G., 1990, A model of the solar chromosphere-corona transition region based on classical thermal conduction, ApJ, 362, 364-369

Athay, R. G., Thomas, R. N., 1961, Physics of the solar chromosphere

Auchère, F., Hassler, D. M., Slater, D. C., Woods, T. N., 2001, SWRI/LASP Sounding Rocket Inter-Calibration With The Eit Instrument On Board SOHO, Sol. Phys., 202, 269-280

Avrett, E. H., 1981, Reference model atmosphere calculation - The Sunspot sunspot model, in The Physics of Sunspots, (Eds.) L. E. Cram, J. H. Thomas, pp. 235-255

Avrett, E. H., Loeser, R., 1992, The PANDORA Atmosphere Program (Invited Review), in Cool Stars, Stellar Systems, and the Sun, (Eds.) M. S. Giampapa, J. A. Bookbinder, vol. 26 of Astronomical Society of the Pacific Conference Series, p. 489 
Babcock, H. W., 1961, The Topology of the Sun's Magnetic Field and the 22-YEAR Cycle., ApJ, 133, 572

Ball, W. T., Unruh, Y. C., Krivova, N. A., Solanki, S., Wenzler, T., Mortlock, D. J., Jaffe, A. H., 2012, Reconstruction of total solar irradiance 1974-2009, A\&A, 541, A27, 1202.3554

Bazarghan, M., Safari, H., Innes, D. E., Karami, E., Solanki, S. K., 2008, A nanoflare model for active region radiance: application of artificial neural networks, A\&A, 492, L13-L16, 0808 . 3925

Beer, J., Vonmoos, M., Muscheler, R., 2006, Solar Variability Over the Past Several Millennia, Space Sci. Rev., 125, 67-79

Bello González, N., Franz, M., Martínez Pillet, V., Bonet, J. A., Solanki, S. K., del Toro Iniesta, J. C., Schmidt, W., Gandorfer, A., Domingo, V., Barthol, P., Berkefeld, T., Knölker, M., 2010, Detection of Large Acoustic Energy Flux in the Solar Atmosphere, ApJ, 723, L134-L138, 1009 . 4795

Benz, A. O., Krucker, S., 1998, Heating Events in the Quiet Solar Corona, Sol. Phys., 182, 349-363

Bercik, D. J., Fisher, G. H., Johns-Krull, C. M., Abbett, W. P., 2005, Convective Dynamos and the Minimum X-Ray Flux in Main-Sequence Stars, ApJ, 631, 529-539, astro-ph/ 0506027

Berger, T. E., Schrijver, C. J., Shine, R. A., Tarbell, T. D., Title, A. M., Scharmer, G., 1995, New Observations of Subarcsecond Photospheric Bright Points, ApJ, 454, 531

Biermann, L., 1946, Zur Deutung der chromosphärischen Turbulenz und des Exzesses der UV-Strahlung der Sonne, Naturwissenschaften, 33, 118-119

Bogdan, T. J., 1992, Flux tube sizes and temporal evolution, in Electromechanical Coupling of the Solar Atmosphere, (Eds.) D. S. Spicer, P. MacNeice, vol. 267 of American Institute of Physics Conference Series, pp. 1-12

Bogdan, T. J., Gilman, P. A., Lerche, I., Howard, R., 1988, Distribution of sunspot umbral areas - 1917-1982, ApJ, 327, 451-456

Bowness, R., Hood, A. W., Parnell, C. E., 2013, Coronal heating and nanoflares: current sheet formation and heating, A\&A, 560, A89

Brandenburg, A., Subramanian, K., 2005, Astrophysical magnetic fields and nonlinear dynamo theory, Phys. Rep., 417, 1-209, astro-ph/0405052

Brault, J. W., White, O. R., 1971, The Analysis and Restoration of Astronomical Data via the Fast Fourier Transform, A\&A, 13, 169

Brković, A., Rüedi, I., Solanki, S. K., Fludra, A., Harrison, R. A., Huber, M. C. E., Stenflo, J. O., Stucki, K., 2000, EUV brightness variations in the quiet Sun, A\&A, 353, 1083-1093 
Brueckner, G. E., Howard, R. A., Koomen, M. J., Korendyke, C. M., Michels, D. J., Moses, J. D., Socker, D. G., Dere, K. P., Lamy, P. L., Llebaria, A., Bout, M. V., Schwenn, R., Simnett, G. M., Bedford, D. K., Eyles, C. J., 1995, The Large Angle Spectroscopic Coronagraph (LASCO), Sol. Phys., 162, 357-402

Buchholz, B., Ulmschneider, P., Cuntz, M., 1998, Basal Heating in Main-Sequence Stars and Giants: Results from Monochromatic Acoustic Wave Models, ApJ, 494, 700-714

Büchner, J., Nikutowski, B., Otto, A., 2004, Coronal Heating by Transition Region Reconnection, in SOHO 15 Coronal Heating, (Eds.) R. W. Walsh, J. Ireland, D. Danesy, B. Fleck, vol. 575 of ESA Special Publication, p. 23

Buehler, D., Lagg, A., Solanki, S. K., 2013, Quiet Sun magnetic fields observed by Hinode: Support for a local dynamo, A\&A, 555, A33, 1307.0789

Carlsson, M., Stein, R. F., 1997, Chromospheric Dynamics - What Can Be Learnt from Numerical Simulations, in European Meeting on Solar Physics, (Eds.) G. M. Simnett, C. E. Alissandrakis, L. Vlahos, vol. 489 of Lecture Notes in Physics, Berlin Springer Verlag, p. 159

Cattaneo, F., 1999, On the Origin of Magnetic Fields in the Quiet Photosphere, ApJ, 515, L39-L42

Charbonneau, P., 2005, Dynamo Models of the Solar Cycle, Living Reviews in Solar Physics, 2, 2

Choudhuri, A. R., Chatterjee, P., Jiang, J., 2007, Predicting Solar Cycle 24 With a Solar Dynamo Model, Physical Review Letters, 98, 131103, astro-ph/0701527

Danilovic, S., Beeck, B., Pietarila, A., Schüssler, M., Solanki, S. K., Martínez Pillet, V., Bonet, J. A., del Toro Iniesta, J. C., Domingo, V., Barthol, P., Berkefeld, T., Gandorfer, A., Knölker, M., Schmidt, W., Title, A. M., 2010a, Transverse Component of the Magnetic Field in the Solar Photosphere Observed by SUNRISE, ApJ, 723, L149-L153, 1008.1535

Danilovic, S., Schüssler, M., Solanki, S. K., 2010b, Probing quiet Sun magnetism using MURaM simulations and Hinode/SP results: support for a local dynamo, A\&A, 513, $\mathrm{A} 1,1001.2183$

de Toma, G., 2012, Polar magnetic fields and coronal holes during the recent solar minima, in IAU Symposium, (Eds.) C. H. Mandrini, D. F. Webb, vol. 286 of IAU Symposium, pp. 101-112

de Wijn, A. G., Stenflo, J. O., Solanki, S. K., Tsuneta, S., 2009, Small-Scale Solar Magnetic Fields, Space Sci. Rev., 144, 275-315, 0812 . 4465

Defise, J.-M., Moses, J. D., Clette, F., 1998, In-orbit performances of the EIT instrument on board SOHO and intercalibration with the EIT Calroc Sounding Rocket program, in Missions to the Sun II, (Ed.) C. M. Korendyke, vol. 3442 of Society of Photo-Optical Instrumentation Engineers (SPIE) Conference Series, pp. 126-139 
Del Zanna, G., 1999, Extreme Ultraviolet Spectroscopy Extreme Ultraviolet Spectroscopy of the Solar Corona, Ph.D. thesis, Center for Astrophysics, University of central Lancashire

Del Zanna, G., Bromage, B. J. I., Landi, E., Landini, M., 2001, Solar EUV spectroscopic observations with SOHO/CDS. I. An in-flight calibration study, A\&A, 379, 708-734

Del Zanna, G., Andretta, V., Chamberlin, P. C., Woods, T. N., Thompson, W. T., 2010, The EUV spectrum of the Sun: long-term variations in the SOHO CDS NIS spectral responsivities, A\&A, 518, A49

Delaboudiniere, J. P., Gabriel, A. H., Artzner, G. E., Millier, F., Michels, D. J., Neupert, W. M., Gurman, J. B., 1989, The extreme ultraviolet imaging telescope on board SOHO, in X-Ray/EUV Optics for Astronomy and Microscopy, (Ed.) R. B. Hoover, vol. 1160 of Society of Photo-Optical Instrumentation Engineers (SPIE) Conference Series, pp. $518-524$

Delaboudinière, J.-P., Artzner, G. E., Brunaud, J., Gabriel, A. H., Hochedez, J. F., Millier, F., Song, X. Y., Au, B., Dere, K. P., Howard, R. A., Kreplin, R., Michels, D. J., Moses, J. D., Defise, J. M., Jamar, C., Rochus, P., Chauvineau, J. P., Marioge, J. P., Catura, R. C., Lemen, J. R., Shing, L., Stern, R. A., Gurman, J. B., Neupert, W. M., Maucherat, A., Clette, F., Cugnon, P., van Dessel, E. L., 1995, EIT: Extreme-Ultraviolet Imaging Telescope for the SOHO Mission, Sol. Phys., 162, 291-312

Dere, K. P., Moses, J. D., Delaboudinière, J.-P., Brunaud, J., Carabetian, C., Hochedez, J.-F., Song, X. Y., Catura, R. C., Clette, F., Defise, J.-M., 2000, The Preflight Photometric Calibration of the Extreme-Ultraviolet Imaging Telescope EIT, Sol. Phys., 195, 13-44

Didkovsky, L. V., Judge, D. L., Wieman, S. R., McMullin, D., 2010, Minima of Solar Cycles 22/23 and 23/24 as Seen in SOHO/CELIAS/SEM Absolute Solar EUV Flux, in SOHO-23: Understanding a Peculiar Solar Minimum, (Eds.) S. R. Cranmer, J. T. Hoeksema, J. L. Kohl, vol. 428 of Astronomical Society of the Pacific Conference Series, p. 73, 0911.0870

Dikpati, M., Gilman, P. A., 2006, Simulating and Predicting Solar Cycles Using a FluxTransport Dynamo, ApJ, 649, 498-514

Dikpati, M., de Toma, G., Gilman, P. A., 2006, Predicting the strength of solar cycle 24 using a flux-transport dynamo-based tool, Geophys. Res. Lett., 33, L05102

Domínguez Cerdeña, I., Sánchez Almeida, J., Kneer, F., 2003, Inter-network magnetic fields observed with sub-arcsec resolution, A\&A, 407, 741-757, astro-ph/0306329

Dowdy, Jr., J. F., Rabin, D., Moore, R. L., 1986, On the magnetic structure of the quiet transition region, Sol. Phys., 105, 35-45

Dwivedi, B. N., Narain, U., 2008, Physics of the Sun and its Atmosphere 
Ermolli, I., Matthes, K., Dudok de Wit, T., Krivova, N. A., Tourpali, K., Weber, M., Unruh, Y. C., Gray, L., Langematz, U., Pilewskie, P., Rozanov, E., Schmutz, W., Shapiro, A., Solanki, S. K., Thuillier, G., Woods, T. N., 2012, Recent variability of the solar spectral irradiance and its impact on climate modelling, Atmospheric Chemistry and Physics Discussions, 12, 24 557-24 642

Falconer, D. A., Moore, R. L., Porter, J. G., Hathaway, D. H., 2003, Solar Coronal Heating and the Magnetic Flux Content of the Network, ApJ, 593, 549-563

Fawzy, D., Rammacher, W., Ulmschneider, P., Musielak, Z. E., Stępień, K., 2002, Acoustic and magnetic wave heating in stars . I. Theoretical chromospheric models and emerging radiative fluxes, A\&A, 386, 971-982

Feldman, U., 1983, On the unresolved fine structures of the solar atmosphere in the 30,000-200,000 K temperature region, ApJ, 275, 367-373

Feldman, U., 1987, On the unresolved fine structures of the solar atmosphere. II - The temperature region 200,000 - 500,000 K, ApJ, 320, 426-429

Fontenla, J. M., 2005, Chromospheric plasma and the Farley-Buneman instability in solar magnetic regions, A\&A, 442, 1099-1103

Fontenla, J. M., Curdt, W., Avrett, E. H., Harder, J., 2007, Log-normal intensity distribution of the quiet-Sun FUV continuum observed by SUMER, A\&A, 468, 695-699

Fossum, A., Carlsson, M., 2006, Determination of the Acoustic Wave Flux in the Lower Solar Chromosphere, ApJ, 646, 579-592

Fröhlich, C., 2004, Solar Irradiance Variability, in Solar Variability and its Effects on Climate. Geophysical Monograph 141, (Eds.) J. M. Pap, P. Fox, C. Frohlich, H. S. Hudson, J. Kuhn, J. McCormack, G. North, W. Sprigg, S. T. Wu, vol. 141 of Washington DC American Geophysical Union Geophysical Monograph Series, p. 97

Fröhlich, C., 2006, Solar Irradiance Variability Since 1978. Revision of the PMOD Composite during Solar Cycle 21, Space Sci. Rev., 125, 53-65

Fröhlich, C., 2009, Evidence of a long-term trend in total solar irradiance, A\&A, 501, L27-L30

Fröhlich, C., 2011, A four-component proxy model for total solar irradiance calibrated during solar cycles 21-23, Contributions of the Astronomical Observatory Skalnate Pleso, 41, 113-132

Fröhlich, C., 2012, Total Solar Irradiance Observations, Surveys in Geophysics, 33, 453473

Fu, W. Q., 2006, 4 - radiative transfer, in Atmospheric Science (Second Edition), (Eds.) J. M. Wallace, P. V. Hobbs, pp. 113 - 152, Academic Press, San Diego, second edition edn., ISBN 978-0-12-732951-2 
Gabriel, A. H., 1976, A magnetic model of the solar transition region, Royal Society of London Philosophical Transactions Series A, 281, 339-352

Gallagher, P. T., Phillips, K. J. H., Harra-Murnion, L. K., Keenan, F. P., 1998, Properties of the quiet Sun EUV network, A\&A, 335, 733-745

Georgoulis, M. K., Vlahos, L., 1996, Coronal Heating by Nanoflares and the Variability of the Occurence Frequency in Solar Flares, ApJ, 469, L135

Giordano, S., Mancuso, S., 2008, Coronal Rotation at Solar Minimum from UV Observations, ApJ, 688, 656-668

Gontikakis, C., Peter, H., Dara, H. C., 2003, Sizes of quiet Sun transition region structures, A\&A, 408, 743-753

Goodman, M. L., 2000, On the Mechanism of Chromospheric Network Heating and the Condition for Its Onset in the Sun and Other Solar-Type Stars, ApJ, 533, 501-522

Griffiths, N. W., Fisher, G. H., Woods, D. T., Siegmund, O. H. W., 1999, SUMER Observations of the Quiet-Sun Transition Region, ApJ, 512, 992-1005

Grönholm, T., Annila, A., 2007, Natural distribution, Mathematical Biosciences, 210, 659 - 667, ISSN 0025-5564

Hale, G. E., Ellerman, F., Nicholson, S. B., Joy, A. H., 1919, The Magnetic Polarity of Sun-Spots, ApJ, 49, 153

HamamatsuPhotonics., 2007, Photomultiplier Tubes: Basics and Applications, Hamamatsu Photonics K.K., 3rd edn.

Harrison, R. A., Sawyer, E. C., Carter, M. K., Cruise, A. M., Cutler, R. M., Fludra, A., Hayes, R. W., Kent, B. J., Lang, J., Parker, D. J., Payne, J., Pike, C. D., Peskett, S. C., Richards, A. G., Gulhane, J. L., Norman, K., Breeveld, A. A., Breeveld, E. R., Al Janabi, K. F., McCalden, A. J., Parkinson, J. H., Self, D. G., Thomas, P. D., Poland, A. I., Thomas, R. J., Thompson, W. T., Kjeldseth-Moe, O., Brekke, P., Karud, J., Maltby, P., Aschenbach, B., Bräuninger, H., Kühne, M., Hollandt, J., Siegmund, O. H. W., Huber, M. C. E., Gabriel, A. H., Mason, H. E., Bromage, B. J. I., 1995, The Coronal Diagnostic Spectrometer for the Solar and Heliospheric Observatory, Sol. Phys., 162, 233-290

Harvey, K. L., Zwaan, C., 1993, Properties and emergence of bipolar active regions, Sol. Phys., 148, 85-118

Hathaway, D. H., Upton, L., 2014, The solar meridional circulation and sunspot cycle variability, Journal of Geophysical Research (Space Physics), 119, 3316-3324, 1404. 5893

Henoux, J. C., Somov, B. V., 1991, The photospheric dynamo. I - Magnetic flux-tube generation, A\&A, 241, 613-617

Henoux, J. C., Somov, B. V., 1997, The photospheric dynamo. I. Physics of thin magnetic flux tubes., A\&A, 318, 947-956 
Hudson, H. S., 1991, Solar flares, microflares, nanoflares, and coronal heating, Sol. Phys., $133,357-369$

Jafarzadeh, S., 2013, Dynamics of magnetic bright points in the lower solar atmosphere, Ph.D. thesis, Institut für Astrophysik, Georg-August-Universität Göttingen

Jiang, J., Chatterjee, P., Choudhuri, A. R., 2007, Solar activity forecast with a dynamo model, MNRAS, 381, 1527-1542, 0707.2258

Jin, C., Wang, J., Zhao, M., 2009, Vector Magnetic Fields of Solar Granulation, ApJ, 690, 279-287, 0809.0956

Judge, D. L., McMullin, D. R., Ogawa, H. S., Hovestadt, D., Klecker, B., Hilchenbach, M., Mobius, E., Canfield, L. R., Vest, R. E., Watts, R., Tarrio, C., Kuehne, M., Wurz, P., 1998, First Solar EUV Irradiances Obtained from SOHO by the CELIAS/SEM, Sol. Phys., 177, 161-173

Kalkofen, W., 2007, Is the Solar Chromosphere Heated by Acoustic Waves?, ApJ, 671, 2154-2158

Klimchuk, J. A., 2006, On Solving the Coronal Heating Problem, Sol. Phys., 234, 41-77, astro-ph/0511841

Klimchuk, J. A., 2009, Coronal Loop Models and Those Annoying Observations! (Keynote), in The Second Hinode Science Meeting: Beyond Discovery-Toward Understanding, (Eds.) B. Lites, M. Cheung, T. Magara, J. Mariska, K. Reeves, vol. 415 of Astronomical Society of the Pacific Conference Series, p. 221, 0904 .1391

Knaack, R., Stenflo, J. O., Berdyugina, S. V., 2005, Evolution and rotation of large-scale photospheric magnetic fields of the Sun during cycles 21-23. Periodicities, north-south asymmetries and r-mode signatures, A\&A, 438, 1067-1082

Kontar, E. P., Hannah, I. G., MacKinnon, A. L., 2008, Chromospheric magnetic field and density structure measurements using hard X-rays in a flaring coronal loop, A\&A, 489, L57-L60, 0808. 3334

Kundu, M. R., 1985, High spatial resolution microwave observations of the sun, Sol. Phys., $100,491-514$

Kuperus, M., Ionson, J. A., Spicer, D. S., 1981, On the theory of coronal heating mechanisms, ARA\&A, 19, 7-40

Ledrew, G., 2001, The Real Starry Sky, JRASC, 95, 32

Leighton, R. B., 1964, Transport of Magnetic Fields on the Sun., ApJ, 140, 1547

Leighton, R. B., Noyes, R. W., Simon, G. W., 1962, Velocity Fields in the Solar Atmosphere. I. Preliminary Report., ApJ, 135, 474

Levine, R. H., 1974, Acceleration of Thermal Particles in Collapsing Magnetic Regions, ApJ, 190, 447-456 
Lighthill, M. J., 1952, On sound generated aerodynamically. i. general theory, Proceedings of the Royal Society of London. Series A. Mathematical and Physical Sciences, 211, 564-587, http://rspa.royalsocietypublishing.org/content/ 211/1107/564. full.pdf+html

Limpert, E., Stahel, W. A., Abbt, M., 2001, Log-normal distributions across the sciences: Keys and clues, BioScience, 51, 341-352

Lites, B. W., Martinez Pillet, V., Skumanich, A., 1994, A quantitative comparison of vector magnetic field measurement and analysis techniques, Sol. Phys., 155, 1-27

Lites, B. W., Rutten, R. J., Berger, T. E., 1999, Dynamics of the Solar Chromosphere. II. Ca II $\mathrm{H}_{2 V}$ and $\mathrm{K}_{2 V}$ Grains versus Internetwork Fields, ApJ, 517, 1013-1033

Lites, B. W., Kubo, M., Socas-Navarro, H., Berger, T., Frank, Z., Shine, R., Tarbell, T., Title, A., Ichimoto, K., Katsukawa, Y., Tsuneta, S., Suematsu, Y., Shimizu, T., Nagata, S., 2008, The Horizontal Magnetic Flux of the Quiet-Sun Internetwork as Observed with the Hinode Spectro-Polarimeter, ApJ, 672, 1237-1253

Livingston, W., White, O. R., Wallace, L., Harvey, J., 2010, Sun-as-a-Star, Chromospheric Lines, 1974-2009, Mem. Soc. Astron. Italiana, 81, 643

Lockwood, M., Owens, M., Rouillard, A. P., 2009, Excess open solar magnetic flux from satellite data: 2. A survey of kinematic effects, Journal of Geophysical Research (Space Physics), 114, A11104

Lockwood, M., Harrison, R. G., Woollings, T., Solanki, S. K., 2010, Are cold winters in Europe associated with low solar activity?, Environmental Research Letters, 5, 024001

Loukitcheva, M., Solanki, S. K., White, S. M., 2009, The relationship between chromospheric emissions and magnetic field strength, A\&A, 497, 273-285, 0910. 1985

Lukianova, R., 2009, Discrepancy in behavior of different solar proxies in cycle 23, in EGU General Assembly Conference Abstracts, (Eds.) D. N. Arabelos, C. C. Tscherning, vol. 11 of EGU General Assembly Conference Abstracts, p. 11903

Malinovsky, M., 1975, New calculations of atomic data concerning E.U.V. lines of O V, A\&A, 43, 101-110

Martínez Pillet, V., 2013, Solar Surface and Atmospheric Dynamics. The Photosphere, Space Sci. Rev., 178, 141-162, 1301.6933

Maunder, E. W., 1903, Spoerer's law of zones, The Observatory, 26, 329-330

Mekaoui, S., Dewitte, S., 2008, Total Solar Irradiance Measurement and Modelling during Cycle 23, Sol. Phys., 247, 203-216

Miesch, M. S., 2005, Large-Scale Dynamics of the Convection Zone and Tachocline, Living Reviews in Solar Physics, 2, 1 
Nandy, D., 2012, Modeling the solar cycle: what the future holds, in IAU Symposium, (Eds.) C. H. Mandrini, D. F. Webb, vol. 286 of IAU Symposium, pp. 54-64, 1111.5352

Narain, U., Pandey, K., 2006, Nanoflares and Heating of the Solar Corona, Journal of Astrophysics and Astronomy, 27, 93-100

Narain, U., Ulmschneider, P., 1996, Chromospheric and Coronal Heating Mechanisms II, Space Sci. Rev., 75, 453-509

Nindos, A., Zirin, H., 1998, The Relation of CA II K Features to Magnetic Field, Sol. Phys., $179,253-268$

Ossendrijver, M., 2003, The solar dynamo, A\&A Rev., 11, 287-367

Parker, E. N., 1955, Hydromagnetic Dynamo Models., ApJ, 122, 293

Parker, E. N., 1972, Topological Dissipation and the Small-Scale Fields in Turbulent Gases, ApJ, 174, 499

Parker, E. N., 1975, The generation of magnetic fields in astrophysical bodies. X - Magnetic buoyancy and the solar dynamo, ApJ, 198, 205-209

Parker, E. N., 1988, Nanoflares and the solar X-ray corona, ApJ, 330, 474-479

Parnell, C. E., De Moortel, I., 2012, A contemporary view of coronal heating, Royal Society of London Philosophical Transactions Series A, 370, 3217-3240, 1206.6097

Patsourakos, S., Vial, J.-C., Gabriel, A. H., Bellamine, N., 1999, Transition-Region Network Boundaries in the Quiet Sun: Width Variation with Temperature as Observed with CDS on SOHO, ApJ, 522, 540-546

Pauluhn, A., Solanki, S. K., 2007, A nanoflare model of quiet Sun EUV emission, A\&A, 462, 311-322, arXiv:astro-ph/0612585

Pauluhn, A., Rüedi, I., Solanki, S. K., Lang, J., Pike, C. D., Schühle, U., Thompson, W. T., Hollandt, J., Huber, M. C. E., 1999, Intercalibration of sumer and cds on soho. i. sumer detector a and cds nis, Appl. Opt., 38, 7035-7046

Pauluhn, A., Solanki, S. K., Rüedi, I., Landi, E., Schühle, U., 2000, Statistics of quiet Sun extreme ultraviolet intensities, A\&A, 362, 737-745

Pauluhn, A., Rüedi, I., Solanki, S. K., Schühle, U., Wilhelm, K., Lang, J., Thompson, W. T., Hollandt, J., 2001, Intercalibration of SUMER and CDS on SOHO. II. SUMER detectors A and B and CDS NIS, Appl. Opt., 40, 6292-6300

Petrovay, K., 2010, Solar Cycle Prediction, Living Reviews in Solar Physics, 7, 6, 1012. 5513

Phillips, K. J. H., 1995, Guide to the Sun, Cambridge University Press

Pike, C. D., Harrison, R. A., 2000, Long-duration cosmic ray modulation from a Sun-Earth L1 orbit, A\&A, 362, L21-L24 
Potgieter, M., 2013, Solar Modulation of Cosmic Rays, Living Reviews in Solar Physics, $10,3,1306.4421$

Press, W. H., Flannery, B. P., Teukolsky, S. A., Vetterling, W. T., 1988, Numerical Recipes in C: The Art of Scientific Computing, Cambridge University Press, New York, NY, USA, ISBN 0-521-35465-X

Priest, E., Forbes, T. (Eds.), 2000, Magnetic reconnection : MHD theory and applications

Rabin, D., Moore, R., 1984, Heating the sun's lower transition region with fine-scale electric currents, ApJ, 285, 359-367

Ravindra, B., Venkatakrishnan, P., 2003, On the Correlation Between the he ii 1304 Network Brightening and the Photospheric Magnetic Field, Sol. Phys., 214, 267-285

Reale, F., 2010, Coronal Loops: Observations and Modeling of Confined Plasma, Living Reviews in Solar Physics, 7, 5, 1010. 5927

Reeves, E. M., 1976, The EUV chromospheric network in the quiet sun, Sol. Phys., 46, $53-72$

Remling, B., Deubner, F.-L., Steffens, S., 1996, Evidence of K_2v_grains being a nonmagnetic phenomenon., A\&A, 316, 196-200

Rempel, M., 2014, Numerical Simulations of Quiet Sun Magnetism: On the Contribution from a Small-scale Dynamo, ApJ, 789, 132, 1405.6814

Rezaei, R., Schlichenmaier, R., Beck, C. A. R., Bruls, J. H. M. J., Schmidt, W., 2007, Relation between photospheric magnetic field and chromospheric emission, A\&A, 466, 1131-1144, arXiv: astro-ph/0701896

Roberts, B., 2000, Waves and Oscillations in the Corona - (Invited Review), Sol. Phys., 193, 139-152

Rutten, R. J., Uitenbroek, H., 1991, CA II H(2v) and K(2v) cell grains, Sol. Phys., 134, $15-71$

Samain, D., Bonnet, R. M., Gayet, R., Lizambert, C., 1975, Stigmatic spectra of the sun between $1200 \mathrm{~A}$ and $2100 \mathrm{~A}, \mathrm{~A} \& \mathrm{~A}, 39,71-81$

Sánchez Almeida, J., 2003, Inter-Network magnetic fields observed during the minimum of the solar cycle, A\&A, 411, 615-621, astro-ph/0310164

Sánchez Almeida, J., Martínez González, M., 2011, The Magnetic Fields of the Quiet Sun, in Solar Polarization 6, (Eds.) J. R. Kuhn, D. M. Harrington, H. Lin, S. V. Berdyugina, J. Trujillo-Bueno, S. L. Keil, T. Rimmele, vol. 437 of Astronomical Society of the Pacific Conference Series, p. 451, 1105.0387

Scafetta, N., Willson, R. C., 2009, ACRIM-gap and TSI trend issue resolved using a surface magnetic flux TSI proxy model, Geophys. Res. Lett., 36, L05701 
Scherrer, P. H., Bogart, R. S., Bush, R. I., Hoeksema, J. T., Kosovichev, A. G., Schou, J., Rosenberg, W., Springer, L., Tarbell, T. D., Title, A., Wolfson, C. J., Zayer, I., MDI Engineering Team, 1995, The Solar Oscillations Investigation - Michelson Doppler Imager, Sol. Phys., 162, 129-188

Schou, J., Christensen-Dalsgaard, J., Thompson, M. J., 1992, The resolving power of current helioseismic inversions for the sun's internal rotation, ApJ, 385, L59-L62

Schrijver, C. J., 1987, Magnetic structure in cool stars. XI - Relations between radiative fluxes measuring stellar activity, and evidence for two components in stellar chromospheres, A\&A, 172, 111-123

Schrijver, C. J., 1995, Basal heating in the atmospheres of cool stars, A\&A Rev., 6, $181-223$

Schrijver, C. J., Liu, Y., 2008, The Global Solar Magnetic Field Through a Full Sunspot Cycle: Observations and Model Results, Sol. Phys., 252, 19-31

Schrijver, C. J., Siscoe, G. L., 2010, Heliophysics: Evolving Solar Activity and the Climates of Space and Earth, Cambridge University Press

Schrijver, C. J., Siscoe, G. L., 2012, Heliophysics: Evolving Solar Activity and the Climates of Space and Earth

Schrijver, C. J., Zwaan, C., 2000, Solar and Stellar Magnetic Activity

Schrijver, C. J., Cote, J., Zwaan, C., Saar, S. H., 1989, Relations between the photospheric magnetic field and the emission from the outer atmospheres of cool stars. I - The solar CA II K line core emission, ApJ, 337, 964-976

Schrijver, C. J., Livingston, W. C., Woods, T. N., Mewaldt, R. A., 2011, The minimal solar activity in 2008-2009 and its implications for long-term climate modeling, Geophys. Res. Lett., 38, L06701

Schröder, K.-P., Mittag, M., Pérez Martínez, M. I., Cuntz, M., Schmitt, J. H. M. M., 2012, Basal chromospheric flux and Maunder Minimum-type stars: the quiet-Sun chromosphere as a universal phenomenon, A\&A, 540, A130, 1202.3314

Schüssler, M., 1979, Magnetic buoyancy revisited - Analytical and numerical results for rising flux tubes, A\&A, 71, 79-91

Schüssler, M., 1986, MHD Models of Solar Photospheric Magnetic Flux Concentrations, in Small Scale Magnetic Flux Concentrations in the Solar Photosphere, (Eds.) W. Deinzer, M. Knölker, H. H. Voigt, p. 103

Schüssler, M., 1990, Theoretical Aspects of Small-Scale Photospheric Magnetic Fields, in Solar Photosphere: Structure, Convection, and Magnetic Fields, (Ed.) J. O. Stenflo, vol. 138 of IAU Symposium, p. 161

Schüssler, M., Vögler, A., 2008, Strong horizontal photospheric magnetic field in a surface dynamo simulation, A\&A, 481, L5-L8, 0801.1250 
Schwarzschild, M., 1948, On Noise Arising from the Solar Granulation., ApJ, 107, 1

Scott, D. W., 1979, On optimal and data-based histograms, Biometrika, 66, 605-610, http://biomet. oxfordjournals .org/content/66/3/605. full.pdf+html

Seely, J. F., Feldman, U., 1985, Population mechanisms for the $\mathrm{He}(+) \mathrm{N}=3$ levels determined from measurements of the solar 1640 A emission, MNRAS, 213, 417-434

Shakeri, F., Teriaca, L., Solanki, S. K., 2015, Solar extreme ultraviolet variability of the quiet Sun, A\&A, 581, A51, 1507.05786

Simon, G. W., Leighton, R. B., 1964, Velocity Fields in the Solar Atmosphere. III. LargeScale Motions, the Chromospheric Network, and Magnetic Fields., ApJ, 140, 1120

Sivaraman, K. R., Gupta, S. S., Livingston, W. C., Damé, L., Kalkofen, W., Keller, C. U., Smartt, R., Hasan, S. S., 2000, Results from a revisit to the $\mathrm{K}_{2 V}$ bright points, A\&A, 363, 279-288

Skumanich, A., Smythe, C., Frazier, E. N., 1975, On the statistical description of inhomogeneities in the quiet solar atmosphere. I - Linear regression analysis and absolute calibration of multichannel observations of the $\mathrm{Ca} /+$ / emission network, ApJ, 200, 747-764

Solanki, S. K., 1996, Solar and stellar magnetic flux tubes (review), in Stellar Surface Structure, (Eds.) K. G. Strassmeier, J. L. Linsky, vol. 176 of IAU Symposium, p. 201

Solanki, S. K., Krivova, N. A., 2011, Analyzing Solar Cycles, Science, 334, 916-

Solanki, S. K., Unruh, Y. C., 1998, A model of the wavelength dependence of solar irradiance variations, A\&A, 329, 747-753

Solanki, S. K., Inhester, B., Schüssler, M., 2006, The solar magnetic field, Reports on Progress in Physics, 69, 563-668, 1008.0771

Solanki, S. K., Krivova, N. A., Haigh, J. D., 2013, Solar Irradiance Variability and Climate, ARA\&A, 51, 311-351, 1306.2770

Solomon, S. C., Woods, T. N., Didkovsky, L. V., Emmert, J. T., Qian, L., 2010, Anomalously low solar extreme-ultraviolet irradiance and thermospheric density during solar minimum, Geophys. Res. Lett., 37, L16103

Spruit, H. C., 1976, Pressure equilibrium and energy balance of small photospheric fluxtubes, Sol. Phys., 50, 269-295

Spruit, H. C., 1977, Magnetic flux tubes and transport of heat in the convection zone of the Sun., Ph.D. thesis, Thesis University of Utrecht, The Netherlands.

Spruit, H. C., Title, A. M., van Ballegooijen, A. A., 1987, Is there a weak mixed polarity background field? Theoretical arguments, Sol. Phys., 110, 115-128

Stein, R. F., 1967, Generation of Acoustic and Gravity Waves by Turbulence in an Isothermal Stratified Atmosphere, Sol. Phys., 2, 385-432 
Stein, R. F., 1968, Waves in the Solar Atmosphere. I. The Acoustic Energy Flux., ApJ, 154,297

Stein, R. F., Nordlund, A., 2002, Solar Surface Magneto-Convection and Dynamo Action, in SOLMAG 2002. Proceedings of the Magnetic Coupling of the Solar Atmosphere Euroconference, (Ed.) H. Sawaya-Lacoste, vol. 505 of ESA Special Publication, pp. 83-89

Stenflo, J. O., 2012, Basal magnetic flux and the local solar dynamo, A\&A, 547, A93, 1210.0122

Svalgaard, L., Hudson, H. S., 2010, The Solar Microwave Flux and the Sunspot Number, in SOHO-23: Understanding a Peculiar Solar Minimum, (Eds.) S. R. Cranmer, J. T. Hoeksema, J. L. Kohl, vol. 428 of Astronomical Society of the Pacific Conference Series, p. $325,1003.4281$

Tajfirouze, E., Safari, H., 2012, Can a Nanoflare Model of Extreme-ultraviolet Irradiances Describe the Heating of the Solar Corona?, ApJ, 744, 113, 1112.4926

Tapping, K. F., Valdés, J. J., 2011, Did the Sun Change Its Behaviour During the Decline of Cycle 23 and Into Cycle 24?, Sol. Phys., 272, 337-350

Testa, P., De Pontieu, B., Martínez-Sykora, J., DeLuca, E., Hansteen, V., Cirtain, J., Winebarger, A., Golub, L., Kobayashi, K., Korreck, K., Kuzin, S., Walsh, R., DeForest, C., Title, A., Weber, M., 2013, Observing Coronal Nanoflares in Active Region Moss, ApJ, 770, L1, 1305. 1687

Thompson, W., Carter, M., 1998, EUV Full-Sun Imaging and Pointing Calibration of the SOHO Coronal Diagnostic Spectrometer, Sol. Phys., 178, 71-83

Thompson, W. T., 2000, Differential scrubbing in a microchannel-plate intensified CCD detector, Optical Engineering, 39, 2651-2659

Thompson, W. T., Poland, A. I., Siegmund, O. H., Swartz, M., Leviton, D. B., Payne, L. J., 1992, Measurements of an intensified CCD detector for the Solar and Heliospheric Observatory, in EUV, X-Ray, and Gamma-Ray Instrumentation for Astronomy III, (Ed.) O. H. W. Siegmund, vol. 1743 of Society of Photo-Optical Instrumentation Engineers (SPIE) Conference Series, pp. 464-474

Thornton, L. M., Parnell, C. E., 2011, Small-Scale Flux Emergence Observed Using Hinode/SOT, Sol. Phys., 269, 13-40

Title, A. M., Schrijver, C. J., 1998, The Sun's Magnetic Carpet, in Cool Stars, Stellar Systems, and the Sun, (Eds.) R. A. Donahue, J. A. Bookbinder, vol. 154 of Astronomical Society of the Pacific Conference Series, p. 345

Tripathy, S. C., Jain, K., Hill, F., Leibacher, J. W., 2010, Unusual Trends in Solar P-Mode Frequencies During the Current Extended Minimum, ApJ, 711, L84-L88, 1002 . 1690

Trujillo Bueno, J., Shchukina, N., Asensio Ramos, A., 2004, A substantial amount of hidden magnetic energy in the quiet Sun, Nature, 430, 326-329, astro-ph/0409004 
Ulmschneider, P., 1974, Radiation loss and mechanical heating in the solar chromosphere, Sol. Phys., 39, 327-336

Ulmschneider, P., Kalkofen, W., 2003, Heating of the solar chromosphere, pp. 181-195

Upton, L., Hathaway, D. H., 2014, Effects of Meridional Flow Variations on Solar Cycles 23 and 24, ArXiv e-prints, 1408.0035

Usoskin, I. G., 2013, A History of Solar Activity over Millennia, Living Reviews in Solar Physics, 10, 1

Vernazza, J. E., Avrett, E. H., Loeser, R., 1981, Structure of the solar chromosphere. III Models of the EUV brightness components of the quiet-sun, ApJS, 45, 635-725

Vieira, L. E. A., Solanki, S. K., 2010, Evolution of the solar magnetic flux on time scales of years to millenia, A\&A, 509, A100, 0911.4396

Vögler, A., Schüssler, M., 2007, A solar surface dynamo, A\&A, 465, L43-L46, arXiv: astro-ph/0702681

Walsh, R. W., Ireland, J., 2003, The heating of the solar corona, A\&A Rev., 12, 1-41

Wang, Y.-M., Robbrecht, E., Sheeley, Jr., N. R., 2009, On the Weakening of the Polar Magnetic Fields during Solar Cycle 23, ApJ, 707, 1372

Weber, M., Pagaran, J., Dikty, S., Savigny, C., Burrows, J., DeLand, M., Floyd, L., Harder, J., Mlynczak, M., Schmidt, H., 2013, Investigation of solar irradiance variations and their impact on middle atmospheric ozone, in Climate and Weather of the Sun-Earth System (CAWSES), (Ed.) F.-J. Lübken, Springer Atmospheric Sciences, pp. 39-54, Springer Netherlands, ISBN 978-94-007-4347-2

Wedemeyer-Böhm, S., Wöger, F., 2008, Small-scale structure and dynamics of the lower solar atmosphere, in IAU Symposium, (Eds.) R. Erdélyi, C. A. Mendoza-Briceno, vol. 247 of IAU Symposium, pp. 66-73, 0710.4776

Wedemeyer-Böhm, S., Steiner, O., Bruls, J., Rammacher, W., 2007, What is Heating the Quiet-Sun Chromosphere?, in The Physics of Chromospheric Plasmas, (Eds.) P. Heinzel, I. Dorotovič, R. J. Rutten, vol. 368 of Astronomical Society of the Pacific Conference Series, p. 93, astro-ph/0612627

Wedemeyer-Böhm, S., Lagg, A., Nordlund, Å., 2009, Coupling from the Photosphere to the Chromosphere and the Corona, Space Sci. Rev., 144, 317-350, 0809.0987

Wiegelmann, T., Thalmann, J. K., Solanki, S. K., 2014, The Magnetic Field in the Solar Atmosphere, ArXiv e-prints, 1410.4214

Wilhelm, K., Woods, T. N., Schühle, U., Curdt, W., Lemaire, P., Rottman, G. J., 1999, The solar ultraviolet spectrum from $1200 \AA$ to $1560 \AA$ : a radiometric comparison between SUMER/SOHO and SOLSTICE/UARS, A\&A, 352, 321-326 
Wilhelm, K., Dwivedi, B. N., Marsch, E., Feldman, U., 2004, Observations of the Sun at Vacuum- Ultraviolet Wavelengths from Space. Part I: Concepts and Instrumentation, Space Sci. Rev., 111, 415-480

Wilhelm, K., Marsch, E., Dwivedi, B. N., Feldman, U., 2007, Observations of the Sun at Vacuum-Ultraviolet Wavelengths from Space. Part II: Results and Interpretations, Space Sci. Rev., 133, 103-179

Willson, R. C., Mordvinov, A. V., 2003, Secular total solar irradiance trend during solar cycles 21-23, Geophys. Res. Lett., 30, 1199

Woods, T., 2002, Solar vacuum ultraviolet irradiance measurements and models for solar cycle 23, in From Solar Min to Max: Half a Solar Cycle with SOHO, (Ed.) A. Wilson, vol. 508 of ESA Special Publication, pp. 165-172

Woods, T. N., 2010, Irradiance Variations during This Solar Cycle Minimum, in SOHO23: Understanding a Peculiar Solar Minimum, (Eds.) S. R. Cranmer, J. T. Hoeksema, J. L. Kohl, vol. 428 of Astronomical Society of the Pacific Conference Series, p. 63, 1003.4524

Woods, T. N., Rottman, G. J., 2002, Solar Ultraviolet Variability Over Time Periods of Aeronomic Interest, Washington DC American Geophysical Union Geophysical Monograph Series, 130, 221

Yeo, K. L., Feller, A., Solanki, S. K., Couvidat, S., Danilovic, S., Krivova, N. A., 2014, Point spread function of SDO/HMI and the effects of stray light correction on the apparent properties of solar surface phenomena, A\&A, 561, A22, 1310.4972

Yung, Y. L., 2003, An introduction to atmospheric radiation. by k. n. liou. academic press. second edition, 2002. pp. xiv+583. isbn 012451451 0, Quarterly Journal of the Royal Meteorological Society, 129, 1741-1741, ISSN 1477-870X

Zirin, H., 1988, Book-Review - Astrophysics of the Sun, Journal of the British Astronomical Association, 98, 316 



\section{Acknowledgements}

This $\mathrm{PhD}$ thesis is the result of my research work during the last few years, at the Max Planck Institute for Solar System Research (MPS). There are many people who have directly and indirectly contributed in my research, without whom this work would not be possible.

First and foremost, I wish to thank Dr. Luca Teriaca, at Max Planck Institute for Solar System Research (MPS), for his tremendous support during my PhD. I deeply feel indebted to him for his daily supervision and for helping me to crank out this dissertation. I would also like to express my special appreciation and thanks to Prof. Dr. Sami K. Solanki, my supervisor at MPS, for his priceless advice and remarkably constructive feedback. Reviewing a thesis is no easy task, I am very grateful to them both for their insightful and extremely detailed comments. I would also like to thank Prof. Dr. Stefan Dreizler for accepting to be my supervisor at the University of Göttingen, and for his valuable suggestions about my research.

It has been my privilege to be a member of the International Max Planck Research School (IMPRS). I acknowledge MPS for funding my thesis by granting me the PhD fellowship. I exclusively would like to thank the late Dr. Dieter Schmitt, the ex-coordinator of the IMPRS. He deserves my heartfelt gratitude for all moral and emotional support he dedicated to me, specially at the beginning of my PhD study. I also wish to thank Dr. Sonja Schuh, the current coordinator of the MPS research school for her efficient supervision of the IMPRS.

I will forever be thankful to all my former university professors. Special thanks go to Profs. Mohammad Sadegh Alaee and Shahriar Bayegan at the University of Tehran, and Prof. Dr. Christian Von Savigny, Dr. Marc Weber and Dr. Helmut Fischer at the University of Bremen. They all contribute in the completion of my studies in different ways, and I was very fortunate for being their student.

There are dozens of friends whom I am indebted to, for their valuable friendship, support and care during the last few years: Juanjo, Karsten, Chemeda, Tijmen, Wieland, Ines, Philippe, Julia, Shahin, David, Maria, Kok Leng, Nafiseh, Neda, Vali, Fatima, Sebastian, Borys, Ricardo, Neil, Francisco, David, Anna, Martin, Donald, Atefeh, Ankit, Chaytania, Jishesh, and many more whom I undoubtedly forgot. I wish to thank my Iranian friends who made here like home for me, and with whom I have numerous amazing and wonderful memories: Ali, Hanieh, Sahrokh, Layla, Behrooz, Elham, Nazli, Reza, Lena-che, Rayan, Hooman, Elmira, Sina, Dena, Emad, Tara, Aydin, Alireza and Zahra, thank you for providing support and friendship which I needed.

This thesis was done in the memory of all the love ones I lost during these years: uncle Jalal, cousine Behzad, aunt Saeedeh, uncle Jamal, and Dr. Rahim Ashari.

And finally, the source of my life energy, my personal sun, my family. There are no 
words to convey how much I love you. Shabnam, my beautiful wife, the one who was always sticking by my side, during my best and my worst. Thank you for having faith in me, even when I felt empty inside and lost my faith in myself. Thank you for your endless love, for your shining smiles. My parents, who are the reason, who are at the infinity of my love, who taught me the meaning of life, who showed me how not to give up. Thank you for everything you gave me, for guiding me through the maze of my life. My brother, from whom I learnt, it doesn't matter how others want you to be, but how you yourself want to be. Thank you for teaching me that dreaming is as important as thinking. And again to my brother and his wonderful wife, Sepideh, thank you for filling up our paternal house with your warm presence, and for not letting my parents feel lonely. To all of you ...

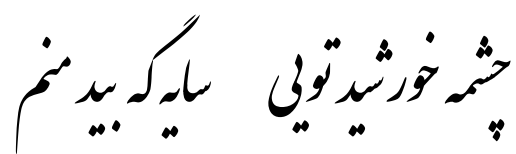

"You, the fountain of the Sun's light.

$\mathrm{Me}$, a willow shadow on the ground." $\sim$ Rumi 


\section{Curriculum Vitae}

\section{Farhad Shakeri}

Born in April 1983 in Tehran, Iran

Married, Iranian

E-Mail: farhad.shakeri@gmail.com

\section{Education}

09/2001-07/2007 Bachelor of Science in Physics University of Tehran

10/2008-06/2010 Master of Science in Environmental Physics University of Bremen, Institute for Environmental Physics

11/2010-01/2015 Ph.D in Physics

University of Göttingen and Max Planck Institute for Soar System

\section{Publications and Conference contributions}

Solar extreme ultraviolet variability of the quiet Sun

F. Shakeri, L. Teriaca, S. K. Solanki, A\&A, 2015, arXiv:1507.05786

Natonal Astronomy Meeting, July 5-9, 2015, Llandudno, Wales

Solar VUV variability of the quiet Sun (Talk)

F. Shakeri, L. Teriaca, S. K. Solanki

Space Climate 5 Symposium, June 15-19, 2013, Oulu, Finland

Solar VUV variability of the quiet Sun (Poster)

F. Shakeri, L. Teriaca, S. K. Solanki

AGU Chapman Conference on Causes and Consequences of the Extended Solar Minimum Between Solar Cycles 23 and 24 (4CESM), April 08-12, 2013, Key Largo, U.S.A Cycle-related solar VUV variability (Talk accepted, but cancelled due to visa issues) F. Shakeri, L. Teriaca, S. K. Solanki

Rocks'n'Stars Conference, October 8-11, 2012, Göttingen, Germany Cycle-related solar Vacuum Ultraviolet variability (Poster)

F. Shakeri, L. Teriaca, S. K. Solanki

October 19, 2015 